\title{
Brigitte Fleury
}

Maîtrise en communication, Université du Québec à Montréal

(2004)

\section{Étude de la conversion religieuse d'un point de vue communicationnel: le cas de Roger Garaudy}

Un document produit en version numérique par Jean-Marie Tremblay, bénévole, professeur de sociologie au Cégep de Chicoutimi

Courriel: jmt_sociologue@videotron.ca

Site web: http://pages.infinit.net/sociojmt

Dans le cadre de la collection: "Les classiques des sciences sociales"

Site web: http://www.uqac.uquebec.ca/zone30/Classiques_des_sciences_sociales/index.html

Une collection développée en collaboration avec la Bibliothèque

Paul-Émile-Boulet de l'Université du Québec à Chicoutimi

Site web: http://bibliotheque.uqac.uquebec.ca/index.htm 
Cette édition électronique a été réalisée par Jean-Marie Tremblay, bénévole, professeur de sociologie au Cégep de Chicoutimi à partir du mémoire de maitrise en communication de :

Brigitte Fleury, Étude de la conversion religieuse d'un point de vue communicationnel: le cas de Roger Garaudy. Montréal : mémoire de maître présenté au département des communications, juin 2004.

Thèse fortement recommandée par son tuteur, M. René-Jean Ravault, sociologue, UQAM et préfacé par M. Ravault.

Autorisation accordée par l'auteure le 15 juillet 2004 de diffuser ce mémoire.

됴 Courriel : BFleury001@aol.com

Polices de caractères utilisée :

Pour le texte: Times, 12 points.

Pour les citations : Times 10 points.

Pour les notes de bas de page : Times, 10 points.

Édition électronique réalisée avec le traitement de textes Microsoft Word 2001 pour Macintosh.

Mise en page sur papier format

LETTRE (US letter), 8.5' x 11'')

Édition numérique réalisée le 7 janvier 2005 à Chicoutimi, Ville de Saguenay, province de Québec, Canada. 


\title{
Table des matières
}

\author{
$\underline{\text { Remerciements }}$
}

Préface de René-Jean Raveault, 31 août 2004

Résumé

Introduction

Chapitre I : Développement de la problématique

1-1 Lacunes des analyses du phénomène de conversion religieuse

1-2 Le choix de l'approche constructiviste

1-3 Problématique

1-3.1 Question et hypothèse centrales

1-3.2 Hypothèses sectorielles

Chapitre II : Cadre de référence théorique

2-1 Aperçu de la recherche sur les conversions à l'islam dans le contexte européen

2-2 Distribution sociale de la connaissance

2-3 $\quad$ Sur le sens du processus de réception

2-3.1 La socialisation primaire

2-3.2 Formation de l'identité par «coerséduction»

2-3.3 Ouverture au changement d'affiliation sur le plan religieux

2-3.4 La communication intrapersonnelle

2-3.5 La communication interpersonnelle

2-3.6 Cognition et variations de l'interprétation

2-3.6.1 La mémoire

2-3.6.2 L'imagination

2-3.6.3 Perception renouvelée et intuition

2-3.6.4 Le jugement 


\section{2-4 Appréhension du contexte social}

2-4.1 Contexte social et visions du monde

2-4.2 Pratique renouvelée de l'acte réflexif

\section{2-4.2.1 En guise d'exemple}

2-4.3 Interprétation du changement contextuel dans les pratiques culturelles

2-5 Construction des univers communicationnels dans les relations de groupe

2-5.1 Types d'entreprises sociales relatives au cas de conversion

2-5.2 Particularisme et universalisme des réalités communicationnelles

2-5.3 Conscience des accords tacites et élaboration des univers communicationnels

2-6 Conversion des univers communicationnels reliée aux conflits d'interprétation

2-6.1 Crise interprétative relative à l'éthique

\section{2-7 Conclusion}

Chapitre III : Étude de cas : la conversion de Roger Garaudy

3-1 Biographie sommaire de Roger Garaudy

3-2 Étude de la conversion de Roger Garaudy

3-2.1 Fondements de la vision du monde de Garaudy

3-2.1.1 À propos des concepts de transcendance et d'immanence

3-2.1.2 Construction de la problématique philosophique de Roger Garaudy

3-2.1.3 Deux visions opposées du monde

3-2.2 Détachement identitaire et conflits interpersonnels

3-2.2.1 La conversion de la Malinche

3-2.2.2 Décodage et divergences d'interprétation

3-2.2.3 Du catholicisme

3-2.2.4 Du marxisme

3-3 Résolution des conflits d'interprétation par la conversion 
Conclusion

Bibliographie 


\title{
Préface
}

\author{
au mémoire de maîtrise de Brigitte Fleury: \\ "Étude sur la construction de l'expérience de conversion religieuse \\ d'un point de vue communicationnel, \\ le cas de Roger Garaudy "
}

Par René-Jean Ravault, Sociologue, professeur au département de communications, UQAM

Montréal, le 31 août 2004

\section{$\underline{\text { Retour à la table des matières }}$}

Rédiger la préface d'un ouvrage que l'on n'a pas soi-même écrit est une tâche délicate. Tout en s'efforçant de renforcer l'estime qui nous lie à l'auteur en démontrant que l'on a bien saisi l'essentiel de ce qu'il (ou elle) a tenté d'exprimer, il faut attirer l'attention du lecteur vers ce que l'ouvrage nous semble comporter de plus stimulant, de plus provocant, de plus enrichissant. Lorsque j'ai recommandé ce travail à JeanMarie Tremblay pour qu'il le rendre accessible au public et qu'après avoir accepté, il m'a demandé d'en rédiger la préface, je me suis trouvé confronté à un dilemme assez particulier. Comme le titre de l'ouvrage l'indique bien, l'auteure, Brigitte Fleury, y rend compte de ses recherches, réflexions et lectures sur le déroulement du processus de conversion religieuse; processus qui pourrait d'ailleurs tout aussi bien être celui d'une « révolution paradigmatique» (pour employer l'expression de Thomas Kuhn) propre aux démarches scientifiques créatrices. Ce qu'il y a de particulier ou même de «métacommunicationnel » ici est que l'auteure peut donner l'impression d'y effectuer, elle-même, une conversion conceptuelle en s'inscrivant dans une façon de 
concevoir la communication qui est assez originale. Comme cette approche est aussi celle que je m'efforce de promouvoir dans mes écrits et enseignements, pendant quelques secondes, on pourrait croire que je l'aie « convertie »!

Heureusement, tout chercheur universitaire se doit d'être très modeste. Avec l'âge, je crois y être parvenu. Je ne suis ni un gourou et quête d'adeptes et, surtout, Brigitte Fleury n'a rien d'une fanatique recrue d'une secte occulte ! Néanmoins, cette métaphore (qui se veut très éphémère), inspirée par le sujet de l'ouvrage qui suit, m'est venue à l'esprit en constatant l'ampleur des similitudes qu'il me semble y avoir entre nos façons d'exprimer ce que nous comprenons de la communication humaine ainsi que des différentes façons de l'appréhender.

Brigitte Fleury est certainement l'une des étudiantes sinon l'étudiante qui a le plus pris le temps et a consacré le plus d'énergie à explorer en profondeur les pistes de réflexions et de recherches que je propose dans mes écrits et enseignements qui, dans une large mesure s'inspirent des Écoles pragmatiques américaines. Non seulement, par ses lectures, elle couvre les principales références bibliographiques que j'utilise mais elle les a complétées et amplifiées tout au long de sa scolarité de maîtrise ainsi qu'au cours de la préparation du mémoire qui constitue cet ouvrage. En plus d'être entrée à fond dans le cadre théorique que certains qualifient d' «interactionisme symbolique » ou encore d' « approche systémique symbolique » tout en l'enrichissant d'apports anthropo-historiques humanistes contemporains et européens empruntés à Laplantine, Todorov et Zeldin, Brigitte Fleury a suivi la démarche méthodologique qui découle logiquement de ces courants ainsi que des principales réflexions sur les bouleversements paradigmatiques : le récit de vie et plus particulièrement le récit de vie de la chercheure.

C'est d'ailleurs en partant des réflexions que lui ont inspiré l'auto-analyse (« selfreflexivity » d'Anthony Giddens ou le « télésitisme » de Lee Thayer ou, tout simplement, l'introspection des Montaigne, Proust et Edgar Morin) de son itinéraire avant de se pencher sur celui de Garaudy que l'on peut constater que, dans son cas, il n'y a pas eu de véritable conversion à un nouveau paradigme mais plutôt formulation aboutie du fruit de son expérience et de ses réflexions par le biais du cadre théorique qu'elle a rencontré dans l'enseignement que je lui ai prodigué ainsi que dans ses propres lectures complémentaires.

Élevée dans le Catholicisme, elle a suivie la Révolution Tranquille du Québec en en adoptant les nouveaux idéaux et, surtout, par ses voyages et insertions réussies dans différentes cultures très étrangères à sa communauté d'origine, d'appartenance et d'interprétation, elle a été amenée à prendre les distances nécessaires à la pratique du télésitisme, de l'auto-réflexion ou de l'introspection qui lui ont permis de comprendre l'Autre, l'Étrange tant dans sa continuité que dans ses éventuels rebondissements, ruptures, rédemptions ou «résiliences » (pour reprendre cette expression Judéo-américaine que Boris Cyrulnik a récemment réintroduit dans le vocabulaire français !). Elle s'était donc bien équipée pour appréhender la complexité et les diffi- 
cultés de la communication humaine avant d'entreprendre sa maîtrise dans ce domaine. Brigitte Fleury, comme j'ai eu le plaisir de le constater, est l'une de ces rares étudiantes (ou chercheur) qui s'est donnée cette "variété requise » si chère au cybernéticien Ashby (auteur de « la loi de la variété requise » selon laquelle aucun système ne peut appréhender une complexité qui soit supérieure ou différente de la sienne) à partir de laquelle des phénomènes de communication aussi complexes que la conversion religieuse ou le changement de paradigmes scientifiques peuvent être compris.

Fidèle à son propre itinéraire et au cadre théorique qu'elle a logiquement adopté, elle a étudié le cas de la conversion d'un personnage qui a, lui-même, fait rendu compte de la façon dont il a vécu sa conversion. Au moins par deux de ses principaux ouvrages, Roger Garaudy s'est efforcé de rendre compte de sa conversion à l'Islam.

Le lecteur constatera peut-être la quasi absence d'éloges ou même de descriptions de l'Islam dans ce que Brigitte Fleury a retenu des travaux de Garaudy. Il semble que l'attrait de cette religion, généralement peu prisée des Occidentaux, provient chez Garaudy de la valorisation de ses propres origines biologiques, sa grand-mère qu'il estimait beaucoup, était «Mauresque ». Mais, c'est surtout le comportement héroïque et juste de soldats musulmans de l'armée française d'Algérie qui, durant la seconde guerre mondiale, avaient refusé d'ouvrir le feu sur lui et certains de ses camarades alors que leurs officiers, collaborant avec la métropole occupée par les Nazis, leur en avaient donné l'ordre qui semble l'avoir profondément influencé.

L'amour de sa grand-mère arabe et la reconnaissance qu'il éprouve envers ces Musulmans qui ont refusé de lui enlever la vie sont deux sentiments qui vont finir par triompher de ses très longues et infructueuses tentatives de conciliation des Catholiques et des Communistes incarnant, à ses yeux, l'institutionnalisation de deux systèmes de valeurs qui lui semblaient pourtant parfaitement compatibles. Les brutalités et la lâcheté des uns et des autres ont fini par avoir raison de ses tentatives de conciliation dignes du mythe de Sisyphe. Ses idéaux humanistes ont finalement trouvé refuge en l'Islam ou les principaux officiants qu'il y rencontre l'adoptent en tant que penseur et écrivain converti.

Évidemment, dans ce travail, reste occultée toute la question de l'antisémitisme dont Garaudy a été accusé bien après sa conversion à l'Islam. Compte tenu de l'aspect très délicat et dangereux de ce sujet, Brigitte Fleury a justement décidé de ne pas aborder cet aspect de l'œuvre de l'auteur puisque ce «dérapage » s'est produit bien après la conversion de Garaudy à l'Islam et que, seul, ce processus faisait l'objet de son mémoire.

Aussi plutôt que de s'empêtrer dans les aspects les plus contestés et hors sujet des derniers travaux de Garaudy, Brigitte Fleury a méticuleusement expliqué les éléments et les rouages conceptuels des mécanismes de la communication qui sont à l'œuvre 
dans l'ouverture de l'esprit d'une personne à d'autres cultures, à d'autres visions du monde et de l'au-delà !

On peut donc dire que Brigitte Fleury, par son excellent travail de réflexion et de documentation pluridisciplinaire, nous offre plus qu'un outil, plus qu'une approche, plus qu'une théorie, en fait: un paradigme pour l'étude des phénomènes les plus énigmatiques du champ d'études de la communication humaine. Comprendre l'Autre - lorsqu'il est confronté à la société et aux communautés qui sont sensées le façonner et surtout lorsqu'il réussit à résister en s'ouvrant à l'étranger - procure une satisfaction intellectuelle qui ne peut que générer joie et espoir. Incontestablement, par ce mémoire, Brigitte Fleury ouvre une brèche dans le mur de l'enfermement identitaire et conceptuel!

René Jean Ravault, Ph.D.

Professeur associé

à l'École de Communication

de l'UQÀM. 


\section{Remerciements}

J'ai bénéficié de la contribution de plusieurs personnes pour réaliser le présent travail. Je tiens, tout d'abord, à remercier mon directeur de mémoire et professeur, René-Jean Ravault, dont l'originalité des idées m'a profondément inspirée et qui a su me guider dans l'apprentissage d'une nouvelle vision du monde; également les professeurs(es) Luce Desaulniers, Claude-Yves Charron, Jacques Rhéaume et Philippe Sohet qui, chacun à sa manière, a su stimuler une réflexion créatrice chez moi ; Monic Paysan, Denise Chartrand et Benoît Chartrand, pour l'aide familiale qu'ils m'ont apportée ; enfin, mes enfants Émile et François-Oliver, qui ont été une source constante de motivation. L'appui de toutes ces personnes a été très précieux et je leur en sais gré. 


\section{Résumé}

Les recherches portant sur l'étude des conversions religieuses ont tenté, dans bien des cas, d'identifier les causes objectives du phénomène. Cette façon de procéder occulte, toutefois, le point de vue du converti, plus précisément comment il en vient à concevoir sa conversion. Aussi, dans ce mémoire, nous nous interrogeons sur la construction des significations par les personnes. Nous explorons comment les enfants se socialisent et finissent par adopter une vision du monde en conformité avec les attentes de leurs parents. Cette opération contribue à définir l'identité de la personne, c'est-à-dire ce qui est significatif pour elle. Les études de «l'École de Columbia » ont démontré qu'il en va sensiblement de même à l'âge adulte. Les personnes s'identifient aux propos de ceux en qui elles ont confiance. Mais alors comment est-il possible pour un individu de renoncer aux significations religieuses qu'il a acquises au sein de sa communauté d'appartenance pour en venir à en adopter d'autres auprès de membres d'un autre réseau de relations ? Nous pensons qu'il s'agit d'un processus communicationnel complexe élaboré au fil des expériences d'une vie. Tout d'abord, l'humain peut réfléchir sur sa condition, communiquer avec lui-même afin de revisiter la façon dont il appréhende ses relations avec l'extériorité, mais aussi avec les autres. De plus, il s'unit à des réseaux de connaissances avec lesquels il partage des valeurs et des projets communs. Or, des désaccords peuvent survenir entre les membres d'un groupe à propos de l'interprétation des situations qui se présentent. Cette situation peut inciter à la conversion en autant que la personne soit ouverte à 
l'alternative. Dans bien des cas, l'adhésion à un nouveau groupe procurera des privilèges au nouveau converti. C'est ce que nous avons pu constater dans notre étude de cas portant sur la conversion de Roger Garaudy. Les personnes ne se convertissent pas uniquement au contact des autres ou à la suite de la lecture d'un Livre saint. Pour effectuer un tel changement, il faut que la personne puisse percevoir, dans ses relations avec ses proches, des difficultés à s'accorder sur la signification des termes échangés. Cette situation peut stimuler la volonté de s'entendre avec d'autres sur l'assignation d'un rapport au monde différent.

Mots clé : conversion, religions du Livre, construction de la « réalité », identité, communautés d'interprétations, communisme, réception active, réflexivité, télésitisme, communication intrapersonnelle, communication interpersonnelle. 


\section{Introduction}

«We are what we think.

All that we are arises with our thoughts.

With our thoughts we make the world. » ${ }^{1}$

$\underline{\text { Retour à la table des matières }}$

Une des explications courantes évoquées à propos des cas de conversion religieuse consiste à attribuer la cause d'un tel phénomène au fait d'être exposé à un discours, écrit ou oral. On dit de l'adepte religieux qu'il s'est converti, par exemple, à la suite d'une lecture du Coran. Ou encore, on le perçoit comme une victime qui subit l'ascendant d'une personnalité charismatique. Cette dernière, tel un aimant, semble exercer un pouvoir d'attraction qui lui permet d'endoctriner des personnes influençables. Faisant usage de ses dons oratoires, mettant à profit des techniques de persuasion, elle exhibe une force de leadership qui incite les autres à l'imiter en adoptant les mêmes pratiques en matière de religion.

Ces interprétations du phénomène de conversion, parce qu'elles impliquent la capacité de voir et de concevoir les choses autrement, relèvent de la notion de persuasion qui est au cœur de la réflexion en communication. De fait, les objectifs de la recherche traditionnelle dans ce domaine d'études consistent à expliquer « l'impact » $\mathrm{du}$ «message » d'un agent-émetteur sur un agent récepteur.

Lee Thayer, Pieces: Toward a Revisioning of Life Communication/Life, Greenwich (Con.) et London (Eng.), Ablex, 1997, p. 9. 
La description de ce phénomène a été inspirée par la célèbre formule proposée par Lasswell, pour synthétiser le processus étudié en son temps, aux États-Unis. Répondant aux questions «qui dit quoi, par quel canal, à qui, avec quels résultats », les chercheurs ont voulu déterminer les facteurs contribuant à la persuasion de l'auditoire. Ils se sont ainsi attardés, à étudier les caractéristiques sociales des agentsémetteurs ainsi que les contenus médiatiques. ${ }^{2}$ Mais si, dans certains cas, comme celui de l'étude portant sur la diffusion de l'œuvre d'Orson Wells, War of the Worlds, ils ont pu observer des effets spectaculaires appuyant leurs hypothèses, dans d'autres, le résultat escompté ne s'est pas produit; parfois même, il est allé à l'encontre de ce qu'ils avaient supposé. On parle alors d'effet boomerang. ${ }^{3}$

De notre point de vue, et en regard du phénomène de conversion religieuse, la possibilité de l'effet mitigé ou opposé est tout à fait concevable, car notre expérience personnelle en témoigne. Ayant vécu avec une musulmane pendant près de huit mois lors d'un échange culturel et ayant séjourné dans une famille de même confession au sein d'une petite communauté fervente de l'islam, nous n'avons jamais senti leur emprise sur nous. ${ }^{4}$

Nous ne nous sommes pas convertie à la suite de ces rencontres privilégiées avec des musulmans, pas plus que nous les avons jugés négativement. Dans l'ensemble, nous pouvons affirmer avoir effectué, à leur contact, une prise de conscience notable qui a contribué à nous révéler la spécificité du schème d'appréhension avec lequel nous interprétons notre « réalité » propre. Cela nous a permis également de réaliser la relativité des visions du monde. De plus, au cours de cette expérience, nous avons découvert que les modes d'interprétation des réalités sont aussi justifiés par des présupposés éthiques qui peuvent s'avérer similaires chez des personnes d'origines très différentes. Ainsi, la rencontre de l'autre et la prise en considération de son discours ne signifient pas nécessairement que l'on adopte en tout point sa vision du monde. On peut toutefois saisir, au-delà des différences, l'universalité de certains de ses propos.

Nous supposons donc que la conversion religieuse n'est pas subie par le sujet, mais qu'elle constitue plutôt un processus par lequel il s'investit volontairement. C'est pourquoi nous estimons qu'il s'agit beaucoup plus d'une démarche motivée par

2 John W. Riley jr. et Matilda W. Riley, «La communication de masse et le système social», In Sociologie de l'information, sous la dir. de F. Balle et J. G. Padioleau, Paris, Larousse, 1973, pp. 70-71.

Ibid., p. 73.

L'échange dont il est question se nomme « Jeunesse Canada Monde » et nous y avons participé durant l'année 1985-1986. Notre groupe était constitué de 7 participants indonésiens jumelés à 7 participants canadiens et supervisé par un agent de groupe canadien et un agent de groupe indonésien. Nous avons séjourné chez une famille établie à Victoria, en Colombie-Britannique, pendant quatre mois, puis, pendant quatre autres mois nous avons été hébergés dans une famille, mais cette fois dans la petite communauté de Lubuk Mumpo, sur l'île de Sumatra, en Indonésie. Nous avions pour tâche d'effectuer autant au Canada qu'en Indonésie des travaux communautaires. 
des difficultés à assigner des significations habituelles, à des expériences désormais perçues sous un jour nouveau.

Envisagé sous cet angle d'analyse, l'acte de conversion devient une recherche destinée à remédier à cette situation. Nos propos ne se réfèreront donc pas à ces conversions massives qui s'effectuent sous la contrainte dans des contextes particuliers de guerre ou de dissolution de l'environnement social.

L'image du converti que nous gardons tout au long de l'écriture de ce mémoire est celle d'un être qui ne se sent pas victime des autres ou des circonstances, mais agit et réagit, selon sa conscience, en fonction de la mouvance des êtres et des choses qui l'entourent. En termes communicationnels, nous parlerons donc d'un récepteur actif ; c'est-à-dire d'une personne qui, selon son bagage socio-culturel et les variations de son interprétation du monde dans le temps et l'espace, pourra interpréter les stimuli extérieurs de façons multiples. C'est cet ensemble de variables à décoder qui a des conséquences très complexes sur le plan de la perception, de la cognition, de la communication intrapersonelle et interpersonnelle ainsi que sur les comportements, que nous voulons examiner de plus près.

Si l'approche utilisée ici pour comprendre le phénomène de la conversion religieuse cherche à mettre de l'ordre dans un enchevêtrement de facteurs multiples, nous ne souhaitons pas endosser une démarche néo positiviste. D'une part, nous ne prétendons pas que l'on puisse isoler l'objet de recherche de toutes contingences, qu'elles soient d'ordre contextuel ou associées à l'observation du phénomène. ${ }^{5}$ C'est pourquoi, nous ne cherchons pas, conformément à la conception de la science « classique », à repérer, définir, puis mesurer par l'intermédiaire d'entrevues répétées, les facteurs qui interviennent dans l'explication du phénomène. De plus, nous ne souscrivons pas à la pensée aristotélicienne selon laquelle l'apparition d'une contradiction constitue une erreur logique. Selon nous, une contradiction ne constitue pas nécessairement une réfutation. Elle démontre plutôt la nature complexe de notre humanité. D'autre part, il ne nous semble pas que l'expérience de conversion nous soit plus compréhensible en nous intégrant personnellement, mais sans y adhérer, à un groupe de personnes qui s'investit dans cette démarche. Selon nous, l'expérience de conversion ne se réduit pas à un moment ou à une expérience, mais consiste plutôt en une interprétation qui est revue au fil de l'expérience communicationnelle des personnes. Chaque être humain se parle constamment et interagit avec les autres. Cette activité personnelle et collective facilite, chez l'individu, la mise à jour des prises de conscience sur son monde en perpétuel changement. Par conséquent, l'expérience de la conversion religieuse s'élabore en autant qu'il arrive à lui donner un sens pour lui, mais aussi avec ceux en qui il a suffisamment confiance pour étaler cette possibilité.

De plus, la construction de ce type d'expérience est exigeante parce qu'elle signifie aussi que les personnes en viennent non seulement à reformuler leurs croyances,

5 Edgar Morin, Science avec conscience, Paris, Fayard, 1982, p. 295. 
mais aussi leur adhésion à un groupe. Cette possibilité procède aussi de la faculté chez l'humain de pouvoir affronter ses contradictions. Les comportements humains ne peuvent être cernés et identifiés de façon invariable ; l'humain possède la faculté de réfléchir sur lui-même, de s'auto-évaluer et de changer. Aussi cherchons-nous à explorer les interactions communicationnelles dans lesquelles il s'investit afin de rendre compte de sa «réalité » conformément à son adhésion à une nouvelle vision du monde. Plus précisément, nous voulons nous concentrer sur l'explication du phénomène de la conversion religieuse en relation avec le processus de réception active.

Plusieurs études ont cherché à identifier les processus psychologiques des sujetsrécepteurs ainsi que leurs prédispositions à accepter un discours nouveau par exposition à des produits médiatiques. Soulignons l'étude d'Herzog où des chercheurs ont constaté que beaucoup de femmes écoutent des romans-feuilletons diffusés le jour parce qu'elles recherchent des modèles nouveaux de solutions à des problèmes quotidiens ${ }^{6}$ De plus, selon Berelson, Herzog, Lazarsfeld et Robinson, pour qu'un discours influence une personne, il doit évoquer un registre de questionnement qui suscite son intérêt. Dans le cas contraire, elle n'y prêtera pas attention. ${ }^{7} \mathrm{Et}$, dans le cas où un sujet-récepteur accepte de considérer ce qu'on lui propose, il peut sélectionner certains segments particuliers, ou encore s'investir dans des degrés variables de concentration dans cette activité. De plus, les personnes ont tendance à interpréter ce qui les intéresse en fonction de ce qu'elles veulent croire. L'humain prend connaissance de son monde non pas tel qu'il est, mais plutôt comme il veut le percevoir et le concevoir. L'acte perceptif fluctue en fonction des valeurs, des émotions et des expériences passées des personnes. L'être humain a tendance à conformer les stimuli qu'il note à sa vision des choses. Des stimuli identiques peuvent être interprétés différemment dépendamment de la classe sociale, de la culture, de la religion, etc., auxquelles une personne adhère. Il en résulte des explications variées. Des personnes vont ainsi ignorer certaines informations, d'autres vont les réorganiser de façon à ce qu'elles correspondent à leurs intérêts, à leur goût et à leurs croyances. On parle alors de dissonance cognitive. Cette situation est attribuable au fait qu'il existe un rapport entre l'orientation de la perception des individus et la structure sociale à laquelle ils participent. En effet, les sujets-récepteurs ont tendance, peu importe l'idéologie prédominante à un moment dans les débats publics, à adopter les mêmes réponses que leurs semblables, c'est-à-dire leur famille, leurs amis ainsi que toutes les personnes qu'ils jugent importantes pour elle.

Pour Peter Berger et Thomas Luckmann, un acte d'infirmation de la « réalité » exécuté par un proche que l'on respecte et en qui on a confiance, plutôt que par une connaissance fugitive, risque d'être plus persuasif. ${ }^{8}$ Katz et Lazarsfeld en viennent

6 John W. Riley jr. et Matilda W. Riley, «La communication de masse et le système social», In Sociologie de l'information, sous la dir. de F. Balle et J. G. Padioleau, Paris, Larousse, 1973, pp. 74-75.

7 Ibid., pp. 74-75.

8 Peter Berger et Thomas Luckmann, La construction sociale de la réalité, Paris, Méridiens Klincksieck, 1986, p. 207. 
sensiblement à la même constatation. Malgré la présence de divers points de vue dans les sociétés démocratiques, l'humain ne se laisse pas envahir par tous les discours. Étant le plus souvent dans l'impossibilité de faire abstraction de ses attachements identitaires, c'est-à-dire du mode d'appréhension avec lequel il attribue des significations à son monde, il s'investit dans la sélection d'informations qui lui conviennent, en provenance des autres. Ces chercheurs ne parlent pas de sujets qui subissent l'influence des autres. Ils les conçoivent plutôt comme des acteurs sociaux qui participent activement selon leurs identifications multiples à l'élaboration de discours communicationnels. Cette activité témoigne de leur adhésion à leur communauté d'interprétation. ${ }^{9}$

En d'autres termes, la communauté des valeurs vécues et véhiculées à l'intérieur des groupes primaires comme la famille et les amis ou encore celle qui oriente le discours des divers groupes d'affiliation secondaires qu'il soit d'ordre professionnel, syndical, politique ou religieux, serait loin d'être battue en brèche dans la société moderne [...] ${ }^{10}$

Les comportements des individus correspondraient plutôt aux systèmes de valeurs qu'ils auraient assimilées au contact des membres de ces groupes. Ainsi, selon l'intériorisation de la conscience collective, les actions conformes à la norme inculquée seraient soutenues, tandis que celles qui y dérogent risqueraient la désapprobation. Cette interprétation s'est trouvée corroborée par une étude menée à l'université Rutgers et qui confirme que, plus un enfant communique avec ses parents, plus il adhère à leur système de valeurs et plus son interprétation ainsi que son choix des médias vont se conformer aux attentes parentales. ${ }^{11}$

Par conséquent, la prise en compte d'une nouvelle vision du monde qui met en doute les habitudes acquises et partagées au sein des groupes de références primaires, constitue une démarche exigeante et plutôt inhabituelle. De façon générale, très peu de personnes migrent d'une communauté de croyances à une autre parce que cette démarche nécessite une remise en question des présupposés qui conditionnent l'interprétation de chacun, d'où beaucoup d'incertitude et de questionnement. Des recherches conduites par Newcomb et Festinger ont d'ailleurs démontré qu'un membre ré-

9 Lee Thayer, On Communication : Essays in Understanding, Norwood, (New Jersey), Ablex, 1987, p. 49.

10 Dans ce travail, nous allons utiliser les termes communautés d'appartenance pour faire référence aux groupes primaires. De plus, pour faciliter la compréhension du texte, nous désignerons les groupes d'affiliation secondaires par les termes, communautés épistémiques. Ces deux types de communautés seront également considérés, tout au long du texte, comme des communautés d'interprétation. La citation est de Elihu Katz, «Les deux étages de la communication (The twostep flow of communication)», In Sociologie de l'information, sous la dir. de F. Balle et J. G. Padioleau, Paris, Larousse, 1973, p. 285.

11 Pour en savoir plus lire Matilda White Riley et John Riley, jr, «A Sociological Approach to Com-munication Research», Pub. Opin. Quart., 72, 13, 1951, pp. 445-60, ainsi que Matilda White Riley et Samuel H. Flowerman, «Group Relations as a variable in Communications Research», Amer. Sociol. Rev., 16, 1951, pp. 174-80. Ces informations ont été prises dans John W. Riley jr. et Matilda W. Riley, «La communication de masse et le système social», In Sociologie de l'information, sous la dir. de F. Balle et J. G. Padioleau, Paris, Larousse, 1973, p. 79. 
fractaire à une communauté d'appartenance ou de pensée ne se convertirait pas, tant que les raisons qui le poussent à rester au sein du groupe revêtent de l'importance pour lui. ${ }^{12}$ Par contre, le poids de ces opinions diminue si ce membre perçoit d'autres groupes qui répondent à ses attentes tout en endossant sensiblement les mêmes valeurs et attitudes. En outre, des données recueillies par des chercheurs de « l'École de Columbia » sur les décisions de vote de l'élection présidentielle de 1940, ont démontré que les leaders d'opinion sont choisis, non seulement en fonction de leurs caractéristiques socio-démographiques (statut social, sexe, âge, etc.), mais aussi pour la structure et les valeurs du groupe auquel ils appartiennent. ${ }^{13}$ Bien que ces personnes orientent l'opinion dans certains domaines, elles peuvent elle-même subir l'influence des autres dans des champs différents. Elles semblent d'autant plus convaincantes qu'elles disposent d'information supplémentaire, ce qui leur permet de mieux étoffer leur propos.

Nombre de recherches sur la conversion religieuse ont ignoré les constats que nous venons d'énoncer. Et c'est sans doute cela qui explique la perception répandue que les convertis sont «victimes » des influences des autres. Pour notre part, la prise en compte de ces informations a changé notre façon d'appréhender ce phénomène. Aussi voulons-nous présenter, dans ce mémoire, la construction communicationnelle et volontaire d'une telle expérience. Bien que nous reconnaissions que l'expérience et l'interprétation de chaque personne soient en partie déterminées par leurs références culturelles et éducationnelles, nous estimons que la conversion exprime la possibilité chez l'humain, contrairement à l'animal, de se détacher de ses conditionnements et d'explorer sa volonté de liberté en cherchant à reconnaître une vision nouvelle du monde qui fasse sens et qui lui permette de s'associer aux autres dans l'élaboration de nouveaux projets. Nous espérons qu'en démystifiant la façon dont les gens en viennent à remettre en question leur appartenance religieuse pour en adopter d'autres, ils ne seront plus perçus comme des «transfuges», mais plutôt comme des « émissaires » qui possèdent l'avantage de circuler dans deux univers de signification,

12 Pour en savoir plus, lire les articles de Theodore M. Newcomb, «An Approach to the Study of Communicative Acts», Psyco. Rev., 60, 1953, p. 393-404, et de Leon Festinger, «Informal Social Communication», Psycho. Rev., 1950, pp. 271-282. Ces informations ont été prises dans John W. Riley jr. et Matilda W. Riley, «La communication de masse et le système social», In Sociologie de l'information, sous la dir. de F. Balle et J. G. Padioleau, Paris, Larousse, 1973, pp. 80-81.

13 Nous nous référons ici aux études de Merton portant sur l'influence interpersonnelle à Rovere ; celle de Decatur, menée par Katz et Lazarsfeld et traitant de la formation des décisions dans différents secteurs d'activité ; celle d'Elmira, élaborée par Berelson, Lazarsfeld et MacPhee analysant l'influence sur le vote lors de la campagne électorale de 1958 ainsi que celle de Coleman, Katz et Menzel sur la diffusion d'un nouveau remède par les médecins. Nous voulons également préciser que les groupes primaires correspondent aux organisations sociales qui s'imposent à l'individu pendant son enfance, par exemple la famille, tandis que les groupes secondaires s'apparentent aux communautés épistémiques auxquelles il peut adhérer éventuellement par choix dans le cours de sa vie adulte. Elihu Katz, «Les deux étages de la communication (The two-step flow of communication)», In Sociologie de l'information, sous la dir. de F. Balle et J. G. Padioleau, Paris, Larousse, 1973, p. 287. 
et qui, partant, peuvent faciliter la communication entre les tenants de visions du monde incommensurables.

Comme nous n'avons pas trouvé d'auteurs qui abordent l'étude des conversions religieuses sous cet angle d'analyse, notre exposé s'apparente beaucoup plus à une démarche exploratoire qui cherche à susciter une perception renouvelée, voire humaniste, de la façon dont cet objet d'analyse peut être appréhendé. Aussi, pour atteindre notre objectif, allons-nous mettre en relation des concepts variés et les présenter de façon parfois inusitée.

Cette démarche n'est pas sans lien avec qui nous sommes en tant que chercheur, ainsi que notre parcours de vie. C'est pourquoi nous exposerons un peu plus loin dans ce mémoire, la nature des références qui sont, pour nous, importantes dans l'appréhension du monde. Ceci expliquera, en partie, ce qui nous à motivé à entreprendre l'écriture de ce mémoire. Par ces explications, nous souhaitons également démontrer comment nos projets sont élaborés en relation avec la communication intra-personnelle que nous entretenons avec nous-mêmes.

Mais avant d'aborder ce sujet, et pour comprendre son importance, nous devrons expliquer comment l'appréhension de la «réalité » se fait selon des modes de représentations propres à l'identité de chacun. Nous verrons ensuite que cette identité tend à se préserver auprès de ceux et celles qui partagent des représentations similaires, c'est-à-dire au sein des groupes d'appartenance puis auprès des groupes d'affiliation. De plus, si la conversion constitue un phénomène inusité - puisque la personne consent à remettre en question, en partie ou de manière plus extensive, son attachement identitaire antérieur - nous voulons démontrer comment ce compromis est négocié au sein des échanges intra-personnels et interpersonnels. Enfin, considérer la conversion comme une initiative qui en vaut la peine ne survient pas instantanément, peu importent les apparences. L'apprentissage d'une nouvelle vision du monde, qui peut conduire à l'intégration d'une nouvelle communauté se construit, dans le temps ou l'espace, au contact d'un réseau de relations de plus en plus significatif pour la personne qui se convertit.

Nous tenons à souligner que le point de vue développé dans ce travail, intègre bon nombre de considérations théoriques, et accorde une attention particulière aux cas de conversion dont nous avons pris connaissance, notamment des conversions à l'islam. Cela est sûrement attribuable au fait que nous comptions procéder à une étude de cas, en dernière partie de ce travail, qui témoigne de ce type de conversion ; il s'agit de l'expérience de Roger Garaudy qui a adhéré à l'islam après avoir longtemps milité comme Marxiste et Catholique au sein du Parti communiste français. Nous souhaitons, par cet exercice, rendre plus accessible l'ensemble des notions théoriques qui auront été préalablement développées.

De plus, nous avons tout au long de ce travail considéré le converti comme l'acteur de sa propre conversion. Nous voulions ainsi démontrer comment il contri- 
bue, en tant que récepteur-actif, à l'élaboration de son expérience de conversion. Pour arriver à développer cette perspective d'analyse, nous avons pensé qu'une étude de cas en profondeur nous permettrait de relever au travers du temps cette construction. Il nous est apparu également, qu'en limitant notre étude des œuvres de Garaudy nous pourrions saisir avec plus de perspicacité les facteurs qui ont contribué à la fondation de ses représentations et noter les changements qui ont contribué à modifier leur interprétation. Pour ce faire, nous avons tenu compte de deux monographies de son œuvre qui nous semblent les plus significatives en ce qui a trait à l'objet de notre recherche. Il s'agit de son Testament philosophique ainsi que de ses Mémoires autobiographiques. ${ }^{14}$ Nous avons estimé qu'en procédant ainsi nous pourrions parvenir à développer la construction communicationnelle de l'expérience de conversion. D'abord parce que dans les œuvres choisies, Garaudy fait le point sur sa vie personnelle ainsi que sur son discours philosophique. Il prend une distance par l'écriture (plus grande au début et plus petite à la fin), sur les préoccupations qui l'ont animées pendant le cours de sa vie ainsi que les relations et organisations qui ont été importantes pour lui dans son cheminement. Ces œuvres nous permettent de saisir la conversion religieuse non pas comme un événement particulier, mais comme une longue séquence d'événements, de réflexions et de rencontres qui comportent aussi des moments charnières. Mais en même temps, elles livrent tout un bagage d'information qui n'est pas si facilement accessible à première lecture, ne serait-ce que parce nous n'avons pas vécu au même moment et endroit que lui. Se rapprocher des motivations qui dirigent l'interprétation n'est pas une tâche facile. Essayer de comprendre comment une personne en vient à concevoir son univers implique de s'attarder sur les événements historiques marquants pour elle, les personnes qui l'ont influencées, les débats d'idées qui l'ont occupés pendant de longues périodes de temps, ainsi que les groupes dans lesquels elle s'est investie et où elle y a adapté son discours aux changements. La recherche et la compréhension des éléments qui semblent importants pour une personne dans son appréciation du monde peuvent expliquer les variations d'interprétation relatives à son univers communicationnel particulier.

En regard de l'analyse que nous avons développée, nous émettons toutefois certaines réserves. Afin de démontrer la validité de notre propos, nous avons peut-être eu tendance, parfois, à trop vouloir faire correspondre certains extraits aux hypothèses et acquis conceptuels présentés. De toute évidence la grille d'interprétation que nous avons utilisée dans ce travail est sommaire. L'emphase a été mise sur l'articulation sociologique et communicationnelle de certains processus, plus précisément la construction sociale des identités personnelles et sociales et des systèmes de représentations et de valeurs. Ce cadre conceptuel nous a permis de développer les fondements communicationnels et interactifs à l'œuvre dans le processus de socialisation. D'autres cadres d'analyse, telle la psychanalyse, offrant des outils conceptuels pour comprendre sur une longue période de temps l'expérience du sujet auraient pu offrir

14 Les références complètes de ces livres sont : Roger Garaudy, Biographie du XXe siècle : Le testa-ment philosophique de Roger Garaudy, Paris, Tougui, 1985, et Mon tour du siècle en solitaire : Mémoires, Paris, Robert Laffont, 1989. 
des résultats différents et tout aussi pertinents. Nous n'ignorons pas que notre recherche découle de notre interprétation des choses et que notre compréhension de la communication est influencée par les échanges que nous concevons dans nos relations, par le biais de nos lectures, somme toute qui nous sommes. Nous sommes tout à fait consciente que notre réflexion est partielle et singulière. C'est le premier pas d'une démarche qui mérite d'être enrichie et complétée par d'autres points de vue.

Tout comme nous concevons les limites la présente analyse, nous tenons également à émettre certaines réserves à propos de la personne dont il est question dans notre étude de cas. Si les propos de Roger Garaudy ont été sujets à controverse parce que certaines de ses positions ont mis en doute des faits relatifs à l'holocauste juif durant la Seconde Guerre mondiale, nous tenons à souligner que nous ne cautionnons pas l'idéologie révisionniste. Nous nous référons à Roger Garaudy simplement parce qu'il est possible, par l'intermédiaire de ses œuvres, de suivre le cheminement qui l'a conduit à se convertir.

De plus, la pertinence de notre exposé ne repose pas sur le nombre de cas étudiés, mais sur la qualité de l'analyse à rendre compte du processus de façon nuancée et selon les présupposés des recherches sur la réception active. La façon dont nous abordons cet objet de recherche nous apparaît justifiée parce que les informations disponibles sur le phénomène des conversions religieuses ne consistent actuellement qu'en une collection de points de vue spécifiques. Aucun consensus ne semble établi quant à la nature même du processus et du fil conducteur de ce type d'expérience.

C'est pourquoi nous expliquons, dans le chapitre 1, les lacunes des diverses analyses de la conversion religieuse traitées sous l'angle de la sociologie, de la psychologie, de l'histoire, et de l'anthropologie. Bon nombre de ces recherches tentent d'exposer les conditions objectives qui expliquent le phénomène de conversion. Dans le présent travail, nous développons l'idée que la conversion survient par ce qui est dit et construit dans le discours de la personne et découle, par conséquent, de son interprétation subjective. Toutefois, la conversion implique aussi pour la personne, de remettre en question sa vision du monde et ses attachements identitaires. Cette prise de conscience ne va pas de soi et nous nous demandons comment ce phénomène se produit.

Dans le chapitre 2, nous expliquons la distribution sociale de la connaissance et son appropriation par les individus. Nous examinons comment ce processus s'élabore sous l'angle de la réception communicationnelle.

Dans le chapitre 3, nous explorons l'univers communicationnel de Roger Garaudy afin d'établir comment il a construit le sens de sa conversion religieuse à l'islam.

Enfin, dans la conclusion, nous dégageons les éléments qui nous ont paru significatifs dans notre recherche. 


\section{Chapitre I \\ Développement de la problématique}

« Il y a mille possibles en moi, mais je ne peux me résigner à n'en n'être qu'un seul. » 15

\section{1-1. Lacunes des analyses du phénomène de conversion religieuse}

Retour à la table des matières

Le phénomène de conversion a beaucoup été étudié en sociologie des religions. Bien que nous reconnaissions cet apport dans le traitement de la problématique relative aux conversions religieuses et notamment l'analyse des formes élémentaires de la vie religieuse élaborée par Durkheim, nous constatons, comme nombre d'auteurs, les limites de ce cadre d'analyse. Ces études, ont pris pour postulat que l'individu isolé ne réagit pas de la même façon que l'individu membre d'une organisation. Elles ont cherché à identifier les facteurs qui unissent ce dernier au groupe, qu'ils soient religieux ou autres. Mais ces interprétations de la conversion religieuse en tant que phé-

15 Cette citation d'André Gide est reprise par François Laplantine dans son livre Je, nous et les autres : Être humain au-delà des appartenances, Paris, le Pommier, 1999, p. 40. 
nomène sociologique rationnel produit par des causes impersonnelles et externes au sujet, ont démontré leurs limites. Elles ont négligé de considérer la force interne de mobilisation que représentent les croyances des individus investis dans ce type d'expérience.

Même les tentatives visant à élargir ce domaine d'études en empruntant des perspectives d'analyse à la psychologie, à l'anthropologie ou à l'histoire, n'ont guère été plus concluantes. Parce qu'il n'y a aucune vision de la conversion qui fasse consensus, on se retrouve avec des perspectives d'analyse propres à chacune des disciplines, avec des orientations particulières et des méthodologies sélectives. Cette situation a pour conséquence de produire des analyses trop spécifiques à la compréhension globale du phénomène. L'approche psychologique a conduit à la production d'analyses centrées sur les dynamiques émotionnelles en relation avec l'environnement familial ou social. ${ }^{16}$ L'approche anthropologique a produit maintes études sur l'impact culturel du prosélytisme missionnaire ainsi que les effets d'un tel processus sur le développement des communautés. Enfin, l'approche historique a souvent servi à relever les changements de motivation à se convertir selon les variations du contexte social dans le temps.

À ces études, s'ajoutent d'autres études portant sur :

1) l'effet d'attraction que suscitent des groupes religieux auprès de certains individus sensibles à la dimension spirituelle,

2) les facteurs socio-culturels prédisposant les individus à la conversion,

3) la compatibilité des croyances religieuses des grandes religions avec celles de systèmes préétablis de croyances. ${ }^{17}$

Dans l'ensemble, elles oublient de prendre en considération le point de vue subjectif de la personne qui s'investit dans une telle expérience. Ces études visent à élucider les conditions objectives qui président à la conversion des individus. Cette façon de concevoir le phénomène est en partie attribuable à l'infiltration de la pensée scientifique dans la culture moderne. Elle contribue à discréditer les explications des activités humaines non-conformes au paradigme cartésien de l'objectivité. ${ }^{18}$

16 Lewis R. Rambo, Understanding Religious Conversion, New Haven and London, Yale University Press, 1993, pp. 8-12.

17 Brian Taylor, «Conversion and Cognition, An Area for Empirical Study in the Microsociology of Religious Knowledge», Social Compass, XXIII, 1976/1, pp. 7-11.

18 James W. Carey, «Reconceiving "Mass" and "Media” », In Communication As Culture, Essays on Media and Society, New York, London, (Great Britain), Routledge, 1988, p. 72. 


\section{1-2 Le choix de l'approche constructiviste}

$\underline{\text { Retour à la table des matières }}$

Aussi, selon nous, l'explication d'un phénomène comme celui de la conversion ne réside pas uniquement dans l'expression objective d'une telle "réalité », mais plutôt dans l'interprétation subjective de la personne qui participe au développement d'une telle expérience. Nous acceptons, tout comme les structuralistes, que l'humain s'approprie des facettes de la culture ambiante et inversement que cette culture contribue à modifier ce qui est à l'extérieur de lui. Mais en plus, nous pensons qu'il contribue à façonner sa « réalité » et que cette activité le transforme à son tour. Ce mouvement perpétuel d'influences explique en partie pourquoi la «réalité » ne peut être considérée objectivement, à moins d'en soustraire l'appréhension humaine.

En d'autres termes, la façon dont l'esprit saisit la « réalité » et les répercussions qui s'ensuivent, tant au niveau de l'individu que du plan social, se constituent dans une relation dialectique. Ce mode d'appréhension contribue à la construction des significations individuelles et collectives. Ce processus révèle, des significations au fur et à mesure de son élaboration. On nomme l'approche qui considère ces présupposés : constructiviste. Elle présume que tous les individus, se définissent constamment les uns par rapport aux autres, parce qu'ils s'investissent à attribuer leurs significations au monde. Si objectivement il existe un monde dans lequel ils sont inclus, ils apprennent aussi à le nommer au contact des autres. Cette activité a pour conséquence d'engendrer des conceptions du monde qui façonne les individus sur le plan identitaire. On ne parle donc pas simplement de sujets qui subissent la « réalité », mais bien d'acteurs qui en fonction de leur identité interprètent et participent à la construction de réalités multiples.

Même si nous vivons avec l'illusion que la « réalité » semble procéder d'ellemême selon un ordre logique, la connaissance que nous en avons se construit socialement. Selon Peter Berger et Thomas Luckmann, la participation de chaque être humain à cet exercice s'effectue dans l'interrelation constante d'interactions se situant dans trois dimensions abstraites et conceptuelles désignées par les termes d'objectivation, d'intériorisation et de subjectivation.

L'objectivation prend en considération la notion de réalité sociale élaborée par Durkheim. L'intériorisation, fait référence à la dimension subjective travaillée par Weber tandis que la subjectivation, souligne l'apport de la perspective-dialectique développée par Marx. En d'autres termes, l'objectivation représente le rapport de soi 
avec les produits de l'extériorisation, c'est-à-dire la relation entre tout être humain et ce qui est produit dans le sillage de l'activité humaine, physique et mentale. L'intériorisation constitue le processus par lequel «[...] le monde social objectivé est projeté dans la conscience au cours de la socialisation [...] », ${ }^{19}$ alors que la subjectivation correspond à la relation entre l'intériorité de l'être et les objets de l'extériorisation, à ce va et vient incessant entre acteurs, en tant que producteurs de réalité sociale et produits de l'extériorisation. Selon cette perspective d'analyse, les individus dans leurs constantes interactions avec la nature, les êtres humains et leurs créations, participent à la construction de ce qu'ils sont et ce qu'ils font.

Par conséquent, la conversion, selon ce schème d'appréhension, ne se limite pas à un moment, celui du rituel d'intégration à une nouvelle communauté, et ne peut être considérée comme un processus historique unilinéaire. Elle se construit en un projet qui devient perceptible au fur et à mesure que les jeux et enjeux se précisent dans l'interaction avec les autres et le retour sur soi. D'où la difficulté pour bien des auteurs à définir l'objet d'étude dont il est question dans ce travail étant donné qu'il s'agit d'un processus en élaboration. ${ }^{20}$ C'est pourquoi la plupart des auteurs, confrontés à la relativité et à la complexité de l'objet de cette étude, vont s'entendre sommairement pour définir la conversion comme une transformation. Nous précisons pour l'instant qu'il s'agit beaucoup plus d'une altération identitaire qui survient à la suite des interactions communicationnelles auxquels nous participons. Nous allons brièvement expliquer cette conception de la conversion religieuse avant de démontrer en quoi elle est problématique. Mais pour commencer il faut expliquer le processus par lequel se construit l'identité afin d'expliquer par la suite sa transformation.

19 Peter Berger et Thomas Luckmann , La construction sociale de la réalité, Méridiens Klincksieck, Paris, 1996, p. 87.

20 Dans nos lectures, la plupart des auteurs qui abordent le phénomène de conversion reconnaissent cette difficulté. Pour une explication concise du problème voir Stefano Allievi, «Pour une sociologie des conversions : lorsque des Européens deviennent musulmans», Social Compass, 46(3), 1999, p. 285-287. 


\section{1-3 Problématique}

\section{$\underline{\text { Retour à la table des matières }}$}

Nous entendons par identité le rapport avec lequel chaque individu arrive à faire coïncider son être à lui-même, lui-même aux gens qui l'entourent, dans un milieu qu'il définit. ${ }^{21}$ L'identité constitue la fondation de l'être, la base sur laquelle repose sa compréhension du monde. C'est une pensée de l'affirmation de soi qui est réfractaire à d'autres manières d'envisager le rapport au monde. Même s'il est possible pour l'être de se concevoir autrement, le sujet évite le plus souvent cette aventure identitaire de peur de se retrouver altéré. La possibilité d'un «autre » en soi est souvent rejetée parce que ce rapport est vécu comme une menace, une incompréhension, un non-moi.

L'attachement identitaire crée une opposition à la conversion et à l'expression de l'être multiple. La conversion suppose une réflexion sur la façon d'appréhender le monde, ainsi que l'appropriation de différentes variations de soi. Nous pensons que ce phénomène peut être attribuable aux mouvances de l'identité en relation avec l'altérité, mais aussi au développement des événements dans l'extériorité. Autrement dit, le processus par lequel le sens se construit, et qui est constitutif de l'identité, peut varier selon l'évaluation subjective, par l'acteur, de l'évolution de son environnement et de ses relations avec les autres. ${ }^{22}$ L'altération des significations d'une personne est également relative à la prise en compte, par cette dernière, des stimuli en provenance des autres, des choses ou des situations. Ces stimuli peuvent être indépendants d'une volonté connue. On pense ici aux créations de l'expression humaine ou encore à la nature. Dans tous les cas, leur prise en compte ne pourra être considérée que s’ils sont perçus et interprétés par un individu. Et pour que cela se produise, ils devront se trouver dans un cadre significatif pour lui. ${ }^{23}$ C'est cet effort de conceptualisation qui fait accorder les mots avec les faits que nous désignons par le terme, représentation. Il ne suffit donc pas de voir ou de recevoir pour percevoir, il faut simultanément comprendre, saisir la signification du stimulus, ce à quoi il réfère socialement.

D'où la tendance de l'identité comme schème d'interprétation à sécuriser son interprétation du monde, afin d'éluder toute tension émotionnelle désagréable découlant, notamment, d'un manque d'habitudes. ${ }^{24}$ Même si elle s'apprécie aussi dans la

21 Cette définition est inspirée des propos de François Laplantine. François Laplantine, Je, nous et les autres : Être humain au-delà des appartenances, Le Pommier-Fayard, 1999, p. 31-35.

22 Nous allons expliquer ce processus plus en détail un peu plus loin dans ce travail.

23 Jean Stoetzel, La psychologie sociale, Paris, Flammarion, 1963, p. 113-123.

24 François Laplantine, Je, nous et les autres : Être humain au-delà des appartenances, Le Pommier-Fayard, 1999, p. 29. 
différence en relation à l'autre, cette démarche est souvent limitée parce qu'elle n'est pas exigée ou encore trop exigeante. Réitérer son emprise sur le monde pose beaucoup moins de questions que s'ouvrir à d'autres façons de faire. Cela permet d'éviter les pertes de repères inconfortables et l'investissement de soi dans l'apprentissage d'une nouvelle façon de concevoir le monde. C'est pourquoi l'identité préfère, dans certaines situations, s'affirmer et se perpétuer dans l'identique en veillant au maintien de ses représentations. Conséquemment, cette pensée de l'être cherchera le plus souvent à expulser l'altérité en tant que pensée du dehors suspecte, en maintenant la stabilité du caractère et du comportement. Mais subsiste toujours la possibilité qu'elle puisse être altérée par l'étranger. ${ }^{25}$

Cette éventualité qui peut aller jusqu'à se concevoir autrement par l'expérience de la conversion, n'est toutefois pas anodine. Aussi, la menace d'inconfort attribuable à une remise en question de soi risque d'être le plus souvent censurée. La conversion démontre, toutefois, cette faculté chez l'humain de pouvoir exercer sa liberté afin de s'arracher de ses ancrages identitaires.

Si les êtres humains adoptent des comportements selon la nature de leurs appartenances et de leur environnement, ils peuvent, contrairement aux animaux, se distancier de tels attributs en y réfléchissant. Ils sont capables de leur assigner d'autres significations. Aussi considérons-nous avec beaucoup d'attention les comportements dits télésitiques qui découlent d'une telle aptitude. Contrairement aux comportements téléologiques qui correspondent à cette faculté des systèmes vivants de s'inscrire inéluctablement dans le processus de devenir ce qu'ils doivent être, les comportements télésitiques ouvrent la voie à l'acquisition de relations nouvelles avec soi, les autres et l'environnement. Ces derniers rendent compte de la capacité de l'être humain à s'autoanalyser et à se concevoir différemment. ${ }^{26}$ La construction des réalités humaines n'est pas totalement déterminée par l'environnement social et naturel, mais constitue aussi un produit de création qui comporte des contraintes inhérentes aux choix assumés. L'action humaine ne peut donc être entièrement soumise aux forces sociales ou biologiques parce qu'elle démontre aussi une force créatrice qui interagit constamment avec le monde extérieur et qui peut stimuler les êtres humains à s'interpréter et à s'exprimer autrement avec eux-mêmes et avec les autres.

Par conséquent, l'humain qui ne peut saisir objectivement la « réalité » et qui a donc besoin d'ordonner son univers de façon à éviter l'angoisse de l'incompréhensible,

Ibid., p. 41.

Même si les concepts de téléologie et de télésitisme ont été développés par Lee Thayer dans son livre On Communication : Essays in Understanding, Norwood, (New Jersey), Ablex, 1987, p. 71-72, Rousseau, bien avant lui, avait également pressenti avec l'avènement de la modernité, cette ca-pacité de l'humain à se distancier de ses ancrages identitaires. Déjà, à son époque, il écrivait que l'humain, contrairement à l'animal, peut s'émanciper de sa condition parce qu'il peut faire le choix de se perfectionner, ce qu'il désigne par le terme perfectibilité. Luc Ferry, L'Homme-Dieu ou le Sens de la vie, Paris, Grasset, 1996, p. 12-13. Dans le christianisme, on s'y réfère par le terme de rédemption. La notion de résilience y est également associée. 
va rechercher sans cesse l'appui de ceux et celles qui semblent partager ses représentations. Ces représentations ne sont d'ailleurs pas statiques dans le temps et l'espace. Elles sont construites par l'acteur qui est investi dans une relation dialectique avec les autres et qui se transforme à la suite de ses échanges communicationnels. Si on admet un tel postulat, il semble difficile d'attribuer les conséquences des actes de l'acteur social aux seules causes qui lui sont indépendantes. C'est pourquoi nous voulons accorder plus d'attention aux façons qui lui sont données de comprendre ces choses. ${ }^{27}$

Considérant ce que nous venons de dire, le converti n'apparaît donc pas comme un sujet qui subit l'influence des autres, mais comme un acteur qui s'investit avec les limites de sa sensibilité et de sa «compréhension » dans des univers communicationnels signifiants pour lui. Cette capacité d'interpréter le monde qui se note entre autres dans les différences d'habitudes acquises au niveau vestimentaire, intellectuel ou protocolaire n'est pas innée, mais acquise. ${ }^{28}$

L'enfant apprend, dès son plus jeune âge, des façons de se comporter auprès des membres des communautés d'appartenance qui s'imposent à lui comme la famille, les institutions religieuses, d'éducation nationale et de sécurité publique. Les membres de ces groupes primaires dans la socialisation des enfants leur communiquent leurs attentes. Ils les incitent à les imiter en les « séduisant », ou encore font usage de coercition afin de les contraindre à adopter des habitudes conformes à l'environnement socio-culturel dans lequel ils baignent. Ces pressions sociales s'exercent donc par le biais de la coerséduction (coercition-séduction), un processus qui, selon René-Jean Ravault, auteur de ce néologisme, contribue à l'acquisition des habitudes au sein des habitats sociaux. ${ }^{29}$

Cette sensibilisation des enfants contribue à la stabilisation des significations dans l'habitude plutôt que le vagabondage insécurisant pouvant conduire à la nonconformité et à l'incompréhension. C'est pourquoi l'être humain qui fait partie d'une société apprend constamment à comprendre le monde tel qu'exprimé par les autres et s'exprime de façon à ce que les autres le comprennent. ${ }^{30}$

Toutefois, si les êtres humains semblent déterminés par les discours auxquels les soumettent les membres de leurs communautés d'appartenance, ils peuvent dans leur développement vers l'autonomie, choisir de s'investir dans un processus de socialisation secondaire afin d'intégrer à leur gré des communautés que nous avons nommées jusqu'à présent « d'affiliation », mais que nous allons désormais identifier par le terme

27 Lee Thayer, On Communication: Essays in Understanding, (Norwood, New Jersey), Ablex, 1987, p. VIII.

28 Cette phrase fait référence au concept de «l'habitus » développé par Pierre Bourdieu. Nous y reviendrons un peu plus loin dans ce travail.

29 René-Jean Ravault, «Communication dans le monde ; un rêve américain...», In Dictionnaire critique de la communication, sous la dir. de Lucien Sfez, Paris, PUF, 1993, p. 78.

30 Lee Thayer, On Communication : Essays in Understanding, Norwood, (New Jersey), Ablex, 1987, p. VIII. 
épistémiques. La nuance que nous souhaitons souligner ici réside dans le fait que l'intégration à ces communautés va au-delà de l'association, qu'elle suppose aussi le partage d'un paradigme, d'une vision du monde, d'un épistémè, d'une manière d'être propre à un groupe social, à une époque.

En d'autres termes, l'ensemble des connaissances partagées par les membres d'un groupe et que l'on désigne ici par le terme paradigme ou épistémè, constitue bien plus qu'un cadre théorique. Ces termes réfèrent plutôt à des associations d'idées, de généralisations symboliques, de croyances et de valeurs regroupées de façon ordonnée et détaillée et auxquelles adhèrent les membres des groupes d'appartenance ou de communautés épistémiques. ${ }^{31}$

Néanmoins, si le concept d'épistémè renvoie tout comme celui de paradigme à une vision du monde, il réfère aussi plus spécifiquement à un mode de vie, un investissement plus intense de soi, un peu comme ce à quoi réfère la pratique religieuse. Le dogme religieux en tant que paradigme ou épistémè, constitue une matrice de connaissances bien structurée. Plus précisément, il s'agit d'un ensemble complexe de relations logiques constituées, d'une part, de concepts abstraits désignés par des expressions reconnues par les membres d'un groupe religieux, et d'autre part, de présupposés métaphysiques qui fournissent les règles et les valeurs acceptables pour expliquer les problèmes envisagés et les actions à entrevoir. Sa prise en compte signifie aussi que des personnes veuillent s'intégrer à un groupe et en deviennent membres parce qu'elles consentent à partager le sens d'un paradigme ou d'un épistémè particulier. Cela veut aussi dire qu'en plus de souscrire à des croyances théoriques précises qui vont orienter la sélection, l'évaluation et la critique des stimuli qu'elles perçoivent, cette adhésion va aussi guider l'interprétation qu'elles en font. ${ }^{32}$

Toutefois, en ce qui a trait à la conversion religieuse, il ne suffit pas de prendre connaissance de ces matrices de connaissances pour y souscrire, puisqu'il s'agit aussi de s'investir avec d'autres afin de concevoir des significations communes. Cette disposition sous-tend une sensibilité particulière de la part de ceux et celles qui y adhèrent puisque l'acteur social se retrouve dans une situation où il doit s'entendre avec d'autres à propos d'une vision du monde, ce qui signifie qu'il doit négocier et intégrer des discours. L'apprentissage d'un nouveau paradigme ou épistémè risque de s'apparenter à un processus de socialisation secondaire à l'intérieur duquel les échanges seront marqués par l'usage de la persuasion argumentative et des moyens de pression coerséductifs dans le but d'établir un consensus par inclusion des membres et exclusion des autres.

\section{1-3.1 Question et hypothèse centrales}


À la lueur de ces remarques, et considérant, jusqu'à présent, la conversion comme une transformation identitaire, nous ajoutons qu'elle se construit sur le plan communicationnel avec la participation du sujet. Ainsi, initialement, l'acteur social apprend à sécuriser, auprès des membres d'une communauté particulière, son interprétation du monde. Cet apprentissage limite les possibilités qui lui sont données de reconnaître l'ensemble des connaissances. Aussi pouvons-nous nous demander de quelle façon des individus en arrivent à remettre en question leurs allégeances envers leurs communautés d'interprétation puisque cela signifie un détachement par rapport à la façon dont ils s'identifient aux autres. ${ }^{33}$ Et cela sans parler des pressions coerséductives qui s'exercent à l'intérieur des communautés afin que, tous ses membres se conforment aux conventions du groupe. Aussi, c'est en considérant ce problème que nous formulons la question principale de cette étude. Comment est-il possible pour un membre d'une communauté d'interprétation, de remettre en question le discours du groupe auquel il s'identifie? En d'autres termes, pourquoi les rapports de coerséduction des membres de sa communauté, n'arrivent plus à exercer des moyens de persuasion suffisants pour motiver son adhésion au groupe, au point où, il envisage de se convertir?

De prime abord, on peut penser que les pôles de coerséduction, c'est-à-dire les représentations qui font pression sur les membres d'un groupe, se déplacent vers ceux d'un autre groupe parce qu'ils ne s'entendent plus sur l'interprétation des termes de la convention qui les unit. Pour l'instant nous ne pouvons qu'admettre qu'une telle situation comporte une part de risque pour celui qui s'aventure en terrain inconnu ou ne bénéficie pas de l'appui des autres dans son interprétation de la nouveauté ou de ses incompréhensions.

Rappelons que chaque humain, par sa socialisation primaire puis secondaire, développe un potentiel communicationnel propre qu'il met en pratique de façon à exercer un contrôle sur sa vie. Il semble toutefois que cette potentialité soit d'autant plus problématique dans le contexte de la modernité que la conception de l'unité sociale ne procède plus d'une vision hiérarchique basée sur les liens du sang, mais relève plutôt de la négociation des termes d'un contrat entre partis égalitaires. Tant que les pratiques sociales s'exercent selon les conventions d'un ordre social fixe, la possibilité d'exercer une réflexion sur ce sujet se limite à la clarification de la tradition. Avec l'avènement de la modernité, la réflexivité, c'est-à-dire l'acte de se distancier d'un ordre des choses par la réflexion, est devenue une pratique différente. ${ }^{34}$ Elle se distingue de l'introspection, c'est-à-dire de la révision constante de la pensée, sur l'ac-

\footnotetext{
33 Rappelons que les communautés d'interprétation incluent les communautés d'appartenance et les communautés épistémiques.

34 Anthony Giddens, Les conséquences de la modernité, Paris, L'Harmattan, 1994, p. 44-45.
} 
tion, à la lueur des nouvelles informations. Elle consiste plutôt en une réflexion sur la manière de réfléchir. Cette distanciation de l'être dans sa relation à l'ensemble des objets de connaissance participe désormais à la redéfinition constante de l'action des individus et des groupes. Cette propension à relativiser, entre autres en matière de religion des visions du monde distinctes, n'est plus censurée dans le contexte de la modernité par le respect de l'autorité divine. Le sujet conçoit qu'il n'est plus restreint dans sa réflexion sur la religion. La perte de crédibilité subit par le pouvoir religieux expulsé des affaires de l'État, a contribué à l'émergence de pratiques sociales nouvelles. La pratique de la réflexivité s'est exercée à partir de ce moment, différemment. Cette possibilité d'exercer avec plus de liberté la remise en question de soi, contribue à la réévaluation de nos relations envers les autres. Cette pratique suscite des opportunités accrues d'exploration de nouvelles formes d'expression identitaire.

Étant donné ce qui précède, nous énonçons une première hypothèse pour expliquer le processus de conversion identitaire. Nous pensons qu'il survient dans un contexte particulier qui suscite chez l'acteur social une prise de conscience de la relativité de son conditionnement identitaire. Puis, en deuxième hypothèse, nous présumons que, dans le prolongement de cette expérience, l'acteur social est confronté à un problème sérieux d'interprétation associé à la vision du monde qu'il partage, soit avec les membres de son groupe d'appartenance, soit avec ceux de la communauté épistémique élue par choix. Enfin, nous supposons, comme troisième hypothèse, que l'acteur social qui décide d'intégrer une nouvelle communauté d'interprétation, aura développé une prédisposition particulière à l'égard des membres de ce groupe dans le cours de sa socialisation primaire ou secondaire.

Nous estimons que, les expériences de relativisation identitaire, c'est-à-dire celles qui exposent l'illusion des barrières identitaires (associées à l'ethnie, la race, le genre, la religion, etc.), peuvent s'imprégner dans la mémoire. En situation de conflits d'interprétation, elles peuvent être réactualisées dans le temps par l'ensemble des facultés cognitives selon l'interprétation des circonstances. Aussi, en cas de conflit d'interprétation, la mémoire peut concourir à stimuler l'acte réflexif et la communication intrapersonnelle. Le pouvoir de distanciation face à soi, peut s'exercer de façon à stimuler la reconnaissance des attachements identitaires qui portent à confusion, suscitent des tensions. La pratique réflexive peut faciliter l'identification des sources de conflits en soi, en relation aux autres. Afin d'atténuer cette état de tension désagréable qui expose et remet en question la façon dont est appréhendé le monde, la personne risque de faire preuve d'une plus grande ouverture face à d'autres opportunités d'association avantageuses, surtout si elles sont associées à une communauté qui exerce un attrait dans l'expérience de vie de celle-ci. L'adoption d'une vision nouvelle des choses peut alors être considérée comme une solution plausible. Mais, l'identification à une nouvelle communauté de pensée exige, en plus, que la réalisation des projets du nouveau groupe apparaisse plus conforme, moralement, aux croyances de la personne qui se remet en question. Nous estimons que le manque de correspondance entre l'idéal, source initiale de rassemblement, et sa réalisation prati- 
que, peut devenir une source de conflits suffisamment grande pour entraîner l'ouverture à des univers communicationnels autres.

Enfin, l'acteur social qui se remet en question n'effectue pas ses recherches en tout sens. Malgré la diversité des points de vue offerts dans le contexte de la modernité, l'être humain, ne pouvant faire abstraction de son identité, sélectionne l'information en provenance des autres. Cette sensibilité à l'égard de leurs propos ne signifie pas que les significations des uns correspondent à celles des autres. Elle consiste plutôt en une disposition de l'esprit qui s'acquiert dans le cours de la socialisation primaire des individus. Elle se développe, également, lorsque les individus font preuve de «télésitisme » en se distanciant de leur conception des choses. Elle peut servir à remédier à la perte des avantages associés à l'intégration d'un groupe auquel une personne renonce. Ainsi, si l'identification est orientée de façon à se reproduire au sein des communautés d'interprétation, elle peut aussi s'émanciper dans l'altérité au contact d'un réseau de relations secondaires. La rencontre avec l'autre, qui peut sembler de prime abord si différent, peut être intériorisée comme un idéal à atteindre. Cette rencontre peut aussi devenir révélatrice de l'ipséité, c'est-à-dire de l'Autre en soi. L'être humain peut accéder à d'autres visions du monde et ainsi exercer différemment sa perception de lui-même et de l'extériorité. La relation avec l'autre à l'extérieur de soi, est alors appréciée non pas uniquement dans la différence, mais aussi dans la prise de conscience des similitudes inhérentes à la condition humaine. Cette considération crée une ouverture à la singularité de l'autre et à l'appréciation de la diversité des points de vue. La transgression de l'illusion identitaire incite à l'élaboration de relations humaines moins codées, offrant plus d'espace pour innover et faire preuve de créativité. La monotonie du quotidien cède alors à l'aventure humaine.

\section{1-3.2 Hypothèses sectorielles}

\section{$\underline{\text { Retour à la table des matières }}$}

Si la possibilité d'entrevoir une vision autre du monde existe, cela n'implique pas qu'elle soit nécessairement adoptée. Dans diverses études, des chercheurs ont tenté de démontrer les facteurs qui pourraient expliquer comment les personnes adhèrent au point de vue des autres. Les recherches menées en communication par des chercheurs de « l'École de Columbia » sur la dynamique des influences interpersonnelles dans les groupes primaires et secondaires ont démontré que, l'influence d'un discours religieux ne peut être attribuable uniquement à son exposition. Nous supposons plutôt, que le 
pouvoir de persuasion d'un discours est activé lorsqu'il y a reconnaissance par l'acteur social, des trois éléments suivants : ${ }^{35}$

1) une autorité compétente dans l'évaluation et la négociation de nouvelles pratiques sociales au sein d'un groupe donné (ce que l'on sait),

2) la personnification par ce leader de certaines valeurs particulières (qui l'on est),

3) la position sociale stratégique que ce dernier exerce, c'est-à-dire qui il connaît à l'intérieur et à l'extérieur du groupe. ${ }^{36}$

Ces données appliquées à Roger Garaudy, c'est-à-dire à un intellectuel renommé et reconnu dans les communautés catholiques et marxistes convertit à l'islam, permettent de poser les hypothèses sectorielles suivantes :

$1^{\circ}$ Nous nous demandons sur quelle base un tel leader d'opinion qui évolue dans un environnement où il est continuellement en contact avec de nombreuses personnalités reconnues, va accepter de se laisser persuader par l'une d'entre elles?

Nous présumons que des leaders d'opinion faisant preuve de talent équivalent, se reconnaissent selon leur capacité à communiquer et à se référer à des valeurs similaires.

$2^{\circ}$ Ensuite, nous voulons comprendre comment une telle personne, investie autant dans une communauté d'appartenance (sur le plan religieux) que dans une communauté épistémique (le Parti communiste français), en vient à entrer en conflit avec les deux pour ensuite en intégrer une troisième (l'islam)?

Nous pensons que le discours de certains groupes s'ajuste mal, pour certains adhérents, aux situations nouvelles qui se présentent. D'autres organisations qui offrent des possibilités multiples d'expression exercent alors plus d'attrait.

$3^{\circ}$ Enfin, nous recherchons quelles sont les conséquences pratiques d'une conversion? Nous considérons que si le converti a besoin de reconnaître le

35 Comme nous l'avons déjà mentionné, les recherches auxquelles nous faisons allusion sont celles de Merton sur l'influence interpersonnelle à Rovere ; de Katz et Lazarsfeld sur la formation des décisions dans différents secteurs d'activité ; de Berelson, Lazarsfeld et MacPhee sur l'influence du vote lors de la campagne électorale de 1958 ; ainsi que celle de Coleman, Katz et Menzel sur la diffusion d'un nouveau remède par les médecins. Elihu Katz, «Les deux étages de la communication (The two-step flow of communication)», In Sociologie de l'information, sous la dir. de F. Balle et J. G. Padioleau, Paris, Larousse, 1973, p. 287.

36 Ibid., p. 299. 
leader d'opinion pour adhérer à ses propos, le leader d'opinion, en retour, devra aussi reconnaître le converti.

Nous supposons donc que l'acteur social participe à la construction de son expérience de conversion selon la relation communicationnelle qu'il entretient avec lui-même mais aussi en fonction des avantages que lui procure sa relation avec les autres.

En résumé, on peut dire que l'appréhension de la « réalité » par les acteurs sociaux découle des constructions communicationnelles qu'ils élaborent en fonction de la relation dialectique qu'ils entretiennent avec eux-mêmes et leurs communautés d'interprétation, dans un contexte donné. Puisque l'acteur tend à préserver ses significations, la conversion religieuse démontre les facultés télésitiques de l'être l'humain. Celles-ci lui permettent de se distancier de lui-même afin d'expérimenter avec d'autres de nouveaux échanges qui lui paraissent plus significatifs.

Nous avons également mentionné, que le processus de conversion religieuse a souvent été perçu, sous l'angle d'analyses voulant que des circonstances extérieures contraignent le sujet à adopter d'autres comportements, ou encore que sa personnalité le prédispose à vivre ce genre d'expérience. Très peu de recherches ont accordé de l'importance au processus de réception active chez l'être humain qui s'investit dans l'expérience de conversion religieuse. ${ }^{37}$

Mis à part André Billette qui a reconnu un tel phénomène. Après avoir voulu recueillir des informations supplémentaires auprès des convertis qu'il avait interviewés deux ans auparavant dans le contexte de sa recherche, il a constaté des divergences frappantes par rapport aux récits initiaux. Cette constatation démontre que la dimension subjective de la construction de l'expérience de conversion religieuse s'élabore par le discours au fur et à mesure que l'acteur attribue des significations à son vécu. André Billette, Récits et réalités d'une conversion, Montréal, Presses de l'Université de Montréal, 1975, pp. 13-15. 


\title{
Chapitre II
}

\section{Cadre de référence théorique}

\author{
« Nous construisons ce que nous regardons à mesure que ce \\ que nous regardons nous constitue, nous affecte et finit par \\ nous transformer. » 38
}

\section{2-1 Aperçu de la recherche sur les conversions à l'islam dans le contexte européen}

Comme nous l'avons laissé entendre dans l'énonciation de nos hypothèses, nous allons analyser comment se construisent, par la communication intrapersonnelle et interpersonnelle, les problèmes d'interprétation graves que peut vivre un acteur social à l'intérieur de sa communauté d'appartenance ou épistémique et qui vont l'inciter à vouloir se convertir.

Mais avant d'expliquer avec quelle perspective d'analyse nous souhaitons aborder notre tâche, nous voulons passer brièvement en revue, les idées directrices des études les plus prometteuses, en matière de conversion religieuse et plus spécialement celles portant sur les conversions à l'islam dans le contexte européen. Ceci nous permettra de mieux situer notre démarche par rapport à ce qui a déjà été proposé sur ce sujet. 
Car il semble que les chercheurs qui se sont efforcés d'expliquer le phénomène à partir d'un cadre d'analyse spécifique, se rendent compte maintenant, des limites inhérentes à une telle façon de procéder. Aussi envisagent-ils, pour la plupart, un champ d'exploration plus large tenant compte de facteurs empruntés à diverses disciplines. ${ }^{39}$

$1^{\circ}$ Ali Köse attribue les conversions à l'islam au relâchement des normes morales entraîné par la laïcisation des sociétés occidentales. Pour elle, ce processus a eu pour conséquence d'engendrer une perte de repères et de sens. ${ }^{40}$

$2^{\circ}$ Madeleine Sultán spécifie que si, «traditionnellement la conversion signifie la transition d'une foi à une autre », ce sont aujourd'hui «[...] les gens qui n'ont pas ou très peu d'expérience religieuse qui deviennent adeptes. » ${ }^{41}$

$3^{\text {o }}$ Monika Wohlrab-Sahr démontre que les conversions constituent des solutions pour des personnes aux prises avec des problèmes d'intégration et de désintégration du contexte social dans lequel elles sont insérées. ${ }^{42}$

$4^{\circ}$ Sarah Daynes explique que la conversion constitue une quête identitaire dont la signification est multiple. «Se convertir c'est en quelque sorte devenir un autre : changer de comportement, adopter un mode de pensée nouveau, quitter un groupe pour en intégrer un autre [...]. » 43

$5^{\circ}$ Mercedes García-Arenal souligne que si, par le passé, les conversions marquaient le passage d'une minorité vers une majorité, elles procèdent en sens contraire aujourd'hui. Par exemple, des personnes issues d'une société majoritairement catholique vont se convertir à la religion d'un petit groupe d'immigrants. ${ }^{44}$ Ces conversions s'établissent sur la base de l'identification à l'étranger, l'autre, et sont donc porteuses d'enjeux intercommunautaires. ${ }^{45}$

$6^{\mathrm{O}}$ Thomas Luckmann ajoute que le processus de modernisation, caractérisée, entre autres, par la spécialisation et la différenciation fonctionnelle des institutions économiques, politiques et religieuses a eu pour conséquence de restreindre le poids

39 Les mots en italique dans les explications qui suivent servent à attirer l'attention du lecteur sur des facteurs qui nous semblent importants dans l'explication de la problématique de la conversion.

$40 \quad$ Ali Köse, « The Journey from the Secular to the Sacred : Experiences of Native British Converts to Islam », Social Compass, 46(3), 1999 pp. 301-312.

41 Madeleine Sultán, «Choosing Islam: a Study of Swedish Converts», Social Compass, 46(3), 1999, p. 326.

42 Monika Wohlrab-Sahr, «Conversion to Islam: Between Syncretism and Symbolic Battle», Social Compass, 46(3), 1999, p. 352.

43 Sarah Daynes, «Processus de conversion et modes d'identification à l'islam : l'exemple de la France te des Etats-Unis », Social Compass, 46(3), 1999, pp. 313-314.

44 Mercedes García-Arenal, «Les conversions d'Européens à l'Islam dans l'histoire : esquisse générale», Social Compass, 46(3), 1999, p. 273.

45 Ibid., p. 279-281. 
des normes et coutumes imposées par la religion. ${ }^{46}$ Cela a augmenté la liberté de contacts entre les groupes et ouvert la possibilité d'un vagabondage idéologique et spirituel. La possibilité de choisir entre plusieurs représentations collectives suppose qu'une compréhension plus complète du phénomène de conversion passe aussi par l'étude du canon adopté. Cela nécessite de prendre en considération les interactions communicationnelles au sein des institutions religieuses qui contribuent à façonner son interprétation. ${ }^{47}$

$7^{\circ}$ Stefano Allevi apporte une distinction intéressante qui présente, de façon succincte, une typologie des conversions à l'islam. Cette classification permet de regrouper et d'expliquer une série de facteurs qui y contribuent. ${ }^{8}$ Dans le cas où certaines croyances pousseraient à entreprendre des actions spécifiques, il parle de conversion rationnelle ou intellectuelle, tandis que lorsque des considérations pratiques incitent la recherche d'une croyance, il les perçoit comme des conversions relationnelles.

$8^{\circ}$ Lofland et Skonovd considérant l'importance de la relation qu'entretient le converti avec le groupe religieux, ont accordé beaucoup d'importance aux pressions sociales s'exerçant sur les convertis. ${ }^{49}$

$9^{\circ}$ Enfin, Lewis Rambo formule l'idée qui, pour nous, incorpore toutes les autres. Il avance que le processus de «[...] conversion est activement construit par les groupes religieux ainsi que par les vœux, les attentes et les aspirations du converti ou du converti potentiel. » Aussi suggère-t-il que la « [...] conversion constitue ce qu'un groupe de personnes en disent. » Le processus de conversion devient ainsi « [...] un produit des interactions entre le converti et ses aspirations, ses besoins, et ses orientations, la nature du groupe auquel il se convertit et la matrice sociale particulière à l'intérieur de laquelle prennent place ces processus. » 50

Lorsque nous cherchons à regrouper l'ensemble des idées qui orientent ces recherches, nous remarquons que les auteurs accordent beaucoup d'importance à la modification du contexte social qu'entraîne la modernité. Ils font allusion à la séparation de l'Église et de l'État en Occident, qui a affaibli l'emprise de l'institution religieuse sur la société. L'Église, en étant désormais contrainte à une autorité limitée ne pouvait

46 Thomas Luckmann, «The Religious Situation in Europe : the Background to Contemporary Conversions», Social Compass, 46(3), 1999, pp. 251-258.

Selon le Petit Robert 1, dictionnaire de la langue française, le terme canon réfère à la loi ecclésiastique ainsi qu'à l'ensemble des livres admis comme divinement inspirés.

On peut consulter l'article de Stefano Allievi, «Pour une sociologie des conversions : lorsque des Européens deviennent musulmans», Social Compass, 46(3), 1999, pp. 289-294., mais le lecteur aura beaucoup plus de précisions sur le sujet dans son livre Les convertis à l'islam : Les nouveaux musulmans d'Europe, Paris, L'Harmattan, 1998.

49 John Lofland et Norman Skonovd, «Conversion Motifs», Journal for the Scientific Study of Religion, 1981, 20(4), p. 373.

50 Lewis R. Rambo, Understanding Religious Conversion, New Haven and London, Yale University Press, 1993, p. 7. 
plus imposer aux populations ses normes et, par conséquent, contrôler l'ensemble des actions et des significations sociales. Cette situation a contribué à l'émergence d'une panoplie de groupes défendant des points de vue idéologiques et spirituels variés. Les motivations des individus sont alors apparues plus clairement puisque, dès lors, existait la possibilité d'explorer de nouvelles avenues, d'errer plus librement et, conséquemment, de questionner la soumission sociale aux significations religieuses.

\section{2-2 Distribution sociale de la connaissance}

Retour à la table des matières

Certes, cette liberté d'exploration facilite l'expérience individuelle, mais on ne peut ignorer le fait que la religion, comme la plupart des systèmes idéologiques qui apportent des solutions aux problèmes de la vie en groupe, constitue aussi une expérience collective. $\mathrm{Si}$, à première vue, la « réalité » vécue par chacun apparaît comme un ensemble de connaissances déterminées qui vont de soi, elles expriment aussi des regroupements d'idées qui s'élaborent en fonction des situations historiques et des contextes sociaux. Par conséquent, nous accordons de l'importance à la distribution sociale de la connaissance. Ce traitement sociologique des objets de connaissance n'aborde pas uniquement leur validité empirique, mais considère également les « [...] processus par lesquels tout corps de « connaissance » en vient à être socialement établi en tant que « réalité ». » 51

Qu'il s'agisse de représentations, de paradigmes ou d'épistémè, ces connaissances sont liées à l'environnement d'où elles émergent, parce que l'on suppose que la personne crée son propre monde. On ne présume pas de l'acquis des acteurs en ce qui a trait à leur conception de l'ordre social ou des valeurs sociales, mais on cherche plutôt à les découvrir chez la personne en situation. Cette vision du monde, désignée par le terme interactionnisme, comporte trois idées primordiales. ${ }^{52}$

En premier lieu, l'organisation sociale, institutionnelle ou autre, bien qu'elle soit préexistante, n'est pas déterminante sur l'activité humaine ; au contraire, elle résulte plutôt de la rencontre entre les groupes, de la résolution ou non des conflits, des négociations, des processus d'apprentissage et de connaissance. Il s'agit donc d'une position anti-déterministe.

51 Peter Berger et Thomas Luckmann, La construction sociale de la réalité, Paris, Méridiens Klincksieck, 1996, p. 10.

52 Anselm Strauss, La trame de la négociation: Sociologie qualitative et interactionnisme, Isabelle Baszanger (compilatrice), Paris, l'Harmattan, 1992, p. 14. 
Ensuite, les ressources de l'activité humaine (compétences cognitives, règles, catégories, positions sociales...), s'élaborent selon les représentations de la « réalité » qui sont conformes aux perceptions ou opinions de plusieurs personnes. Tout en étudiant les processus de coordination des activités ainsi que les interactions dans leur contexte, on peut accorder une attention particulière au caractère symbolique de l'action sociale telle que manifestée dans l'utilisation du langage.

Enfin, les multiples facettes de la relation entre action et réflexion découlent de l'activité cognitive. Par conséquent, elles sont influencées par la créativité, l'intuition et le caractère réflexif de l'humain, c'est-à-dire par sa capacité à se distancier de luimême. On ne peut donc réduire l'action humaine à une soumission aux forces sociales. Il existe également une intelligence créatrice qui interagit constamment avec le monde environnant et qui peut inciter les êtres humains à se dépasser. Il s'agit de décrire le processus interprétatif dans lequel chacun s'investit avec lui-même, ainsi que l'influence de l'autre sur soi et de soi sur l'autre. L'être humain construit sa vie en fonction de la relation qu'il entretient avec lui-même, les autres, l'environnement dans lequel il évolue, bref en regard de sa relation au monde en général.

Évidemment la question de la construction des réalités pose le problème de l'impossibilité de la connaissance à saisir la « réalité » même. Bien des penseurs ont tenté d'y répondre. Ils ont cherché à esquisser l'interrelation des différents éléments expliquant la relativité des points de vue. Nous souhaitons faire un survol rapide de leur questionnement pour mieux comprendre en quoi consiste la sociologie de la connaissance, et ainsi mieux saisir les enjeux relatifs à l'objet de ce mémoire.

Le questionnement sur les fondements sociaux des visions du monde et des valeurs remonte à l'Antiquité. Mais c'est Max Scheler qui va introduire, dans les années 1920, une perspective philosophique sur cette problématique. Sa réflexion va stimuler l'essor de la sociologie de la connaissance. On attribue toutefois à Marx la position fondamentale de cette nouvelle discipline selon laquelle : «[...] la conscience de l'homme est déterminée par son être social. » ${ }^{53}$ Cette conscience n'est pas étrangère aux concepts d'idéologie (vision particulière du monde qui permette de l'interpréter et défendre des intérêts sociaux), de fausse conscience (ou d'aliénation), d'infrastucture (qui correspond à l'activité humaine) et de superstructure (qui est le monde produit par cette activité).

Nietzsche, en substituant la recherche du vrai pour celle du sens, va également stimuler de nouvelles perspectives d'analyse sur le sujet. Il va poser le problème de l'origine des valeurs et de leur utilisation comme instrument de combat pour la survie et le pouvoir.

53 Peter Berger et Thomas Luckmann, La construction sociale de la réalité, Paris, Méridiens Klincksieck, 1996, p. 13. 
La contribution de William Dilthey n'est pas non plus négligeable ; il démontrera l'incapacité de la pensée humaine à comprendre une situation sociale en dehors de son contexte historique. Mais l'ensemble des variables évoquées - l'aliénation, la réalité comme produit des activités humaines, la survie, le pouvoir, l'historicité - si elles offrent une connaissance théorique de ce qui semble «réel », elles ne peuvent vraiment définir les fondements logiques de la pensée. Il semble difficile de transcender les problèmes que soulève le relativisme et que la sociologie de la connaissance interroge.

Toutefois, Scheler va analyser ce qui détermine, dans la société, la réglementation de la connaissance humaine. ${ }^{54}$ Il en ressortira que la connaissance humaine de la société découle de l'expérience individuelle qui lui en fournit les règles ainsi que le sens. Cette vision du monde, particulière à l'individu inséré dans un contexte sociohistorique défini, réfère au concept de Weltanschauung.

Nombre de chercheurs vont pousser leurs investigations sur d'autres pistes. Mais on voit bien, que la sociologie de la connaissance cherche à éclaircir le processus d'acquisition des connaissances d'un point de vue épistémologique. Elle questionne la façon dont nous exerçons notre jugement par rapport à un objet d'analyse et comment nous nous positionnons, étant donné que nos investigations sont toujours empreintes de notre subjectivité, qui est elle-même conditionnée par nos références sociales et scientifiques.

Bien que ce questionnement soit présent au sein de l'ensemble des sciences sociales, il s'agit, plus spécifiquement, de concevoir comment s'effectue le « commerce » des idées, la construction des Weltanschauungen, comment chacun participe à sa connaissance. Selon cette perspective d'analyse, une attention particulière est portée sur ce que les gens connaissent comme « réalité » au quotidien, en d'autres termes le sens commun, beaucoup plus que sur les idées d'ordre théorique. Plus concrètement, la distribution de la pensée du sens commun s'observe à l'intérieur de cadres socioculturels et historiques précis ainsi que par sa pertinence au niveau de l'environnement social. Par exemple, la conversion à l'islam d'un Américain vivant aux États-Unis risque d'être beaucoup plus désapprouvée aujourd'hui qu'auparavant, tandis que cette même conversion, ailleurs dans le monde, pourrait susciter une tout autre réaction. Il y a donc une distribution sociale de la connaissance et l'articulation de ce processus devient la Weltanschauung de chacun. Si nous acceptons que la société, en tant que dimension artificielle objective, exprime un sens subjectif, alors il faut peut-être se poser la question de savoir comment se transige cette activité humaine des points de vue objectif et subjectif. 


\section{2-3 Sur le sens du processus de réception}

$\underline{\text { Retour à la table des matières }}$

Nous pensons que cette négociation s'élabore par l'intermédiaire de la communication et du sens attribué aux échanges. Il est important de noter que l'assignation d'un sens ne constitue pas le fruit d'un hasard, mais est fonction de la perception des situations dans un contexte donné. Nous ne percevons de la « réalité » que ce que nous y mettons nous-mêmes. En d'autres termes, les stimuli que nous appréhendons ne font sens pour nous que dans la mesure où ils peuvent être décodés par notre esprit selon nos projets en cours ainsi que par notre expérience antérieure, sensorielle et conceptuelle. ${ }^{55}$ Deux personnes, côte à côte, peuvent être témoins d'un même accident et en donner deux versions différentes sans malhonnêteté de leur part. ${ }^{56} \mathrm{Si}$, à partir d'un certain âge, les enfants perçoivent l'identité de volume entre des récipients étroits et larges, ils acquièrent aussi la perception que statistiquement la plupart des objets situés en hauteur sont plus gros que ceux qui sont situés à leur niveau. Ce n'est qu'à la suite d'expérimentations qu'ils constateront l'exception à cette règle, et qu'ils apprendront, par coerséduction, une stratégie perceptive conforme à la vision éprouvée par les membres de leurs communautés d'appartenance. La perception des stimuli sensoriels ne constitue donc pas le miroir de l'événement auquel nous sommes confrontés. ${ }^{57}$ Bien qu'elle passe par les sens, c'est en fait par une circulation de signes véhiculés en nous par un système complexe de nerfs et de processus cérébraux biochimico-électriques, que se formera une représentation propre à la grille de décodage de notre esprit. On comprend donc qu'il s'agit d'une opération de traduction et non du reflet d'un fait extérieur sur la plaque photographique que constituerait l'esprit.

Il y a toutefois des nuances fort importantes à apporter à ces propos, nuances qui, créent d'ailleurs beaucoup de dissension au sein des chercheurs en communication. Ils sont divisés en deux camps. Ils n'adhèrent pas à une même vision du monde quant à l'interprétation du processus de décodage, processus qu'il nous est impossible de

Thomas S. Kuhn, La structure des révolutions scientifiques, Chicago, Flammarion, 1983, p. 160. Edgar Morin parle sur ce sujet de « la composante hallucinatoire de la perception qui est déterminée non par un facteur « irrationnel » (affectif, magique), mais par un principe de rationalité ». Mais il nous met aussi en garde contre le traumatisme et l'émotion violente qui peuvent l'induire irrationnellement. Edgar Morin, Pour sortir du vingtième siècle, Paris, Fernand Nathan, 1981, pp. 18-21.

Si nous avons parlé de stimuli sensoriels, c'est parce que cette expression nous paraissait rendre neutre le processus de décodage. Cela permettait ainsi d'éviter la mention du terme «message» qui infère dans l'esprit de plusieurs une connotation d'impact parce que l'intention de l'émetteur est alors prise en considération. 
voir parce que situé dans «la boîte noire» de l'esprit. Pour les partisans de l'approche dite "victimisante », l'esprit des individus, comme un appareil photo, se laisserait imprégner par des stimuli sensoriels comme la lumière affecte le négatif de la pellicule, ce qui leur ferait adopter, par l'intermédiaire de la pratique discursive du langage, des significations socialement partagées. ${ }^{58}$

Mais cette façon de conceptualiser l'attribution du sens pose problème. En effet, elle limite cette opération à l'immédiateté, en plus de la restreindre à un décodage convenable, voire littéral et selon des conventions de signes et de langage apparemment universelles. Cette conceptualisation est conditionnée culturellement en Occident par les religions du Livre et par toutes les disciplines d'enseignement où l'on prétend à une vérité d'adéquation plutôt qu'à une vérité de dévoilement. Elle sous-entend que certaines personnes possèdent les qualités pour s'approprier le juste sens ou encore que les artefacts culturels qu'ils produisent reflètent ces qualités. 59

D'autres chercheurs inversent cette perspective d'analyse en démocratisant cette proposition élitiste. Ils pensent plutôt que tous les humains participent à la construction du sens par leurs interactions communicationnelles avec les personnes de leur environnement culturel physique et mental. En accord avec la métaphore de Max Weber, ils croient également que l'humain, à l'image de l'araignée, tisse la toile de sa culture (ou la Weltanschauung) qui lui permet d'appréhender le monde et lui donner sens. 60 En communication, l'imprégnation du sens à partir de l'extériorité fait référence au paradigme photo tandis que le processus de réception et d'interprétation renvoie à celui du radar. Ce dernier réfère à une vision du monde où les personnes ne sont pas destinataires de messages, c'est-à-dire que le sens des représentations partagées ne comporte en soi aucune intention universellement acceptée, et que, par conséquent, les personnes sont simplement exposées à un signe dont elles construisent le sens selon leur propre grille de décodage. Cette grille s'apparente à la carte de fond d'un écran radar qui est balayée en surface. Ainsi le récepteur, tout comme un radar, balaierait l'extériorité par ses sens et la percevrait selon sa sensibilité.

Cette réceptivité au monde extérieur serait alors interprétée en fonction des normes, des valeurs et des croyances qu'il aurait acquises par coerséduction au sein des groupes auxquels il s'identifie ; tout d'abord par obligation, aux communautés d'appartenance, puis, auprès des communautés épistémiques choisies.

58 René-Jean Ravault, «Développement durable, communication et réception active», In Communication et développement international, sous la dir. de Thérèse Paquet-Sévigny, Sainte-Foy, (Qué.), Presses de l'Université du Québec, 1996, p. 67-68.

59 Le terme artéfact culturel fait ici référence à la notion de produits culturels, comme les livres, films, émissions de télévision, etc. La distinction entre les deux résident dans le fait que l'artéfact culturel ne sous-entend pas véhiculer un sens particulier, le sens étant conçu par les personnes et non contenu dans les choses.

60 René-Jean Ravault, «Développement durable, communication et réception active», In Communication et développement international, sous la dir. de Thérèse Paquet-Sévigny, Sainte-Foy, (Qué.), Presses de l’Université du Québec, 1996, p. 69. 
Dans un cas comme dans l'autre, le récepteur y expérimente une culture qui lui permettra d'interpréter le monde tout en s'interprétant lui-même. Cette relation dialectique implique des compétences communicationnelles propres à l'identité. La capacité des individus à entrer en interaction se constitue lors du processus de socialisation en bas âge. Cet apprentissage est également sujet à l'influence du contexte social dans lequel vont se situer les relations au sein des groupes. Nous allons expliquer plus amplement l'importance de ses deux facteurs qui nous aideront à comprendre, d'une part, l'instauration de la cartographie de fond de l'écran radar, par imposition photographique, et d'autre part, le contexte participant à moduler ce système. Nos propos peuvent paraître à première vue contradictoires parce que nous semblons reconnaître ici le paradigme photo. En fait, l'un et l'autre sont à l'œuvre et s'entrechoquent pour se compléter.

Il nous apparaît donc judicieux d'expliquer, dans un premier temps, comment s'effectue le processus de socialisation primaire ainsi que la construction des représentations à l'intérieur des communautés d'appartenance pour mieux saisir par la suite comment une personne en arrive à remettre en question le sens de cette « réalité » partagée et à s'investir éventuellement dans un processus de socialisation secondaire entraînant sa conversion.

\section{2-3.1 La socialisation primaire}

\section{$\underline{\text { Retour à la table des matières }}$}

Pour l'enfant, la compréhension sociale de la « réalité » et de la «connaissance » va se constituer en bas âge et varier selon l'expérience acquise sur le plan social et culturel. Pour Pierre Bourdieu, cette opération est fonction du rapport entre les positions sociales, l'habitus, et les prises de position. ${ }^{61}$ En d'autres termes, la position que les individus occupent dans l'espace social selon leur capital économique et culturel, contribue au façonnement de leur habitus, c'est-à-dire des dispositions qui influencent leurs prises de décisions.

Ce processus explique non seulement les nombreuses variations de perception mais aussi l'adoption de pratiques très différentes quant à la façon de manger, de choisir et de pratiquer un sport, de sélectionner des biens, ainsi que d'exprimer ses opinions. Ces pratiques variées constituent des codes symboliques identifiables, qui expriment un véritable langage. 
Cette démarcation des uns par rapport aux autres, renferme donc l'idée d'une construction des différences, d'une façon de faire qui nous distingue les uns des autres, d'un groupe à l'autre. Aussi «[...] la position occupée dans l'espace social, c'est-à-dire dans la structure de la distribution des différentes espèces de capital, qui sont aussi des armes, commande les représentations de cet espace et les prises de position dans les luttes pour le conserver ou le transformer. » ${ }^{62}$ En d'autres termes, les représentations propres à une personne sont conformes à son capital social et culturel et cela détermine en quelque sorte comment elle se conçoit dans ses relations sur le plan social et comment elle entend les préserver ou les modifier.

Cette matrice des relations qu'un individu entretient auprès des autres, favorise la persistance d'un lien social qui ne pourrait perdurer dans le cas où les individus ne verraient qu'à la satisfaction immédiate de leurs besoins et pulsions. ${ }^{63}$ La stabilité sociale passe par la répression d'Éros, ennemi de la répétition qui se manifeste dans le caractère fluctuant de l'humain, dans ces hauts et ces bas, dans l'avant et l'après de l'acte sexuel, dans les charges et décharges affectives. La société nie l'existence des pulsions et, en procédant de la sorte, elle prescrit comment les individus vont s'associer : « avec sa cousine mais non avec sa fille, avec quelqu'un de la même religion ou classe sociale et non d'une autre religion ou d'une classe sociale différente. » ${ }^{64}$ L'intériorisation des interdits sociaux est ainsi incorporée dans la vie psychique. L'attachement affectif qui incite le sujet concerné à s'identifier à l'être aimé, peut être intériorisé de façon à ce qu'il lui permette de se dominer et de dominer la relation à autrui. L'identification, qu'elle soit libidinale comme Freud l'entrevoyait, ou non, correspond à la découverte de traits communs avec une autre personne. Plus l'univers commun est grand, plus l'identification sera complète et engendrera un sentiment d'attachement. L'identification contribue à la reproduction de modèles et se traduit donc par l'acte d'imiter. "L'imitation, écrit le psychologue russe Bechterew, qui a été si bien mise en lumière par Tarde, est la conséquence naturelle de la reproduction d'un acte [...] laissant dans les voies nerveuses des traces qui facilitent la répétition et incitent à le répéter. » 65

Aussi, selon Ralph Linton, les comportements explicites des individus ne constituent pas des réponses instinctives à leurs besoins, mais démontrent l'assimilation de l'expérience en considération des conditions imposées par l'environnement. ${ }^{66}$

62 Ibid., p. 28.

63 Serge Moscovici, L'âge des foules, Bruxelles, Complexe, 1981, pp. 335-337.

64 Ibid., p. 336.

65 Cette citation, dont la référence apparaît plus haut, est tirée du livre L'âge des foules de Serge Moscovici à la page 338. Mais l'originale provient de W. Bechterew, La réflexologie collective, Neuchâtel et Paris, Delachaux et Niestlé, 1957, p. 167.

66 «Les individus qui composent une société [...] sont classés et organisés en plusieurs systèmes simultanés. » Ainsi chaque membre de la société se positionne dans le système âge-sexe ou dans les catégories de prestige, et peut appartenir à une unité familiale ou groupe associatif ; « [...] il occupe de ce fait une place parfaitement définie dans le système que ces groupes constituent ». "Chacun de ces systèmes remplit sa fonction propre dans le rapport de l'individu avec sa culture, et l'individu occupe une place dans chacun d'eux ». Puisque les systèmes persistent au- 
L'ensemble de ses réponses n'est pas uniforme, mais la familiarité des situations engendre des habitudes qui préservent l'énergie nerveuse en diminuant le niveau de tension émotionnelle. ${ }^{67}$ L'investissement de soi dans l'adoption de nouveaux comportements est donc fonction de leur efficacité à faire face à une situation nouvelle. Lorsqu'un individu n'a aucune référence de comportement dans un contexte donné, sa réponse initiale consistera à se référer à son expérience passée, ou à adopter des modèles de comportements nouveaux, par imitation. Ainsi, parmi les fluctuations de l'inconnu qui engendrent l'insécurité, va apparaître l'uniformité d'un ordre qu'il reconnaît comme sien. ${ }^{68}$ Lorsque qu'un individu se retrouve dans un salon en présence d'un groupe de personnes inconnues, il aura tendance à rechercher celles dont l'âge, la profession, les opinions lui paraissent familiers. Cette simulation ne constitue en fait qu'un moyen de dissimulation contre la peur d'être exclu par autrui et qui est parfois justifiée, dans la mesure où le paraître social peut exacerber le narcissisme de certaines personnes voulant être reconnues dans un groupe, aux dépens des autres.

C'est pourquoi un groupe affectif et restreint comme celui de la famille va tendre à faire disparaître cette indétermination afin de sécuriser sa progéniture. Toutefois, si le jeune enfant cherche, dans un premier temps, à imiter ses parents jusqu'à vouloir se fondre en eux, le renoncement à ces sentiments amoureux troubles au profit d'une intériorisation du modèle parental, va lui permettre de gagner son autonomie. ${ }^{69}$ C'est ainsi que l'enfant se construit en relation au monde extérieur, se socialise en intégrant à son moi, à sa personnalité, des jugements et des interdits tout en continuant d'intégrer par imitation, l'influence que ses parents exercent sur lui. En termes simples, l'enfant se socialise en adoptant les normes de sa famille et en imitant ses parents.

\section{2-3.2 Formation de l'identité par " coerséduction »}

$\underline{\text { Retour à la table des matières }}$

Si pour Gabriel Tarde, l'amour filial exerce un pouvoir de « séduction » qui motive l'enfant, pour Émile Durkheim ce sont plutôt les rapports de coercition qui s'exercent tout d'abord au sein de la famille, puis dans la communauté, par le biais

delà de la vie des individus, Linton définit le statut comme la place qu'occupe un individu donné, dans un système donné, à un moment donné. Le rôle ou le comportement explicite d'un statut, c'est-à-dire ce que l'individu doit faire pour revendiquer cette appartenance comprend par conséquent les attitudes, les valeurs, et les comportements que la société assigne à une personne et à toutes les personnes qui occupent ce statut. Même si un individu agit en fonction de son statut actuel, il possède également des statuts latents qui ne sont pas apparents, mais qui font partie intégrante de l'équipement culturel de l'individu. Ralph Linton, Les fondements culturels de la personnalité, Paris, Dunod, 1977, pp. 70-72.

67 Ibid., pp. 86-87.

68 Serge Moscovici, L'âge des foules, Bruxelles, Complexe, 1981, pp. 339-340.

69 Ibid., p. 346-347. 
des prêtres, enseignants, militaires et policiers, qui sont déterminants. ${ }^{70}$ Et c'est parce que René-Jean Ravault reconnaît la dynamique de ces deux facteurs au sein des relations interpersonnelles qu'il parle plutôt de rapports de «coerséduction ». Ces derniers jouent un rôle dans les stratégies de reproduction de la structure sociale. ${ }^{71}$ Les agents sociaux les exercent de façon à conserver leurs pouvoirs et privilèges d'une génération à l'autre.

Aussi, si la famille se perpétue par des stratégies de fécondité, des stratégies matrimoniales, des stratégies successorales, des stratégies économiques, elle investit surtout dans des stratégies éducatives. Au sein de l'institution scolaire, la standardisation poussée de l'éducation expose les enfants à des rapports de coerséduction similaires. Cela contribue non seulement à l'uniformisation du façonnement de l'habitus des individus et futurs citoyens, mais favorise également la reproduction d'une distribution inégale du capital culturel. Pour Bourdieu, le capital culturel consiste en un ensemble de ressources informationnelles (de data et de personnes), qu'un réseau durables de relations plus ou moins institutionnalisé reconnaît et utilise pour se donner un pouvoir de mobilisation. ${ }^{72}$ En d'autres termes, les enfants de par leur accession à des réseaux de relations, développent des visions du monde distinctes qui n'offrent pas les mêmes privilèges. «Le système scolaire [...] maintient l'ordre préexistant, c'est-à-dire l'écart entre les élèves dotés de quantités inégales de capital culturel. »73 La compétitivité, les examens, en plus d'engendrer des divisions entre les élèves, permettent aussi de justifier les titres et les certificats qui garantissent non seulement des compétences techniques, mais également des compétences sociales. Si l'institution scolaire a instauré une forme de méritocratie qui valorise les aptitudes individuelles plutôt que les privilèges héréditaires, elle nie l'importance de l'héritage culturel dans l'accession au titre scolaire. L'accessibilité généralisée de l'éducation scolaire basée sur l'idéologie du service public et de la méritocratie rend légitime, au nom de l'universel, le pouvoir des classes privilégiées. ${ }^{74}$

Ainsi, les individus au contact des institutions sociales se voient imposés l'apprentissage d'un système de comportements. La culture qu'ils y acquièrent est structurée par un système de valeurs, lui-même actualisé dans des normes. Elle a pour fonction d'édicter un code de conduites sociales qui régissent et facilitent les interactions entre individus. Elle concourt à leur intégration sociale. Ce processus d'enculturation est alimenté par des systèmes idéologiques variés (dont la religion). Il contribue à la stabilité du caractère et du comportement des individus ainsi qu'à la conformité de signification que les membres d'une société accordent à leurs actions,

70 René-Jean Ravault, «Communication dans le monde : un rêve américain... », In Dictionnaire critique de la communication, sous la dir. de Lucien Sfez, Paris, PUF, 1993, p. 78.

71 Pierre Bourdieu, Raisons pratiques : Sur la théorie de l'action, Paris, Seuil, 1994, p. 39.

72 Pierre Bourdieu, Réponses : Pour une anthropologie réflexive, Paris, Seuil, 1992, pp. 94-95.

73 Ibid., p. 40.

74 Ibid., p. 44. 
croyances, et institutions. Il favorise la résolution des problèmes de la vie en groupe. ${ }^{75}$

La subjectivité socialisée ou la grille de décodage acquise par enculturation protège l'identité et tend à fixer ses représentations dans le temps et l'espace. L'attachement identitaire facilite l'appropriation des représentations, c'est-à-dire des conceptions de la « réalité » avec lesquelles des degrés variés de cohérence vont procéder ou non à l'affirmation de sens ${ }^{76}$. En d'autres termes, la représentation constitue une sorte de cliché photographique du présent. Tandis que l'attachement à une appartenance identitaire participe à la reproduction de la signification des choses dans le discours, tout en préservant l'illusion de la conservation. L'identité se préserve ainsi en se reproduisant dans l'identique et en se reconnaissant dans le particulier. En procédant de la sorte, l'identité et la représentation contribue à faire coïncider le moi avec lui-même, l'acteur avec le social, et les mots avec les choses, de façon à conjurer le désordre. La possibilité d'identifier des objets connus ainsi que des séquences de relations conceptuelles crée de l'information parce qu'elle répond aux attentes et aux critères de prévisibilité des acteurs sociaux. ${ }^{77}$ Notre langue, notre culture, notre histoire, etc., constituent autant de filtres apposés sur le fond de notre carte de fond radar qui structurent notre perception. Pour le moment nous dirons donc que l'on ne reconnaît que les connaissances qui font en quelque sorte partie de soi.

\section{2-3.3 Ouverture au changement d'affiliation sur le plan religieux}

\section{$\underline{\text { Retour à la table des matières }}$}

On peut ainsi croire que l'ouverture à ce qui n'est pas soi peut provenir d'une sensibilisation particulière dans le processus de socialisation de l'enfant qui lui permet de concevoir une remise en question de ses croyances. Les études menées par Sultan démontrent que les nouveaux adeptes religieux sont très souvent laïcs et ne possèdent que des connaissances très limitées de la religion. ${ }^{78}$ Ou encore, ils proviennent de mariages mixtes, ou de famille où les deux parents n'adhèrent pas aux mêmes croyances. ${ }^{79}$ Nous présumons que les rapports de coerséduction exercés par les parents et le système d'éducation ont pour but de préserver le sens des représentations et de

75 Ralph Linton, Les fondements culturels de la personnalité, Paris, Dunod, 1977, p. XVIII et XXX.

76 François Laplantine, Je, nous et les autres : Être humain au-delà des appartenances, Paris, Le Pommier-Fayard, 1999, pp. 137-140.

77 Daniel Bougnoux, Introduction aux sciences de la communication, Paris, La découverte, 1998, p. 79-81.

78 Madeleine Sultán, «Choosing Islam : a Study of Sweedish Converts », Social Compass, 46(3), 1999, p. 326.

79 John Leland, « Searching for a Holy Spirit », Newsweek, May 8, 2000, p. 62. 
perpétuer les habitudes, religieuses ou autres, afin de prévenir tout transfert du sens de cette appartenance. C'est ce qui expliquerait pourquoi la majorité des gens a tendance à ne pas se convertir et à conserver la religion des parents. ${ }^{80}$ Bien qu'ils puissent se questionner sur leur foi, il n'y a qu'un nombre restreint de personnes qui changent d'affiliation religieuse. ${ }^{81}$ On peut donc penser que l'acquisition d'un nouvel habitus religieux est en partie attribuable à l'impossibilité, lors de la socialisation primaire, de s'identifier complètement à une pratique bien déterminée. Nous ne disons pas que la conversion religieuse est attribuable à une défaillance de la socialisation primaire. Simplement que l'enfant qui est sollicité par la relativité des points de vue en matière de religion, risque de faire preuve d'une plus grande ouverture d'esprit dans ce domaine. Mais cela ne sera pas suffisant pour qu'il se convertisse. Il faut surtout que cette éventualité devienne significative pour lui dans un contexte donné et en relation aux autres. Tant que le discours à propos de la conversion n'atteint pas le seuil de la sensibilité d'une personne, il ne peut être considéré.

\section{2-3.4 La communication intrapersonnelle}

$\underline{\text { Retour à la table des matières }}$

Comme nous l'avons laissé entendre, les paroles ne font sens que dans la mesure où celui ou celle qui en accuse réception y est sensible. Parfois, cette sensibilité est centrée sur la confirmation de l'identité, l'individu étant gratifié par la reconnaissance des caractéristiques uniques de sa personnalité. Parfois, elle n'est pas vraiment orientée vers un intérêt quelconque. L'individu qui se reconnaît lui-même et qui reconnaît les siens exerce le plus souvent sa sensibilité afin de mettre à jour le cheminement de son être ainsi que sa conception des autres et de l'extériorité. Que l'identité revendique sa singularité, ou démontre un caractère unifié, elle présente, dans tous les cas, un rapport à autrui. ${ }^{82}$

Aussi, selon Marc Augé, la relation entre l'identité et l'altérité correspond à un acte réflexif. Il prend la forme d'un retour sur les questions qui nous préoccupent, et que les autres éclairent sous un nouveau jour, par leurs points de vue différents. En bref, les autres peuvent contribuer à nous faire découvrir des aspects de nous-mêmes inconnus jusqu'alors. Cet apport risque d'être méconnu si l'interaction avec l'autre se construit sur la base du rejet des différences ou de l'oubli de soi. L'échange avec l'autre peut toutefois devenir une source de réflexion qui alimente la communication intrapersonnelle.

80 Stefano Allievi, Les convertis à l'islam, les nouveaux musulmans d'Europe, Paris, l'Harmattan, 1998, p. 35.

81 Bien sûr, on ne parle pas ici de ces conversions massives qui s'effectuent dans des contextes parti-culiers de guerre ou de dissolution sévère de l'environnement social.

82 Marc Augé, Le sens des autres : Actualité de l'anthropologie, Paris, Fayard, 1994, p. 59. 
Lorsque nous parlons de communication, nous négligeons souvent de tenir compte de cette dimension, comme si le sujet était absent de lui-même, alors qu'il essaie constamment d'attribuer des significations à ce qui l'entoure et en relation aux autres. En interagissant constamment avec nous-mêmes, nous arrivons à nous distancier de notre être de façon à repenser nos échanges avec nous-mêmes et les autres, en plus de rechercher ce que les autres pensent de nous. Nous nous investissons à essayer de saisir la spirale des échanges verbaux qui fait dire ceci, plutôt que cela, à une telle personne, à un moment précis. ${ }^{83}$ Par exemple, un homme en introspection peut ainsi prendre conscience que sa femme ne le comprend pas. Cela pourra signifier qu'il pense qu'elle ne réalise pas qu'il se sent négligé, ou encore qu'elle ne sait pas qu'il l'aime, ou encore qu'il croit qu'elle croit qu'il est trop ferme alors qu'il se veut prudent, etc.

Aussi, pour R.D. Laing, la façon dont nous interprétons les différentes interactions verbales auxquelles nous participons, contribue à la création de spirales métacommunicationnelles. L'ensemble des interactions qui incluent la gestuelle, l'intention que l'on prête à l'autre, la nature des énoncés des uns, la médiation des autres, engendre un va-et-vient de codes. Le transfert d'information s'effectue en une superposition de ces codes historiquement déterminés, où interviennent des prises de décisions, ce qui résulte en un surcode dont l'effet est imprévisible. ${ }^{84}$ Le sens de l'échange est affecté non seulement par le surcode qui crée des rapports de signification supplémentaires, mais aussi par la contrainte des structures de la langue et la carte radar individuelle. Pour Lucien Sfez, la libre décision (ou encore l'objet de l'échange) est sujette aux variations découlant du surcode (que l'on désigne également par le terme interprétant). Elle est également exposée à des codes (ou signes), tant au niveau de la forme, par la langue, qu'en ce qui a trait au contenu, par le choix d'éléments découlant de l'adoption de paradigmes

particuliers, de représentations, de schèmes de valeurs. ${ }^{85}$ Aussi suivant leur code, deux acteurs sociaux qui entrent en interrelation en se parlant deviennent des actants

83 R.D. Laing, Interpersonal Perception : A Theory, and a Method of Research, New York, Harper, 1966, p. 30-35.

84 Lucien Sfez, La décision, Paris, PUF, QSJ ?, 1984, pp. 110-115.

85 Nous croyons que cette explication de Sfez sur la reconnaissance des codes est beaucoup plus proche de la sémiologie peircienne que celle conceptualisée par Saussure. Pour ce dernier, le signe, comme par exemple un panneau d'arrêt, constitue une chose sur le plan de l'expression (signifiant), mais signifie autre chose sur le plan du contenu (signifié). Peirce apporte à cette idée des nuances importantes. Il suggère plutôt que le signe ne soit pas attribuable à une volonté particulière, mais peut émaner de l'univers entier (par exemple un beau coucher de soleil peut donner l'idée qu'il fera beau demain). La communication n'est ainsi plus restreinte aux propos explicites que deux personnes s'échangent, mais à tout ce qui est perceptible. L'interprétant n'est pas le récepteur, mais le sur-code qui permet de rapporter tel signe à tel objet. La position élaborée par Peirce élargit la vitalité des signes au-delà de la simple relation émetteur destinataire ; pour lui nous allons de signe en signe et les objets peuvent servir de signes aux autres. Daniel Bougnoux, Introduction aux sciences de la communication, Paris, La découverte, 1998, p. 31-33. 
dont les deux rationalités vont se confronter et où chacun va traduire le message de l'autre en fonction de son système de déchiffrement. C'est cette traduction qui est porteuse d'effets de sens ou d'incommunicabilité. Ainsi, même si les actants partagent des codes ou signes similaires, l'interprétation de leur propos fluctue en fonction de qui ils sont, et selon la relation dialectique qu'ils entretiennent avec eux-mêmes, les autres et leur environnement dans le temps. Pour arriver à se comprendre, ils doivent se livrer à de véritables opérations de transformation de leur dialecte, les uns envers les autres.

\section{2-3.5 La communication interpersonnelle}

\section{$\underline{\text { Retour à la table des matières }}$}

Les généralisations symboliques associées à un signe et qui découlent d'un paradigme, constituent des expressions qui orientent la pensée sans pour autant dicter une interprétation stricte. En d'autres termes, les membres de deux écoles de pensée qui envisagent un problème selon deux perspectives antinomiques (en communication, les partisans qui adhèrent à la théorie des effets des médias versus ceux qui cherchent à expliquer le processus de la réception), vont utiliser les mêmes expressions sans pour autant référer aux mêmes contenus. Cette situation crée également des problèmes de traduction considérables.

Bien que nous n'estimons pas que la signification des idées à l'intérieur d'une même école de pensée soit interprétée de façon monolithique, la base des échanges risque de s'effectuer à partir d'orientations communes. Considérant notre impossibilité, d'accéder à la connaissance objective de la « réalité », nous élaborons des schèmes d'analyse par lesquels nous interprétons ce que nous percevons et auxquels nous adhérons. ${ }^{86}$ C'est en établissant des systèmes de règles et de critères que nous parvenons à créer un certain ordre qui nous permet d'établir des relations de similarité, de dissemblance, ou autres. Lorsqu'il y a présence de perceptions différentes face aux mêmes stimuli, cela ne signifie pas que les perceptions soient aléatoires. Des perceptions ayant subi l'épreuve de l'usage du groupe peuvent être communiquées de génération en génération. L'étendue de la formation ainsi que l'expérience préalable interviennent également au niveau de l'interprétation. Par exemple, la vue de petites gouttes d'eau sur une échelle numérique peut paraître banale pour toute personne ne connaissant pas les chambres de Wilson. ${ }^{87}$ La position de la personne ayant acquis la connaissance de cet instrument sera différente parce qu'elle interprétera ces gouttelet-

\footnotetext{
86 Thomas S. Kuhn, La structure des révolutions scientifiques, Paris, Flammarion, 1983, pp. $265-$ 275 .

$87 \quad$ Ibid., p. 268.
} 
tes d'eau, comme des manifestations de la présence d'électrons et autres particules. ${ }^{88}$ Ainsi, des individus vivant dans des communautés d'interprétations différentes, qui traduisent selon leur identification des points de vue incommensurables, auront de la difficulté à prouver la supériorité de leurs arguments par la discussion. Chaque partie devra donc user de beaucoup de persuasion pour convertir l'autre parce que leurs oppositions témoignent des différences d'interprétations et de l'application divergente de règles admises. Bien qu'un débat puisse porter sur un objet particulier, il soulève en fait des désaccords à propos des prémisses, c'est-à-dire sur l'interaction entre les expériences particulières communes au groupe et un ensemble particulier de valeurs communes. Lorsque des parties divergentes découvrent que leurs interprétations ou que l'application des règles admises ne sont pas les mêmes, ils restent confrontés aux problèmes de traduction. S'ils arrivent à isoler les secteurs qui les opposent dans leurs communications en stimulant, par empathie, de nouveaux angles d'approche pour appréhender leurs différents, il est possible que la prise de conscience de la position de l'autre devienne un outil de persuasion, voire de conversion. Il est toutefois rare que des tenants de visions opposées manifeste dans leurs échanges autant d'empathie, c'est-à-dire qu'ils en viennent à ressentir ce que les autres conçoivent selon les mêmes présupposés. La difficulté des mots à rendre compte de l'expérience des autres complexifie le processus d'identification aux points de vue des autres. Par conséquent, les parties opposées font rarement preuve d'autant de diligence. L'identification au groupe a tendance à vouloir tout ramener à soi-même sans égard à l'autre.

\section{2-3.6 Cognition et variations de l'interprétation}

$\underline{\text { Retour à la table des matières }}$

En relation avec ce que nous avons dit précédemment, la communication avec les autres est étroitement liée à la communication que chacun entretient avec lui-même. Sur le plan individuel, la communication intrapersonnelle soit la faculté d'acquérir et de traduire des «données » pour les rendre compréhensibles, permet aussi de prendre connaissance de l'extériorité, selon la sensibilité des individus. ${ }^{89}$ Ceci a pour fonction de maintenir l'adaptation de ces derniers à leur environnement. Le niveau de complexité d'un tel processus dépend des relations que l'esprit effectue.

88 Cet exemple illustre bien, selon nous, la loi d'Ashby selon laquelle le niveau de complexité de la relation qu'entretient un individu avec son environnement dépend du niveau de complexité propre à son système d'interprétation, de «compréhension » du monde et de lui-même. Cette explication est inspirée des propos de Lee Thayer, On Communication : Essays in Understanding, Norwood, (New Jersey), Ablex, 1987, p. 75. 
Tant que l'esprit n'est pas confronté à un événement ou à un ensemble de « data » pour lequel il n'a aucun repère, il a tendance à répéter ses vieilles formules. Un peu comme le vieux cheval qui reprend toujours le même chemin pour retourner à l'écurie, l'esprit effectue un tri entre les catégories qui lui apparaissent pertinentes ou pas, afin d'établir des connections et tend, si on le laisse à lui-même, à reproduire les mêmes patrons de cognition. Il en coûte donc beaucoup moins de revenir à ses habitudes plutôt que de changer délibérément sa façon de penser.

Aussi pour que l'esprit consente à s'aventurer en terrain inconnu, il va au départ prendre conscience d'anomalies qu'il ne peut résoudre à l'intérieur du cadre conceptuel présent dans son esprit. C'est ce qu'avance Thomas S. Kuhn dans son livre La structure des révolutions scientifiques où il démontre comment les scientifiques en arrivent à se convertir à un autre paradigme dans le cours de leur recherche. Nous allons ici adapter ces propos au processus de conversion religieuse.

Tout d'abord Kuhn spécifie comme nous l'avons dit précédemment que la perception doit être affinée pour remarquer de nouveaux phénomènes. ${ }^{90}$ Un dentiste, par exemple, remarquera rapidement les problèmes de dentition des personnes alors que le dermatologue notera facilement leurs infections cutanées. Toutefois lorsqu'un nouveau phénomène surgit, comme cela a été le cas pour le SIDA et maintenant pour le Syndrome Respiratoire Aigu Sévère (SRAS), les médecins ont beaucoup de difficultés à établir au départ de quoi il s'agit, ne serait-ce que les symptômes. Il est donc plus facile de prendre connaissance d'un problème classique, que de disposer d'une disponibilité et d'une ouverture sur le plan conceptuel pour résoudre une énigme. Éventuellement, l'observation et la conceptualisation vont conduire à l'élaboration d'une nouvelle théorie et peut-être même à une nouvelle façon de concevoir le problème. Mais cela prend généralement du temps. Les catégories conceptuelles doivent s'ajuster pour expliquer les effets de ce qui apparaît désormais anormal, avant de vraiment envisager l'existence d'un nouveau phénomène.

Pour parvenir à cela, l'esprit doit faire preuve d'ouverture ou encore d'hospitalité. Pour Theodore Zeldin, il ne s'agit pas d'un simple divertissement, mais d'une disposition de l'esprit à accueillir des idées, des opinions, des traditions inconnues qui vont interférer avec la vision du monde initiale des personnes et ce qu'ils sont. ${ }^{91}$ Cette hospitalité profonde, bien plus qu'une forme de politesse, n'est possible que si l'esprit travaille de façon inhabituelle. Il doit faire preuve de vigilance s'il ne veut pas reprendre ses modèles de cognition habituels et agir conformément à la façon dont il perçoit, se souvient, imagine et juge normalement.

Il est difficile de disséquer la pensée, mais, intuitivement, il est possible de saisir certains éléments du processus. On peut ainsi admettre que la perception puisse susciter des questions qui pourront stimuler l'atteinte de nouveaux buts. Mais, le plus sou- 
vent, l'être humain ne s'attarde pas à considérer ces possibilités et classe l'ensemble des «données » qu'il perçoit selon les catégories mentales qu'il reconnaît bien. En procédant de la sorte, les surcharges d'information sont évitées. Même si nous avons l'impression qu'en regardant attentivement il est possible de saisir l'ensemble des éléments constituant l'extériorité en un flash, l'acte de perception se limite à ne considérer que les stimuli conformes aux visions du monde. En d'autres termes, les paradigmes servent de repères à l'interprétation, sensiblement de la même façon que les cartes de navigation servent à orienter. Les sens prennent connaissance de l'extériorité comme les ondes d'un radar qui, une fois réfléchit, retourne vers le récepteur. Les signaux sont ainsi interprétés conformément à la «carte-radar» individuelle. Cette carte qui comporte les généralisations symboliques qui découlent d'un paradigme, c'est-à-dire d'un ensemble de valeurs, de croyances communes auxquelles adhèrent les membres d'un groupe orientent non seulement le sens de l'interprétation, mais crée aussi un cadre aux possibilités de l'acte perceptif.

Lorsque la personne qui perçoit, entrevoit soudainement des événements pour lesquels elle n'a aucune explication, une anomalie apparaît sur le fond de l'écran radar. Son interprétation du monde est aux prises avec un manque de repères. Si cette incompréhension devient une source de questionnements constants, l'acteur social pourra rechercher la solution à son problème par l'adoption d'un nouveau paradigme interprétatif. Dans bien des cas, cette démarche va entraîner une prise en compte de nouveaux termes, une nouvelle forme de langage expliquant le nouveau cadre explicatif des expériences.

Cet aspect qui a d'ailleurs été relevé par plusieurs chercheurs intéressés par le phénomène de conversion religieuse, a été plus spécialement souligné par André Billette dans son livre Récits et réalités d'une conversion. Après avoir interrogé des convertis presque deux ans après les premiers entretiens, Billette a remarqué chez eux, non seulement l'adoption d'un nouveau schème d'appréhension, mais des traits communs à travers les différents récits de conversion. ${ }^{92}$ Tout d'abord le je parle toujours du moi. Ensuite le je dit avoir changé de je, d'avoir remédié à un mal existentiel (indécision, solitude, conflit), en ayant trouvé un sens à sa vie ou une reconnaissance sociale. Ce récit événementiel particulier n'est pas isolé, mais est en continuité avec une série d'événements fondateurs. Même s'il est présenté comme un signe divin, le converti en est toujours l'initiateur. Dans l'ensemble des récits, on distingue les périodes qui précèdent et qui suivent la conversion. ${ }^{93}$ De plus, le récit est réajusté dans le temps à mesure que de nouvelles expériences sont assimilées en fonction de l'apprentissage du nouveau schème de pensée et de son intégration dans la vie quotidienne. On peut donc en déduire que l'esprit risque d'être d'autant plus fébrile face aux variations de mémoire, de perception, d'imagination, d'intuition et de jugement

92 André Billette, Récits et réalités d'une conversion, Montréal, Presses de l'Université de Montréal, 1975, p. 13.

93 André Billette, « Se raconter une histoire... : Pour une analyse révisée de la conversion », Social Compass, XX111, 1976/1, pp. 50-51. 
que la vision du monde nouvellement adoptée constitue un modèle cognitif inhabituel.

\section{2-3.6.1 La mémoire}

Retour à la table des matières

Pendant la période d'assimilation du nouveau paradigme, on peut supposer que la mémoire est sensible à des états de tension attribuables à l'incertitude. Ordinairement utilisée pour retracer les références dont une personne a besoin pour donner une signification à son existence, la mémoire peut se retrouver sans repères. Par exemple, je me souviens lors d'un échange culturel au Mexique d'avoir senti un tremblement de terre pendant une projection cinématographique. Les gens dans la salle s'étaient mis à hurler et à courir vers la sortie. Ne sachant que faire, n'ayant jamais vécu ce type d'expérience, j'avais conclu que je devais gérer cet état de stress en adoptant le même comportement que les membres de ma famille d'accueil, puisque ces gens m'apparaissaient fort sensés. Ils n'ont pas bougé et sont restés assis bien calmes tout comme moi. Ils m'ont expliqué par la suite que les situations de panique sont également responsables de bien des cas de mortalité.

On peut déduire de cette expérience inusitée, que ma mémoire, dans l'impossibilité de résoudre ce problème s'est référée à un mode de cognition déjà connu, le mimétisme. J'ai ainsi profité de la mémoire des autres. Dans d'autres situations, la mémoire aurait pu tout aussi bien emprunter d'autres voies ; celles de la manipulation, de la sous-utilisation ou encore celle de l'auto-référence continuelle. ${ }^{94}$ Mais si elle peut trafiquer les souvenirs, les oublier ou respecter ses références passées, il est possible aussi qu'elle s'ouvre à de nouvelles façons de faire. La mémoire n'est pas qu'orientée vers le passé : si elle en tire sa source, elle peut par la suite être adaptée à de nouveaux contextes.

Giddens pense que cette possibilité s'est accrue avec l'avènement de l'écriture. Selon lui, dans la culture orale, la tradition est soumise aux contraintes inhérentes à la mémoire. ${ }^{95}$ On procède par la répétition d'un discours qui revient continuellement sur lui-même au passage des saisons et du temps. Ce discours est constitutif de l'identité parce qu'il contribue à susciter chez les individus des relations d'appartenance au groupe. L'essor de l'écriture a créé un espace de distanciation entre l'individu et le groupe. Sans cet écart, la réflexion, sine qua non de la conversion, semble pratiquement impossible. 
Mais avec l'invention de l'écriture, le présent n'est plus lié au quotidien. La possibilité d'une distanciation spatio-temporelle s'est instaurée, rendant ainsi possible l'appropriation réflexive du savoir. ${ }^{96}$ Le discours pouvait dès lors être revisité plus librement. Mais cette pratique allait s'inscrire à l'encontre de la fixité de l'ordre social pré-moderne qui impose la clarification de la tradition, plutôt que sa remise en question. La possibilité d'exercer l'acte réflexif avec plus de latitude par rapport à une vision traditionnelle du monde, crée une distanciation entre soi et la communauté d'appartenance, qui permet d'entrevoir des variations multiples de l'être.

\section{2-3.6.2 L'imagination}

$\underline{\text { Retour à la table des matières }}$

L'importance accordée à la mémoire se relativise. On la laisse s'estomper pour faire place à plus d'imagination. L'esprit, moins contraint par les règles passées, moins inhibé, envisage plus facilement d'innover en s'orientant vers le futur. Il peut alors se découvrir sous un nouveau jour, ou encore se perdre dans un monde parallèle incompréhensible pour les autres. C'est pourquoi l'imagination, qui fait référence à l'inconnu, suscite souvent de la crainte. Par exemple, dans la Bible (Genèse 6:5), l'imagination est condamnée parce qu'elle est considérée comme une source de désobéissance. ${ }^{97}$ Elle est également perçue comme opposée à la raison. John Locke, par exemple, pourtant ennemi de tous les dogmes, ne pouvait concevoir que l'on puisse imaginer un monde sans Dieu. ${ }^{98}$ Les Romantiques 99 quant à eux y transposaient de grands espoirs. Ils pensaient qu'elle pouvait servir à percer les mystères de l'univers. 100 Or, si, elle peut confondre le conditionnement de l'esprit, l'imagination, si elle n'a pas de cadre interprétatif, peut dépasser les normes socialement acceptées. La conversion, en tant que pratique imaginative qui ne s'inscrit pas dans le sens de la conformité, peut conduire à l'exclusion sociale. Mais elle peut aussi constituer une issue constructive pour le converti si elle lui offre un nouveau cadre d'appréhension de la « réalité » qui lui permette d'interpréter ses incompréhensions.

\footnotetext{
96 Ibid., p. 44.

97 Theodore Zeldin, An Intimate History of Humanity, New York, Harper Collins, 1994, p. 441.

98 Ibid., p. 441.

99 Nous faisons référence au courant littéraire du moyen âge, qui regroupe des écrivains qui s'inspirent de la chevalerie et du christianisme, et qui s'opposent aux classiques. Petit Robert 1, dictionnaire de la langue française.

100 Theodore Zeldin, An Intimate History of Humanity, New York, Harper Collins, 1994, p. 441.
} 


\section{2-3.6.3 Perception renouvelée et intuition}

\section{Retour à la table des matières}

Le plus souvent, la solution recherchée parce qu'elle ne peut pas faire complètement abstraction des attachements identitaires risque d'en faire partie. La démarche sera toutefois inusitée dans la vie du converti. En premier lieu, c'est sa perception des choses qui sera affectée. Des phénomènes incompréhensibles vont surgir qui ne pourront être interprétés comme à l'accoutumée. L'incertitude engendrée par cette situation peut stimuler l'esprit à s'ouvrir intuitivement à autre chose. Toutes les fonctions cognitives de l'esprit seront alors mises à contribution, y compris la mémoire. Puisque l'impossibilité d'attribuer un sens est inconfortable, l'esprit, plutôt que de veiller au maintien perpétuel des particularités de l'identité primaire, va être mieux disposé à confronter sa vision des choses avec celle des autres.

Un médecin catholique québécois, par exemple, œuvrant à l'intérieur d'un réseau de relations bien définies pourrait en venir, dans le cadre de son travail, à développer un réseau de relations secondaires au cours d'un séjour en pays musulman. Ce réseau secondaire de relations pourrait créer des conditions propices à la ré-interprétation de la biographie personnelle.

Mais, contrairement au dialogue quotidien qui se situe, la plupart du temps, dans le respect des conventions sociales acceptées, la remise en question de soi exige beaucoup plus. La concession identitaire signifie non seulement de se risquer à exposer le conglomérat d'idées reconnues comme nôtre, mais aussi d'être séduit ou contraint par l'efficacité de la raison des autres. Ainsi, pour connaître, l'esprit doit se laisser convertir dans la chose connue, se mettre à la place d'autrui, s'exposer à la force d'autres raisons. La persuasion réside dans cette capacité communicative à faire coïncider ses idées propres et les valeurs qu'elles sous-tendent, avec un aspect des valeurs des autres. ${ }^{101}$ Celui qui abandonne ses anciennes idées pour se laisser séduire par les porteurs de nouvelles représentations, réactualise l'expérience délaissée, dans un monde de discours nouvellement partagés. Cette ouverture d'esprit qui fait correspondre l'autre à soi sur le plan éthique, révèle l'universalité des représentations. Elle constitue ainsi un facteur contribuant à l'unification des uns aux autres, nonobstant leurs apparences ou leur provenance. De plus, elle sensibilise à l'exploration d'autres possibilités en marge de l'identité, appréhendées, jusqu'alors, avec peu de considération.

101 Humberto Giannini, La tolérance : Pour un humanisme hérétique, sous la dir. de Claude Sahel, Paris, Éditions Autrement, 1996, p. 21. 


\section{2-3.6.4 Le jugement}

\section{Retour à la table des matières}

La construction des « réalités personnelles » n'est donc pas uniquement déterminée par l'intériorisation dans la conscience des conditionnements passés. Elle s'élabore aussi en fonction de la possibilité ou de l'impossibilité à interpréter sa vision du monde conformément à celle des membres des groupes de relations auxquels nous appartenons ou adhérons par choix. Ce que nous transigeons en vue de l'édification de nos projets avec les autres, est évidemment sujet aux fluctuations imposées par des événements circonstanciels ainsi que l'aptitude avec laquelle les individus entrent en communication avec eux-mêmes et les autres. La réflexivité et l'échange incluent une disposition de l'esprit à se rendre disponible pour écouter et se laisser convaincre par la rationalité des propos des autres.

Contrairement à l'animal, l'humain use de sa conscience pour s'orienter vers de nouveaux systèmes de connaissances. Cette dernière, en interaction constante auprès du jugement oriente la prospection intellectuelle, la sensibilité ainsi que l'adhésion. 102 Pour Georges Bastide, la relation entre la foi de conscience et le jugement sous-tend l'activité judicatoire qui se présente comme suit : premièrement, la conscience croit que ... c'est-à-dire que, de par les intérêts de la conscience qui juge et établit des liens, le donné de la masse sensible est non perçu ou se constitue en une représentation structurée ; deuxièmement, la conscience croit à ... c'est-à-dire qu'elle assigne une valeur à la représentation selon ce qui est affirmé ; troisièmement, la conscience croit en ... relève de l'acte de confiance en soi ou en l'autre et son témoignage. Croire que ... correspond à l'aspect gnoséologique de cette foi immanente, c'est-à-dire à la coloration affective qui attire par «séduction » ou circonscrit par coercition le champ d'exploration. Croire à ... quelque chose, au bien, au mal, représente l'aspect axiologique de la conscience inclusive de la dimension éthique. Croire en ... soi, en l'autre ou en Dieu, sert de motivation à l'action et réfère à l'aspect ontologique de la conscience c'est-à-dire à l'être indépendamment de ses déterminations. Les trois aspects que nous venons de définir, soit la prédisposition à percevoir les objets de préoccupation, la possibilité de les juger sur le plan éthique, et la disposition à concevoir ces objets sous un angle nouveau, se retrouvent concurremment dans l'acte de conscience et dans la production de sens.

En d'autres termes, l'humain crée des rapports au sens en décodant sa "réalité » selon différentes opérations cognitives. Afin d'éviter de perdre le fil de la conversation qu'il entretient avec lui-même, il oriente sa perception, sa mémoire, son imagina-

102 Georges Bastide, La conversion spirituelle, Presses universitaires de France, Paris, 1966, pp. 37. 
tion ainsi que son jugement de façon à confirmer sa vision du monde. Mais lorsqu'il fait preuve d'hospitalité envers l'autre malgré ses différences, il peut également reconnaître l'expression des valeurs de ce dernier comme sienne. Toutefois, quand l'ensemble des représentations n'arrivent pas à interpréter des situations inhabituelles, comme celles associées à la mort, à la souffrance, la personne peut se retrouver en état de conflit d'interprétation. De même, lorsque l'interprétation des valeurs qui l'unit à des groupes, devient une source de conflits entre les membres, selon l'évolution du contexte. Cette crise interprétative peut prédisposer à s'ouvrir à ces autres et à se laisser «séduire » par eux, surtout s'ils nous sont apparus à un moment donné comme nôtres, tant la similarité de leurs représentations et de leur interprétation éthique semblaient proches.

\section{2-4 Appréhension du contexte social}

Retour à la table des matières

Si le sens des représentations individuelles est affecté par les relations que nous entretenons par coerséduction les uns envers les autres, il varie également selon le contexte. Dans les sociétés occidentales, la remise en question de la transcendance signifie aussi que le sens des valeurs ainsi que des projets qui unissent l'ensemble des membres d'une communauté ne fait plus l'unanimité. Les acteurs sociaux se retrouvent bien souvent laissés à eux-mêmes pour donner une signification à leur existence. Certains en arrivent même à se sentir complètement isolés des autres. Par ailleurs, si certains d'entre eux s'investissent sans cesse dans la maximisation de leurs intérêts individuels ou collectifs, la portée de leurs actions est désormais limitée à un environnement qu'ils ne connaissent ou ne contrôlent que partiellement. Ainsi, la participation financière des syndicats, dans divers fonds de placement, ces dernières années, a affaibli leur pouvoir de négociation. Cette situation a compromis la crédibilité du mouvement ouvrier, dans l'arène des débats sociaux et politiques. La nature de tels changements dans le contexte actuel de la modernité portent ainsi atteinte au principe d'unité de vie sociale ainsi qu'aux projets collectifs. C'est pourquoi la notion de l'acteur social porteur d'une rationalité, fait plutôt place maintenant à celle où chacun d'entre eux défend des intérêts diversifiés et obéit à des identifications de groupe. ${ }^{103}$ Cela signifie qu'ils s'adaptent à la nouveauté en lui donnant un sens conforme à celui du groupe auquel ils s'identifient et qui correspond, dans bien des cas, à la vision du monde acquise par coerséduction au sein des milieux d'appartenance. Néanmoins, des situations de conflits peuvent surgir en relation aux autres selon l'évaluation des transformations contextuelles. Ce changement peut motiver l'acteur social à rechercher une nouvelle façon d'interpréter et, conséquemment, de s'interpréter.

103 Alain Touraine, Le retour de l'acteur : Essai de sociologie, Paris, Fayard, 1984, p. 93. 


\section{2-4.1 Contexte social et visions du monde}

$\underline{\text { Retour à la table des matières }}$

L'esprit entretient une relation dialectique avec son environnement. Si nous avons parlé plus spécifiquement de la relation que les individus entretiennent avec des groupes, il faut savoir que, tous, partagent aussi, à l'intérieur des sociétés, un ensemble de codes sociaux. Et c'est à partir de cette Weltanschauung imposée par coerséduction par les autorités politiques, administratives, religieuses ou autres qu'ils construisent leur « réalité ». Ces univers de significations que l'on nomme souvent «culture » se remarquent dans le mode de fonctionnement des institutions et dans les valeurs que ces organisations défendent. Cette activité, qui est également le fruit d'une quantité d'opérations cognitives, peut s'effectuer selon des registres d'interprétation qui veilleront au respect de la tradition ou encore feront place à l'innovation et au consensus.

Pour expliquer comment cela se produit, nous allons examiner avec quelle latitude contextuelle les acteurs sociaux appartenant à une société plutôt qu'à une autre intériorisent leur propension à se distancier de leur Weltanschauung ; c'est-à-dire à réfléchir sur cette conscience collective intériorisée qui pense à leur place et qui les empêchent de se convertir. ${ }^{104}$ Plus concrètement, cela se traduit sur le plan religieux, par la possibilité d'exercer ou non une certaine liberté de conscience. ${ }^{105}$ Les adeptes de la vision traditionnelle de la religion, font valoir que la liberté illimitée de conscience compromet la notion de révélation qui justifie l'autorité transcendante de la parole de Dieu. Tandis que, les personnes qui adhèrent à une vision plus moderne de cette problématique, conçoivent que l'homme, à l'image de Dieu, peut exercer avec sincérité son jugement moral en accord avec sa conscience individuelle.

Ce débat à propos de la liberté de conscience abordée d'un point de vue transcendant, puis dans une perspective immanente et à sur lequel nous devrons revenir, implique deux orientations cognitives auxquelles correspondent deux types d'organisations sociales. Tönnies utilise le terme Gemeinschaft pour décrire les spécificités des univers culturels des sociétés traditionnelles et Gesellschaft les caractéristiques des sociétés modernes.

104 René-Jean Ravault, «Développement durable, communication et réception active», In Communication et développement international, sous la dir. de Thérèse Paquet-Sévigny, Sainte-Foy, (Qué.), Presses de l’Université du Québec, 1996, p. 64.

105 Notons toutefois que, pour l'Église, la subjectivité de la conscience humaine ne peut établir la source du bien et du mal. Aussi, selon cette institution, la vérité objective est attribuable à la volonté divine puisqu'elle ne peut émaner de l'humain. Luc Ferry, L'Homme-Dieu ou le Sens de la vie, Paris, Grasset, 1996, pp. 52-54. 
Dans les premières, le fondement de l'ordre moral est essentiellement révélé. Le pouvoir religieux se retrouve en position de dicter l'échelle de la hiérarchie sociale, de haut en bas. À l'intérieur de ces sociétés, l'Église jouit généralement d'une grande autorité et les intérêts de cet institution sont le plus souvent défendus par la royauté. La transcendance divine incarnée par la royauté distribue les rôles sociaux et garantit la justesse de l'ordre humain. Puisque les rôles sont ainsi fixés d'avance, ils ne peuvent être contestés; ce qui oblige donc un comportement social bien précis. ${ }^{106} \mathrm{La}$ communauté impose de façon plus répressive le sens des conventions sociales et les limites de l'action personnelle de ses membres. L'attitude psychologique qu'il convient d'adopter ne cherche pas à comprendre mais à se conformer. 107 " La société traditionnelle est certes très contraignante, mais elle est aussi très sécurisante car elle fournit constamment des repères de bonne conduite personnelle et sociale. » ${ }^{108}$

Lorsque, par un concours de circonstances, les membres d'une société en viennent à rejeter l'idée de l'autorité divine ainsi que les formes d'institutions «transcendantalisées » s'y rattachant, ils remettent en cause le principe même qui maintient la cohésion sociale et les inégalités. Il suffit de penser aux bouleversements sociaux advenus à la suite des Révolution américaine, française et russe. Cette nouvelle façon de concevoir le monde marque le passage des sociétés traditionnelles vers la modernité. Elle ouvre la voie vers la reconnaissance de principes immanents, humains et constants, qui stimule l'essor de systèmes politiques démocratiques. Désormais, « [...] l'ordre social et humain n'est plus établi en fonction de la transcendance divine mais par l'action humaine. »109 Dans le monde moderne, la religion ne devient qu'une sphère d'activités parmi d'autres pouvant organiser l'ordre social. Le pouvoir des individus est attribué en fonction de leurs compétences, même si celles-ci peuvent se rattacher à un ordre transcendant.

En Occident, le passage des sociétés traditionnelles vers la modernité va se faire sur plusieurs siècles, selon l'évolution des acteurs sociaux dans les différents secteurs de l'activité sociale. Les sociétés modernes vont se développer à mesure que les conditions sociales et économiques qui lui sont propres émergent.

Ainsi libérés de l'emprise de la tradition imposant la «fétichisation » d'un Dieu, les hommes et les femmes vont se reconnaître «égaux ». Un nouveau contrat social s'impose. Cette situation entraînera la création de nouvelles institutions ainsi que l'adoption de procédures qui vont permettre à l'ensemble de la société de participer au débat social. La recherche de consensus va stimuler l'édification des fondements de la

106 Paul Attalah, Théories de la communication, histoire, contexte, pouvoir, Sillery, Québec, Téléuniversité, Presses de l'université du Québec, 1989, p. 25.

107 Ibid., p. 25.

108 Ibid., p. 16.

109 Ibid., p. 25. 
nouvelle société démocratique. C'est cette notion de contrat qui caractérise les sociétés de type Gesellschaft.

Les individus des sociétés modernes n'étant plus contraints par le poids de leurs obligations religieuses, ont besoin de garanties quant aux bonnes intentions des autres. Cette considération s'exprime par des ententes qui prennent la forme de contrats. Le non-respect de ces accords se heurte à des sanctions prévues à cet effet. ${ }^{110}$ L'individu des sociétés modernes en ayant à assumer lui-même sa liberté de conscience agit, désormais, en accordant beaucoup plus d'importance à ses intérêts personnels. En conséquence, il s'identifie beaucoup plus difficilement à l'intérêt commun. Ainsi avec l'avènement de la modernité, l'unité sociale n'est plus définie hiérarchiquement en fonction des liens du sang mais négociée selon les termes d'un contrat où l'égalité des individus est reconnue. Il se crée de nouvelles pratiques sociales qui ne peuvent plus se justifier par la tradition.

\section{2-4.2 Pratique renouvelée de l'acte réflexif}

$\underline{\text { Retour à la table des matières }}$

Dans ce nouveau contexte, la réflexivité c'est-à-dire la propension à réfléchir sur soi-même va également s'exercer différemment. Plutôt que de rendre conforme le changement en fonction de la tradition, elle va prendre la forme d'une révision constante de la pensée sur l'action, à la lumière des nouvelles informations. ${ }^{111}$ Du point de vue communicationnel, le va-et-vient entre l'univers de la vie sociale et la réflexion va inciter à une redéfinition de l'action des individus et des groupes.

Ce mode d'adaptation à la nouveauté a une incidence organisationnelle importante qui s'apparente à une réorganisation constante des relations sociales. Elle s'élabore, parce que la dimension du temps ne coïncide plus toujours avec celle du lieu. ${ }^{112}$ Par exemple, j'arrive en Irak et je téléphone à Montréal, malgré le décalage horaire, pour raconter à des auditeurs le début de la guerre de 2003. La mobilité dans l'espace peut accélérer la réflexion sur soi en rapport aux autres.

La modulation de soi dans le temps et l'espace peut engendrer un état qui questionne l'affirmation de l'identité. La foi dans la représentation de sa « réalité » peut être remise en question. L'investissement de soi dans l'appropriation de ce savoir réflexif peut produire des changements de perspective dans l'appréhension de la relation de soi au monde qui conduise aux changements d'orientation des valeurs. Les consé-

\footnotetext{
110 Ibid., p. 15.

111 Anthony Giddens, Les conséquences de la modernité, Paris, l'Harmattan, 1994, p. 45.

112 Ibid., p. 28.
} 
quences d'une telle activité ne sont évidemment pas entièrement connues car la connaissance du déroulement de la vie sociale est partielle. L'exercice d'une réflexion sur des changements qui surviennent rapidement engendre un flot de connaissances instables, voire mutables, qui modifie le sujet. Cette pratique facilite la génération continue d'auto-connaissance. ${ }^{113}$

\section{2-4.2.1 En guise d'exemple...}

\section{$\underline{\text { Retour à la table des matières }}$}

Afin de faciliter la compréhension de ce que nous venons de mentionner, rappelons comment la distanciation que procure les dimensions du temps et de l'espace ajoute une plus-value de sens, un « surcode », à la pratique réflexive. L'exercice que nous proposons consiste en notre réflexion sur la façon dont nous pensons que s'est construit l'intérêt pour l'objet de ce mémoire. Nous verrons ainsi plus concrètement comment la communication intrapersonnelle contribue à la création de nos réalités personnelles et interpersonnelles.

Tout d'abord, pour que l'esprit s'investisse dans la recherche du sens d'un objet qui lui apparaît inconnu jusqu'alors, il doit y être suffisamment intéressé pour faire abstraction de ces autres préoccupations. S'il ne sait comment aborder cet objet de nouveauté, il risque de faire appel en premier lieu à la mémoire afin de rechercher une piste d'interprétation. Comme il serait pratiquement impossible de traiter tous les événements passés d'une vie, l'esprit ne traite que les traces «mnésiques » qu'il a conservées. ${ }^{114}$ Ce faisant, il effectue une sélection en regard du passé qui ravive certaines expériences dans le temps, ou selon des repères dans l'espace. Cette opération qui peut se limiter à l'introspection peut aussi dépasser ce cadre pour devenir une réflexion sur l'appréhension du monde. L'esprit de l'humain peut non seulement se distancier de lui-même, mais aussi s'investir dans un questionnement sur sa façon de concevoir le monde. Cette prise de conscience peut avoir pour effet de susciter de nouvelles perceptions. La pratique réflexive en faisant du neuf avec du vieux, démontre cette capacité chez l'humain de surmonter ses déterminismes afin d'exercer sa liberté. 115

Cette distanciation de soi par la réflexion permet de prendre conscience, non seulement des transformations de l'identité au travers du temps mais aussi des différences de conditionnement identitaire que l'on retrouve dans une culture donnée par rapport à une autre. Cette pratique a d'ailleurs contribué à la réflexion qui sous-tend le pré-

\footnotetext{
113 Ibid., pp. 50-51.

114 Tzvetan Todorov, Mémoire du mal, tentation du bien : Enquête sur le siècle, Paris, Laffont, 2000, p. 133

115 Ibid., p. 136.
} 
sent travail. L'intérêt que nous portons au processus par lequel les acteurs sociaux en viennent à adopter un nouveau paradigme religieux pour guider leur interprétation du monde, semble découlé d'un cumul d'expériences personnelles. J'aimerais vous faire part de trois d'entre elles qui me semblent prépondérantes par rapport à l'objet de ce travail. Elles ont non seulement contribué à modifier ma perception du monde, mais aussi son interprétation. Leur particularité réside dans le fait d'avoir redéfini au fil du temps mon identité tout en participant à me déconditionner, chaque fois, un peu plus en profondeur. Cette redéfinition de l'identité dans l'exercice de la réflexivité s'apparente en partie à ce qui se produit dans le cours d'un processus de conversion religieuse.

Tout d'abord, j'ai étudié sept ans chez les sœurs franciscaines. Cette formation m'a permis, en plus d'acquérir les préceptes de la religion catholique, de vivre selon les règles d'une petite communauté, dont l'œuvre était axée sur l'éducation et la prière. Cela se passait dans le début des années 1970, période à laquelle un vent de réformes a soufflé sur le Québec, balayant hors de l'État le pouvoir clérical. ${ }^{116}$ Le mouvement de laïcisation enclenché a eu l'effet d'une bombe et l'onde de chocs s'est répercutée jusqu'à l'intérieur des cellules familiales. À la même époque, ma famille a cessé d'aller à l'église le dimanche. Les mentalités ont changé, certes, mais le contexte de la Révolution tranquille y a contribué pour beaucoup. J'ai été également emportée dans cette vague de changements. Néanmoins, certaines habitudes sont restées ; prendre de la distance sur ses actes par la réflexion, accorder plus d'importance à ce que l'on est plutôt qu'à ce que l'on a, assumer tant bien que mal ses responsabilités envers ses proches, son environnement, mais aussi ces autres, éloignés, qu'on qualifie souvent à tort, d'étrangers.

Ensuite, ce sont mes voyages qui m’apparaissent avoir été les apprentissages les plus significatifs. Plus spécifiquement, j'ai effectué plusieurs échanges culturels, en Indonésie, en France. Mais c'est surtout celui au Mexique qui, m'a le plus marquée. Même si c'était le premier et que j'étais encore toute jeune (à peine dix-sept ans), c'est la qualité de l'échange avec la famille mexicaine qui m'a accueillie, que je retiens. D'une certaine façon, je m'entendais mieux avec eux qu'avec ma propre famille. Nous cherchions, ensembles, à développer nos intérêts communs. Les différences de socialisation au niveau culturel entre eux et moi, érigées comme des barrières insurmontables au début de notre relation, se sont vite estompées.

L'apprentissage de l'espagnol, l'acceptation d'une autre façon d'appréhender la vie, d'un rapport au monde différent, m'ont révélé l'autre avec ses particularités, tout en me faisant prendre conscience des miennes. Cette constatation m'a permis également de réaliser, que malgré toutes les subtilités culturelles qui nous apparaissent parfois complètement inintelligibles, le plaisir, l'amour, la tendresse, l'amitié, la co-

116 Dans son film, Les invasions barbares, Denis Arcand identifie l'année 1966 comme étant celle où l'on aurait noté une baisse significative du taux de participation à la messe dominicale. Toutefois, dans ma famille mes parents nous ont offert le libre choix sur ce sujet qu'aux alentours de l'année 1972. 
lère, la haine sont exprimés partout. J'ai compris que les êtres vivants, bien qu'ils possèdent des comportements déterminés par la nature de leur environnement culturel, économique ou social, possèdent aussi la capacité de s'en distancier, d'exercer une réflexion par rapport à ces derniers, de leur donner d'autres significations. En outre, ce processus ne peut survenir que par le biais de la communication avec l'autre. L'autre, surtout s'il est très différent, risque de nous introduire dans des sphères de réflexions inattendues qui peuvent nous permettre d'interpréter et agir différemment. Qui plus est, l'intensité avec laquelle une personne s'investit dans cet exercice risque d'être comparable au bouleversement identitaire qu'il provoque. S'investir auprès des autres suppose la possibilité de délaisser un peu de soi, pour accepter un peu plus de l'autre. Aussi l'ouverture d'esprit face à l'autre risque de s'élargir d'autant plus qu'il parviendra à « séduire » en révélant dans la différence la « mêmeté » de notre humanité. Selon le questionnement qui nous habite, cet apport de l'autre qui devient aussi révélateur du caractère commun de notre humanité, peut créer la possibilité que nous voulions nous conformer, en partie, à sa vision du monde ou de façon plus intense, à sa manière d'être.

Enfin, des événements contextuels peuvent également stimuler la réflexivité. La question du sens de la vie devient prégnante lorsqu'on est confrontée en même temps à la naissance d'un enfant et à la mort d'un proche. Bien que nous ne concevions pas un tel événement comme une fatalité, mais bien comme une coïncidence, il reste qu'il a engendré une série de réflexions inattendues. Les phénomènes anomiques, tels la souffrance et la mort, perturbent parce qu'ils exposent l'incapacité de nommer l'inacceptable. Ils suscitent un sentiment d'impuissance qui s'explique par l'impossibilité de rendre compte rationnellement de ce qui se passe. En revanche, ils peuvent favoriser l'introspection et la recherche du renouvellement de soi. Parfois, les réactions à de telles expériences peuvent s'avérer radicales (comme dans le cas de certaines conversions). L'adoption d'un ordre significatif différent, permet, dans certains cas, de ranger et de légitimer les expériences troublantes en fonction d'une nouvelle structure d'appréhension. Elles peuvent aussi inciter à entreprendre des recherches sur la condition humaine en vue de les rendre cohérentes. C'est à cette dernière proposition que je m'identifie présentement et qui explique mon retour aux études.

Je pense que si mon éducation chez les Sœurs a développé une habitude à l'introspection et à la communication intrapersonnelle, mon échange culturel au Mexique m'a fait découvrir, par le biais de la communication interpersonnelle et interculturelle, l'autre, avec ses différences et ses similitudes. Mes études actuelles me permettent d'éclaircir comment la communication intervient chez l'humain dans la construction du sens. Toutefois, si l'interprétation du sens de ces diverses expériences passées et présentes oriente la construction de ma « réalité » actuelle, cette prise de conscience s'effectue aussi en considération de l'interrelation avec l'extériorité. Par exemple, je ne peux faire abstraction du contexte d'ouverture sociale dans lequel j'ai grandi, situation qui a contribué à me distancier du mode de conscientisation collective imposée par l'Église. Nous en déduisons donc, tout comme Jean-François Bayart, que la culture ne constitue pas un corpus de représentations stables dans le temps, un 
corpus refermé sur lui-même, mais un ensemble de codes sociaux qui se transforment en fonction de la façon dont les êtres humains construisent leur « réalité ». Le changement semble donc un facteur qui module considérablement la réflexion et l'action des humains dans la modernité. ${ }^{117}$

\section{2-4.3 Interprétation du changement contextuel dans les pratiques culturelles}

\section{Retour à la table des matières}

Ainsi les acteurs sociaux qui ne sont plus contraints à respecter les normes traditionnelles, mais adhèrent à des ententes collectives établies sur la base du contrat, peuvent s'investir avec plus de liberté dans la dialectique de leurs relations. Ils peuvent gérer, orienter et s'approprier avec plus de latitude les transformations des pratiques culturelles. Même si le poids des normes sociales devient moins contraignant, le traitement du changement va osciller entre deux pratiques: l'une va continuer à s'exercer de façon à conserver le poids de la permanence, en mettant par exemple l'accent sur les rituels et les gestes quotidiens, tandis que l'autre cherchera à souligner l'apport du changement, des irruptions, des cas de déviances ou de création. ${ }^{118}$

Les pratiques culturelles modernes consistent en des combinatoires d'opérations dont on ne peut seulement retenir l'aspect de transmission et de reproduction. Elles reflètent à la fois des stratégies de fermeture identitaire, de situations d'indécision identitaire ou d'élargissement culturel et des dynamiques d'homogénéisation et «d'hétérogénéisation»stimulée par la compétitivité entre groupes, régions ou nations. ${ }^{119}$ En guise d'exemples, il suffit de penser aux mutations sociales provoquées par la colonisation ou la décolonisation de régions sous le contrôle d'empires et aux stratégies des élites, parfois en mal d'intégration, qui veulent consolider leur pouvoir et leur richesse. La perpétuation des stratégies identitaires dépend de la gratification à les reproduire ou à innover.

Cette évaluation stimule les acteurs sociaux à négocier constamment leurs appartenances et leurs alliances sociales. Leurs identifications multiples et relatives contribuent à la transformation de la culture. ${ }^{120}$ Chacune de leur identification, que ce soit celles qui concernent leur statut, leur profession, leur classe, leur ethnie ou leur religion, facilitent leur intégration à un groupe dans une des sphères de la société. ${ }^{121}$ Cet

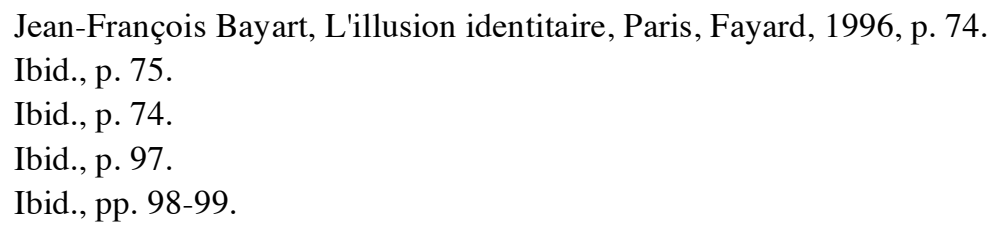


agrégat identitaire qui semble, à première vue, dicter les intérêts des personnes ainsi que leurs projets et leurs passions, oriente l'appréhension des réalités individuelles et les situe par rapport à une pluralité de communautés.

Le sentiment d'appartenance suscité sur la base de ces identifications multiples, ne constitue en fait qu'une croyance opératoire qui donne accès à des réseaux de relations personnelles ainsi qu'à des prises de conscience de communautés variées. ${ }^{122}$ Aussi, pour Bayart, «[...] les identités primordiales « existent» mais en tant que faits de conscience, comme régimes de subjectivité, non en tant que structures. ${ }^{123}$ L'identification ne survient pas tant de l'appartenance à une communauté ou une culture que du rapport aux communautés et cultures avec lesquelles nous sommes en relation.

\section{2-5 Construction des univers communicationnels dans les relations de groupe}

Retour à la table des matières

Lee Thayer a entrevu cette nuance conceptuelle importante. Il développe la nature de ces rapports en accordant beaucoup d'attention à deux relations : celle entre la conscience humaine et son expression sur le plan communicationnel, et celle qui découle de cette interaction. ${ }^{124}$ Selon lui, chaque humain baigne dans un univers d'information qu'il ne peut complètement retenir. Il effectue plutôt une sélection consciente selon qui il est ou encore selon ce qu'il cherche à devenir. Conséquemment, il ne peut que communiquer et négocier que ce dont il est conscient à l'intérieur des réalités communicationnelles qu'il reconnaît. Le monde extérieur façonné par les humains est conçu conceptuellement, au préalable, à l'intérieur de réalités communicationnelles.

Ces dernières qui sont en fait le produit des discours et des univers de chacun ne se définissent pas de façon aléatoire. Ainsi en matière de religion comme dans tout autre sphère d'activités, l'acteur social ne se laisse pas envahir par le discours des autres. Il sélectionne plutôt l'information à laquelle les autres l'exposent, car il ne peut

\footnotetext{
122 Ibid., p. 101.

123 Ibid., p. 101.

124 Lee Thayer, On Communication : Essays in Understanding, Norwood, (New Jersey), Ablex, 1987 , p. $47-51$.
} 
faire totalement abstraction des valeurs et des représentations du monde qu'il a appris au sein des communautés d'interprétation. Lorsqu'il s'identifie à un groupe plus qu'à un autre, il s'associe à un réseau de relations sociales qui coopèrent à l'élaboration de systèmes communicationnels. Et c'est à partir de cette organisation que va se négocier la construction de réalités communicationnelles communes ou exclusives. Ces dernières se maintiennent à l'intérieur de limites tracées par les rôles sociaux, les types de relation et les situations en développement sur le plan social.

En conséquence, la reconnaissance d'un univers communicationnel d'un autre, va également s'effectuer sur la base de certains critères qui vont déterminer l'accès ou non à cette réalité communicationnelle. Thayer considère le principe d'exclusion essentiel à la pratique communicationnelle puisqu'il exige d'exclure l'autre pour parvenir à communiquer avec soi, tout comme il est nécessaire de se distancier de soi pour entrer en communication avec l'autre. ${ }^{125}$ L'incidence de l'exclusion signifie aussi que ceux et celles qui possèdent des univers communicationnels ressemblants, vont exclure les personnes qui ne s'y conforment pas afin de mieux se rassembler. Si l'humain préserve son autonomie par le biais de la médiation qu'il entretient avec luimême, il entretient aussi avec les autres un discours qui s'élabore sur la base de représentations partagées. La communication interpersonnelle favorise ainsi l'établissement de relations sociales qui prennent forme dans l'interdépendance.

Ainsi, communiquer exige, de la part de l'acteur social, qu'il s'oriente dans des univers d'information significative pour lui, où il peut y endosser des réalités qui lui apparaissent vraies parce qu'il les partage avec d'autres personnes qui les reconnaissent aussi comme telles. Il collabore ainsi à l'intérieur d'un groupe, ou d'une communauté, à l'entreprise sociale dans laquelle il s'est engagé. Respectant les accords tacites qui l'unissent aux autres membres, il s'investit à poursuivre ou encore à remettre en question son engagement. Cette dimension tacite de l'entreprise sociale repose sur des croyances, des valeurs, des attitudes, des orientations, un épistème, une parole, un discours. Elle sert également de trame de fond aux jeux et enjeux idéologiques à travers lesquels se négocie l'expression des réalités communicationnelles peu importe le domaine d'activité (politique, économie, esthétique, religion ou autre). Cette dimension tacite réfère à une façon de faire bien connue que l'on cherche à perpétuer ou établir.

125 Ibid., p. 50. 


\section{2-5.1 Types d'entreprises sociales relatives au cas de conversion}

\section{$\underline{\text { Retour à la table des matières }}$}

Aussi Thayer parle-t-il d'entreprises sociales fermées lorsque cette dimension tacite incluant son objet, ses fins, ses règles de base ainsi que ses moyens sont manifestes. De telles entreprises traitent d'objets variés, tels que l'amour, le mariage et même la politique internationale. Elles peuvent devenir des institutions si elles sont reconnues comme telles. À l'opposé, on retrouve aussi des systèmes sociaux ouverts dont l'objet et la finalité ne sont pas vraiment désirés, où les règles et les moyens sont plutôt improvisés. Si les premières structurent la société, les secondes la déstabilisent parce que, contrairement aux systèmes fermés qui cherchent à vouloir se préserver, les systèmes ouverts, en faisant usage de créativité, engendrent des bouleversements.

Prenant en considération ces concepts, la conversion religieuse nous apparaît comme une transition entre deux entreprises sociales selon une des trois possibilités suivantes, soit :

1) entre un système fermé et un autre système fermé ;

2) entre un système fermé et un système ouvert, et vice-versa ;

3) ou encore entre un système ouvert et un autre système ouvert.

Le premier type de conversion fait référence aux personnes qui transitent entre deux traditions religieuses éloignées et qui, plus tard, en viennent à adhérer à un univers communicationnel au détriment d'un autre. Dans certains cas, il se peut que ce soit les proches, membres du groupe religieux, qui ne tolèrent pas le choix de l'entredeux. Dans d'autres situations, il est possible que les convertis s'imposent eux-mêmes une telle contrainte. Parfois aussi, la personne ne change pas vraiment d'épistème, mais souhaite tout simplement joindre les rangs d'un nouveau groupe et partager avec eux leurs croyances. Par exemple, un chrétien protestant d'une secte presbytérienne pourrait décider de s'associer à une congrégation luthérienne. Ils conserveraient, somme toute, les mêmes pratiques, puisqu'il s'agit d'une dénomination chrétienne proche, mais les membres du groupe ne seraient pas les mêmes.

Le deuxième ensemble de conversions se retrouve chez les personnes qui délaissent une pratique religieuse bien encadrée afin de se donner une plus grande liberté d'interprétation. Par exemple, dans l'islam, l'adhésion à foi musulmane n'exige pas du 
Chrétien ou du Juif qu'il renonce à sa religion d'origine. ${ }^{126}$ L'exégèse islamique demande plutôt de reconnaître ces religions. ${ }^{127}$ On retrouve donc parmi les cas de conversion, des adeptes qui vont préférer élargir le schème de leurs représentations au domaine de la foi monothéiste, plutôt que de se restreindre à une religion spécifique. Ces personnes adhèrent ainsi à l'islam tout en préservant certaines de leurs croyances initiales. D'autres, encore, vont procéder sensiblement de la même façon non pas par choix, mais parce qu'elles sont soumises au poids de la contrainte. Elles vont ainsi emprunter, juxtaposer ou synthétiser, des éléments des représentations religieuses conflictuelles, de façon à créer un réagencement de leurs croyances leur permettant de se sentir plus à l'aise. ${ }^{128}$ Cette opération qui est désignée par le terme syncrétisme se produit souvent lorsqu'il y a imposition d'une nouvelle religion dans un contexte de guerre ou de colonisation. Toujours suivant la prémisse que certains convertis vont passer, volontairement ou non, d'un cadre d'interprétation stricte à un autre plus large, on retrouve, quand même, des cas de personnes qui renonceraient à adopter une nouvelle religion. Ces personnes ne chercheraient plus à intégrer un nouveau groupe religieux parce qu'elles considéreraient désormais, que toutes les doctrines religieuses s'équivalent. ${ }^{129}$ Par ailleurs, la possibilité que des personnes veuillent délaisser un cadre interprétatif large pour joindre les rangs d'un groupe partageant une vision du monde bien circonscrite existe également.

Enfin, on retrouve un dernier ensemble d'adhérents ; ceux qui effectuent des recherches dans le domaine des représentations religieuses sans jamais se restreindre à un cadre interprétatif bien établi. Dans bien des cas, ces personnes sont issues de mariages mixtes et vont, par conséquent, adopter un conditionnement moins définitif par rapport à la pratique religieuse. Aussi, vont-elles souvent vouloir reproduire cette approche sur le plan religieux. ${ }^{130}$ Pour elles, il n'y a pas de vérités absolues. Si elles disent parfois s'identifier à une religion précise, toutes ne veulent pas endosser une dénomination particulière dans son ensemble. Elles adhèrent plutôt à un éclectisme spirituel significatif pour elles. Elles veulent continuer à penser par elles-mêmes et choisissent, par conséquent, des groupes religieux où ils peuvent exercer une telle ouverture d'esprit. Selon que les personnes adhèrent à des systèmes fermés ou ouverts en matière de religion, elles vont se conformer à un ensemble bien défini de croyances et de pratiques, ou encore s'investir dans une démarche spirituelle où elles peuvent exercer une plus grande liberté quant au sens de cette expérience.

126 Pierre Assouline, Les nouveaux convertis : Enquête sur des chrétiens, des juifs et des musulmans pas comme les autres, Paris, Gallimard, 1992, p. 223.

127 Selon l'interprétation doctrinale du principe coranique «point de contrainte en matière de religion », ce verset signifie qu'il faut préserver la liberté religieuse des gens du Livre. Voir sur ce sujet, Stefano Allievi, Les convertis à l'islam: Les nouveaux musulmans d'Europe, Paris, L'Harmattan, 1998, p. 44.

128 Mac Cana, Proinsias, Michel Meslin, Collège des Irlandais (Paris), Royal Irish Academy, Rencontres de religions : Actes du colloque du Collège des Irlandais / sous les auspices de l'Académie royale irlandaise (juin 1981), Paris, Les belles lettres, 1986, p. 23.

129 Ali Köse, «The Journey from the Secular to the Sacred : Experience of Native British Converts to Islam », Social Compass, 46(3), 1999, pp. 303-308.

130 John Leland, « Searching for a Holy Spirit», Newsweek, May 8, 2000, p. 62. 


\section{2-5.2 Particularisme et universalisme des réalités communicationnelles}

\section{$\underline{\text { Retour à la table des matières }}$}

Les « réalités mutuelles » qui sont ainsi créées à l'intérieur de ces entreprises sociales par les discussions, tant amicales, qu'officielles, ont une fonction organisatrice autant au niveau de l'esprit que du groupe. Si l'acteur social consent à s'engager dans ces réalités communicationnelles, c'est parce qu'il accepte l'accord tacite qui unit l'ensemble des membres d'un groupe et qu'il reconnaît et partage la façon dont ces personnes perçoivent et rendent compte de leur « réalité » commune. De plus, pour les croyants, l'implication à l'intérieur des institutions religieuses comporte des avantages. Elle procure à ces acteurs sociaux un pouvoir de visibilité et de négociation lors des débats qui surgissent à l'intérieur de l'arène sociale. Elle fournit aussi une tribune de crédibilité pour défendre des intérêts propres et faire connaître une vision du monde en relation aux enjeux de l'heure.

Mais, si l'appartenance à une institution comporte une fonction organisatrice privilégiée, elle peut également conduire à la méconnaissance des autres. La prise de position initiale des évêques canadiens, se déclarant hostiles aux unions des conjoints de même sexe, constitue un bon exemple. L'échange, pour qu'il soit fructueux, exige le respect. Lorsqu'on projette son idéal sur l'autre parce qu'on veut l'assimiler à nos convictions ou qu'on lui est opposé ou indifférent parce qu'on croit à la supériorité de nos croyances, on risque de le méjuger et même de l'ignorer. ${ }^{131}$ Il en va de même si on l'idéalise sans pour autant lui reconnaître son identité propre. La connaissance des autres ne peut se réduire à un moyen pour parler de soi, en bien ou en mal. ${ }^{132} \mathrm{Au}$ contraire, elle incite au dialogue et à l'acceptation de discours autres. Elle réside dans des jugements différenciés qui s'expriment tantôt dans des critères de convenance locale, tantôt en fonction d'une morale universelle. En d'autres termes, l'interaction avec l'autre peut, selon les intérêts de l'acteur en relation avec son environnement immédiat, confirmer le sens de l'interprétation de la personne. Mais cette relation, audelà des apparences, peut aussi contribuer à la connaissance de soi par les valeurs humaines qu'elle révèle. Ainsi l'appréciation des différences peut conduire à l'affirmation de l'identité, non pas au détriment de l'autre, mais selon une perspective d'appréhension plus universaliste.

131 Ces jugements critiques de Todorov sont inspirés des propos de Montaigne et de Montesquieu. Si Montaigne analyse l'autre à partir de ses propres critères de jugement, Montesquieu utilise des considérations plus universelles. Ce dernier prend aussi en considération la relation entre contexte et culture dans son analyse. Tzvetan Todorov, Les morales de l'histoire, Paris, Hachette, 1991, pp. 79-80.

132 Ibid., p. 96. 
La communication humaine ne constitue donc pas une opération simpliste, facile à comprendre ; elle nécessite de l'acteur qu'il croit, qu'il accepte, mais aussi qu'il comprenne l'autre. Si l'être humain a besoin de légitimer son interprétation du monde afin de se comprendre lui-même par rapport aux autres, il a également besoin des autres pour adapter son interprétation du changement.

Les réseaux de coerséduction pendant l'enfance ainsi que les communautés épistémiques auxquels on s'intègre éventuellement remplissent cette fonction ; elles valident des visions du monde par rapport à d'autres. L'élaboration de croyances, d'idéologies, de standards esthétiques, tout comme l'assignation de valeurs morales, constituent en fin de compte des produits de l'intercommunication humaine qui se maintiennent dans le respect d'accords tacites. Lorsque les accords tacites qui unissent les membres d'une organisation sont remis en question des possibilités de conflit émergent. Des problèmes de communication et d'interprétation surgissent. Par exemple, les moyens utilisés pour rencontrer les fins du groupe peuvent devenir inacceptables moralement.

En cas de litige sur l'interprétation des moyens à entreprendre pour adapter la vision du monde d'une organisation reconnue aux développements récents concernant une issue à trouver, l'exclusion d'un membre du groupe peut survenir. L'exclusion d'un acteur social tout comme son inclusion à un autre groupe, sont attribuables en grande partie au seuil de sensibilité de la personne, son imagination, ainsi qu'à sa capacité de décoder ou non les discours inculqués ou recherchés.

\section{2-5.3 Conscience des accords tacites et élaboration des univers communicationnels}

\section{$\underline{\text { Retour à la table des matières }}$}

Nous avons démontré, jusqu'à présent, comment l'acteur social s'engage dans la création et le maintien de ses univers communicationnels afin d'adapter sa vision du monde à celle des autres. On peut toutefois se demander s'il est toujours conscient du rapport qu'il entretient avec ce qu'il construit, surtout en matière de religion. Car, dans ce domaine plus que dans tout autre, l'acteur social a tendance, sur le plan communicationnel, à produire cette « réalité » tout en niant son implication. Aussi parle-t-on de réification, lorsque la conscience appréhende les produits de l'activité humaine comme s'ils étaient non plus des produits humains, mais l'accomplissement de Dieu, de la nature, ou des lois cosmiques. ${ }^{133}$ La réification comme modalité de conscience

133 Peter Berger et Thomas Luckmann, La construction sociale de la réalité, Paris, Méridiens Klincksieck, 1996 p. 123. 
qui serait poussée à l'extrême, pourrait s'apparenter à certaines formes d'intégrisme religieux. Lorsque l'humain ignore sa contribution à la construction de la réalité sociale et qu'il ne s'investit plus dans une relation dialectique où il assume pleinement la responsabilité de son interprétation des faits en relation aux autres et au monde extérieur, il se déshumanise. Mais, que l'acteur social reconnaisse en son propre nom, sa contribution dans l'élaboration de réalités communicationnelles, ou qu'il l'attribue à Dieu, il ne peut les gérer qu'en fonction de la façon dont il les conçoit et les discute.

Ainsi, si l'humain justifie son existence par la façon dont il nomme et explique le monde avec d'autres selon des critères similaires de vérité, il évite le plus souvent de réfléchir sérieusement sur sa condition humaine. En se contentant de vérités relatives, il évite de questionner ce qui devrait être, et reste ainsi dans un état confortable pour ne pas être confronté à la peur de l'inconnu, et à l'angoisse de la créativité. ${ }^{134}$ Georges Bastide, auteur d'un livre sur la conversion spirituelle, écrit ce qui suit à propos de cet alibi qui sert à justifier notre existence ainsi que le désarroi de notre conscience que :

[...] la crainte de ses responsabilités pousse l'homme à se situer ailleurs qu'à la place exacte où éclaterait la honte de sa condition morale. Son orgueil voudrait qu'il fût l'auteur de toutes choses, mais sa faiblesse lui demande de n'y être pour rien. Alors, intervertissant l'ordre authentique, il revendique l'origine de ce qu'il appelle le Bien pour rejeter sur « la force des choses », et parfois même sur un dieu courroucé l'origine de ce qu'il appelle le Mal. Mais comme il ne juge encore de ce bien et de ce mal, que par rapport à sa subjectivité inconsciente, il appelle action ce que la conscience convertie appellera passion et inversement. ${ }^{135}$

C'est pourquoi, pour Bastide, la conversion consiste en l'inversion de cette diversion. Elle consiste en une remise en question profonde des facteurs historiques et culturels par lesquels nous justifions nos états d'être conformément aux normes sociales établies. Mais, comme nous l'avons vu précédemment, elle ne peut être conçue que si elle devient un sujet de discussion pour soi et avec les autres. Pour Thayer, une telle prise de conscience suppose de ne plus concevoir le déroulement de sa vie en fonction des conséquences attribuables aux circonstances, mais plutôt ce qui advient à la suite de nos échanges et de nos conversations. Par conséquent, plus une personne s'engage dans une voie aussi incertaine que celle de la conversion, plus le fait de parler de ce nouvel état de choses devient important et significatif.

134 Lee Thayer, Pieces: Toward a Revisioning of Communication / Life, London, (Eng.), Ablex, 1997 , p. 8.

135 Georges Bastide, La conversion spirituelle, Paris, PUF, pp. 17-18. 


\section{2-6 Conversion des univers communicationnels reliée aux conflits d'interprétation}

$\underline{\text { Retour à la table des matières }}$

Ainsi, communiquer ne consiste pas uniquement en la capacité d'exprimer ses pensées. Chez l'être humain qui fait partie d'une société, cette activité réside plutôt dans cette capacité d'apprendre sans cesse à se comprendre soi-même en relation aux autres, de façon à ce qu'en s'exprimant les autres puissent aussi nous comprendre. ${ }^{136}$ Nous avons l'impression que nous communiquons lorsque nous réglons nos engagements sociaux à l'intérieur de nos multiples réalités communicationnelles. Or, communiquer signifie aussi savoir s'aventurer dans l'entreprise sociale. Savoir entreprendre la prochaine étape relativement à nos engagements tout en respectant les valeurs, les croyances, les règles, les normes qui nous unissent aux autres. Lorsque cette dimension tacite est remise en question, la communication devient un exercice difficilement praticable puisque l'on ne s'entend plus, ni sur les valeurs de base, ni sur les pratiques qui motivent l'adhésion au groupe. Cette situation crée, entre soi et le groupe, un espace propice à la révision des pratiques communes et à la remise en question. Elle peut inciter à considérer une réalité communicationnelle autre allant jusqu'à l'adhésion à un nouveau groupe.

Lorsqu'un constat «d'incom-préhension» se pose, que l'appréhension d'une « réalité » partagée en vient à l'incommunicabilité, cette situation risque de susciter une remise en question profonde de la relation entretenue avec les membres de la communauté d'interprétation. Selon son interprétation de la situation, une personne aux prises avec un tel problème peut vouloir vérifier les fondements de son cadre interprétatif. Peut-être même en venir à la conclusion que sa vision du monde n'arrive plus, conformément à sa position éthique renouvelée, à rendre compte des choses. Si une telle démarche entraîne un changement au niveau des schèmes de représentations, la mise en pratique peut aller jusqu'au changement de mode de vie. Toutefois, si tel est le cas, la personne aura aussi recours à des personnes-ressources qui la guideront dans son cheminement. Le processus par lequel les nouvelles habitudes de vie seront acquises va s'effectuer sensiblement de la même façon que lors de la socialisation primaire. À l'exception que, l'acceptation à se conformer à ces nouveaux comportements ne sera pas inconsciente, comme chez les petits enfants, mais bien consenti volontairement car ce processus oblige aussi à une forme de désapprentissage.

136 Lee Thayer, On Communication : Essays in Understanding, Norwood, (New Jersey), Ablex, 1987, p. VIII. 
De plus, l'acteur social qui est disposé à passer d'une communauté épistémique à une autre, risque de vouloir s'investir dans la construction d'une réalité communicationnelle reconnue, ou en voie de l'être, afin de valider les choix relatifs à sa nouvelle vision du monde. ${ }^{137}$ Dans bien des cas, le discours du groupe qu'il veut délaisser lui apparaît moins persuasif dans les débats qui investissent l'arène sociale. L'organisation entre en compétition avec de nouveaux groupes qui paraissent très actifs dans le contexte social et offrent des possibilités de solutions nouvelles quant aux problèmes et anomalies perçus par le converti.

\section{2-6.1 Crise interprétative relative à l'éthique}

\section{$\underline{\text { Retour à la table des matières }}$}

Si la crise interprétative de l'acteur social se situe sur le plan éthique, il pourra y remédier en autant que les référents éthiques du nouveau groupe puissent résoudre les anomalies perçues précédemment et ainsi s'harmoniser à la position renouvelée de l'acteur social. Les enjeux relatifs à l'éthique sont importants parce qu'ils expliquent les variantes possibles d'adhésion ou de distanciation par rapport à une idéologie, un dogme ou tout autre vision du monde. ${ }^{138}$ Tant que l'acteur social à l'intérieur d'une organisation croit se référer à des valeurs «sûres » dans son interprétation, son désir d'explorer d'autres avenues est pratiquement annihilé puisqu'il se sent appuyé dans sa démarche par la reconnaissance des autres. Cette façon d'appréhender la « réalité » et de lui assigner un sens va créer l'illusion d'une vérité à laquelle correspondra une série d'actions et possiblement un but. Le sentiment illusoire d'être en possession de la vérité occulte les interprétations contradictoires. Toutefois, il suffit de penser aux Croisades ou à l'instauration des camps de « réformation » marxistes ou Maoïstes, destinés à convertir les contestataires du régime politique, pour concevoir que toutes fins n'en justifient pas les moyens. Croire dans l'atteinte de fins justes sans discernement pour les moyens d'y arriver, peut conduire à l'imposition d'une vision du monde qui nie la liberté de ceux qui n'y souscrivent pas.

Tant sur le plan religieux que dans les autres sphères d'activités, on ne peut ignorer que la volonté d'imposer le « bien » puisse conduire au «mal ». Tout comme notre insouciance de la condition des autres peut également démontrer notre irresponsabili-

137 Le phénomène de l'islamisation dans le contexte de la modernité constitue un bon exemple d'une réalité communicationnelle en développement.

138 Les différentes conceptions de l'éthique, que nous mentionnons ici, ont été initialement développées par Max Weber. Pour notre part, nous nous référons sur ce sujet à Eugène Enriquez qui les a reprises dans son livre : Les jeux du pouvoir et du désir dans l'entreprise, Paris, Desclée de Brouwer, 1997, pp. 129-137. 
té. 139 Ce qui prouve qu'une éthique de convictions centrée sur elle-même, qui néglige l'apport de la discussion et de la confrontation avec d'autres points de vue est limitée, voire répréhensible. Il ne faudrait pas croire que les hommes et les femmes de conviction ignorent totalement l'imputabilité de leurs actions, mais plutôt qu'ils négligent dans le choix de leurs décisions de prendre en considération la volonté du plus grand nombre en fonction du contexte. ${ }^{140}$

L'acteur social qui perçoit une telle situation comme une anomalie, cherchera peut-être à s'en dissocier. Il pourra rechercher un groupe qui construit sa réalité communicationnelle en accordant plus d'importance à ses responsabilités envers les autres. Toutefois, s'il limite son attention à cet aspect, il risque d'être confronté à d'autres difficultés. Car il peut s'avérer ardu d'identifier non seulement la nature exacte de ses responsabilités (familiales, sociales, civiques, etc.) mais aussi leur ordre de priorité selon la mouvance du contexte. Par exemple, un pacifiste américain, suite aux événements du 11 septembre 2001, pourrait soutenir des représailles armées. On comprend donc les difficultés pour l'acteur d'exercer une véritable éthique de la responsabilité qui préserve les convictions initiales sans les « détourner » vers d'autres fins.

Il subsiste, toutefois, une différence d'attitude marquée entre ceux qui s'identifient plus à l'éthique de la conviction qu'à l'éthique de la responsabilité. L'acteur social qui interprète la « réalité » en fonction de l'éthique de la conviction peut ignorer ses responsabilités en invoquant la volonté de Dieu ou celle de l'organisation partisane à laquelle il adhère. Par contre, lorsqu'il accorde plus d'importance à l'éthique de la responsabilité, il ne s'aliène pas les conséquences de ses actes. Qu'il croit en Dieu ou en l'Homme-Dieu, il reconnaît alors que les valeurs formelles, telles la générosité, l'altruisme, ou l'équité, sont construites par lui dans l'ordre du sens et qu'il ne peut donc pas simplement les assumer d'un point de vue cognitif. Il doit aussi reconnaître les responsabilités qui y sont associées.

Malheureusement, à l'inverse, l'éthique de la responsabilité peut dériver de son objectif initial en sombrant dans la confusion du relativisme. L'alternative pour pallier ce problème réside, pour J. Habermas, dans l'intersubjectivité. ${ }^{141}$ C'est le propos qu'il développe dans L'éthique de la discussion. Selon lui, chaque citoyen peut très bien se joindre à un groupe à l'intérieur de l'espace public afin d'y discuter et même d'argumenter de façon rationnelle la défense de ses intérêts. Toutefois, souligne Habermas, pour arriver à un consensus, la discussion d'un objet et les conditions de sa

139 Tzvetan Todorov, Mémoire du mal, tentation du bien : Enquête sur le siècle, Paris, Robert Laffont, 2000, p. 335.

140 Eugène Enriquez, Les jeux du pouvoir et du désir dans l'entreprise, Paris, Desclée de Brouwer, 1997, p. 131.

141 C'est également le but de la conversation républicaine appréhendée par J. W. Carey. Voir sur ce sujet, Jay Rosen, «Introduction / «We'll Have That Conversation»: Journalism and Democracy in the Thought of James W. Carey», In James Carey: A Critical Reader, Eve S. Munson and Catherine A. Warren editors, Minneapolis, University of Minnesota Press, 1997, pp. 191-206. 
délibération doivent répondre à des besoins qui sont partagés et énoncés. ${ }^{142}$ Le consensus ne peut être atteint que sur des intérêts universalisables. Les sujets qui participent à de tels échanges doivent s'entendre pour que leurs discours soient sincères et expriment une recherche de vérité. La conscience de points de vue diversifiés au sujet d'un même objet de discussion engendre des concessions de part et d'autre qui tempèrent les convictions de tous. Une telle pratique de la conversation qui met l'emphase sur la personne plutôt que sur ses appartenances, fait de chacun des citoyens égaux, des gens qui vivent ensembles dans un espace commun, un espace public. ${ }^{143}$ Cette façon de procéder permet d'éviter l'exclusion des personnes de l'arène publique sur la base de leurs croyances, spécialement en matière de religion. Dans le sillage de ce mode d'échanges démocratique, des normes sont créées. Elles rendent compte des suites prévisibles aux échanges. Elles délimitent le champ des responsabilités qui doivent être respectées. Ce mode d'organisation sociale nécessite toutefois de la part de tous les participants, qu'ils recherchent la vérité et s'expriment sincèrement.

Habermas souligne, toutefois, qu'il est utopique de penser qu'en procédant de la sorte, il soit toujours possible de formuler des intérêts universels. De plus, parce que cette démarche exige beaucoup d'investissement de soi, il est possible de vouloir limiter ou hâter les discussions afin d'accéder à une résolution plus rapide de l'échange. Ceci aurait toutefois pour conséquences néfastes de générer l'indifférence et de mettre en péril les débats d'idées qui garantissent la démocratie ainsi que l'organisation politique républicaine.

Par conséquent, le schème de valeurs que propose l'éthique de la discussion devient contestable lorsque la motivation à poursuivre jusqu'au bout la démarche discursive est entravée par la morosité, la répétition, la perte d'intérêt. L'intersubjectivité peut alors perdre de vue sa propre finalité. Lorsque l'interaction discursive n'est pas entretenue par la passion du débat d'idées ou la conscience d'urgence qu'impose la mort, le désengagement peut devenir une issue enviable, parce qu'il requiert moins d'effort. Cela compromet le processus discursif.

Néanmoins, la prise en considération de ces lacunes, peut orienter la conscience à la recherche de l'éthique de la finitude. Cette démarche signifie pour les personnes qui s'y investissent de prendre en considération les représentations suivantes :

1) tout d'abord, contrôler ou vaincre la souffrance pour surmonter le narcissisme morbide, l'impuissance, afin de reconnaître les mouvements d'entraînement des pulsions de mort ;

142 Eugène Enriquez, Les jeux du pouvoir et du désir dans l'entreprise, Paris, Desclée de Brouwer, 1997, p. 135.

143 James Carey, «"A Republic, If You Can Keep It” : Liberty and Public Life in the Age of Glasnost», In James Carey: A Critical Reader, Eve S. Munson and Catherine A. Warren editors, Minneapolis, University of Minnesota Press, 1997, pp. 207-227. 
2) ensuite, accorder autant d'importance à la beauté et au plaisir de la pensée qu'à la souffrance, aux limitations, aux peurs et aux passions qui en découlent ;

3) enfin, considérer tout autant l'homogénéisation des structures humaines et leurs perversions que la spontanéité à y contrevenir par des processus d'autonomisation. ${ }^{144}$

Comparativement aux autres formes éthiques, cette dernière accorde beaucoup plus d'importance aux aléas de l'extériorité. Si les impondérables peuvent susciter l'impuissance et les limitations, ils révèlent, en contrepartie, ce qui est méconnu en nous et qui est ravivé par la mort et la souffrance. L'éthique de la finitude en prenant en compte la dualité des êtres, la lumière et l'ombre impartie à chacun, incite à se distancier de ses convictions afin d'éviter les excès de leur défense absolue. Elle invite également à confronter la souffrance ainsi que les limitations propres à chacun, afin de s'investir dans une démarche constamment renouvelée de recherche de vérités. Une telle pratique éthique qui incite à la reconnaissance, sinon au dépassement des limites connues et inconnues dans l'être, pousse à la réalisation d'objectifs. Elle exige également de s'investir avec passion dans une telle quête et d'affirmer sa volonté de s'ouvrir à l'autre, tout comme à soi. Elle demande aussi du jugement pour établir les priorités à assumer, ainsi que de l'imagination pour stimuler la vision d'un idéal valorisant la construction et l'édification du social. ${ }^{145}$ En fait, l'éthique de la finitude constitue un appel à la « sublimation » de l'être et à la sagesse. La conversion spirituelle peut s'inscrire dans une telle perspective d'appréhension morale.

\section{2-7 Conclusion}

\section{$\underline{\text { Retour à la table des matières }}$}

Ainsi, la possibilité qu'une personne envisage de se convertir à une autre vision du monde, peut survenir à la suite d'un conflit entre les membres d'une communauté d'interprétation à propos de divergences éthiques. En d'autres termes, l'interprétation des changements contextuels et les prises de décisions qui en découlent à l'intérieur des groupes peuvent entraîner, pour certains membres, des actions difficilement justifiables sur le plan moral. Cet écart de perception, mis au jour par la rupture de l'accord tacite qui unit les différentes parties qui adhèrent à un groupe, et l'impossibilité de renouveler ce contrat, peut entraîner la désaffiliation d'un ou de plusieurs membres d'une communauté. La possibilité de faire preuve d'un tel détachement sur le plan identitaire tient beaucoup à la réflexivité, c'est-à-dire à cette capacité de réfléchir sur son mode d'appréhension du monde. Cette prise en compte qui

$144 \quad$ Ibid., p. 135.

Ibid., p. 136. 
surgit en relation aux autres fait appel aux facultés télésitiques de l'humain. Mais, si par la communication il est possible de prendre conscience des conditionnements qui orientent notre interprétation du monde, il est beaucoup plus facile de ne pas en tenir compte et de se suffire à soi-même.

De par son conditionnement socialisé, chaque être humain donne une signification à son existence selon le schème d'appréhension qu'il partage initialement avec sa communauté d'appartenance puis avec les membres des communautés de pensée auxquelles il choisira d'adhérer, par la suite. Toutefois, si cette façon d'interpréter le monde conformément au point de vue des autres procure une assurance quant à l'assertion d'une "réalité » particulière, ce schème d'interprétation peut également être relativisé par rapport à d'autres à partir du moment où ce qui est inconnu n'est plus remarqué pour sa différence, mais apprécié pour sa ressemblance. Cela peut se produire dans des circonstances particulières, par exemple, lorsque des changements contextuels vont provoquer des rencontres inattendues révélant des schèmes d'appréhension différenciés. Ou encore lorsque des parents, de par leurs divergences d'opinions, ne transmettent pas vraiment une identité précise, en outre, en matière de religion. Mais l'appréciation de l'autre ne signifie pas pour autant l'adhésion totale à son point de vue. Simplement que l'identité perçoit d'autres façons d'être, d'autres schèmes de référence qui, dans le cas d'un conflit d'interprétation, peuvent en venir à être considérés avec plus d'attention.

Ainsi, la personne qui perçoit des anomalies sur le plan éthique entre le discours et les pratiques du groupe auquel elle adhère, peut en venir à se désolidariser d'une vision collective des choses en remettant en question le sens de l'accord tacite qui l'unissait initialement à tous les membres. Cette personne peut alors chercher à résoudre un tel conflit d'interprétation. L'assimilation d'une nouvelle vision du monde plus conforme aux croyances morales personnelles peut s'effectuer sur une base individuelle ou en relation aux autres, selon les avantages que la personne peut retirer de l'interaction avec les autres. Si la conversion lui permet d'acquérir la même reconnaissance sociale qu'antérieurement, il y a fort à parier que la personne voudra s'investir dans un processus de socialisation secondaire. En procédant de la sorte elle s'assure de faire reconnaître auprès des autres sa vision renouvelée du monde et à partir de laquelle elle entend construire, désormais, sa nouvelle « réalité ». 


\section{Chapitre III Étude de cas : la conversion de Roger Garaudy}

Considérant tout ce qui précède, nous allons rechercher dans les Mémoires et le Testament philosophique de Roger Garaudy, ${ }^{146}$ comment s'est effectuée sa conversion à l'islam. On prendra en compte sa réflexion intrapersonnelle et on examinera ses relations interpersonnelles ainsi que son appréciation du contexte social. Mais pour comprendre de qui nous parlons, esquissons d'emblée le portrait de cet homme.

\section{3-1 Biographie sommaire de Roger Garaudy}

$\underline{\text { Retour à la table des matières }}$

Roger Garaudy est né à Marseille en 1913. De son enfance, il se dit fortement imprégné de l'affection que lui témoignent sa mère et sa grand-mère. Par elles, il apprend que l'amour transforme les êtres qui le portent et ajoute une dimension nouvelle à leur vie ; sa mère garde le souvenir impérissable d'une relation amoureuse impossible tandis que sa grand-mère, catholique dévote, aime Dieu, même si cela

146 Roger Garaudy, Biographie du XXe siècle : Le testament philosophique de Roger Garaudy, Paris, Tougui, 1985, et Mon tour du siècle en solitaire : Mémoires, Paris, Robert Laffont, 1989. 
signifie, pour elle, de se soumettre à sa volonté et d'accepter ses souffrances. ${ }^{147}$ Cette prise de conscience se transforme en une source d'inspiration.

Ainsi si la relation qu'il entretient avec les femmes de sa famille le « séduit », son père athée, revenu aigri de la Première Guerre mondiale, ne l'impressionne guère. De lui, il apprend les affres du premier conflit armé mondial. Grâce à lui, aussi, il prend conscience de l'époque trouble dans laquelle il vit : celle de l'entre-deux guerres, puis de la deuxième grande guerre. Dans un tel contexte, il ne conçoit pas de limiter l'amour dont il se sent porteur, à son entourage immédiat. Plus que cela, il cherche à transposer ce sentiment dans un projet grandiose que l'humanité entière pourra partager. Si la recherche d'un sens à sa vie devient une quête, l'amour, selon ses termes, en sera le guide. ${ }^{148}$ S'il endosse l'identité chrétienne ce n'est pas pour s'y réfugier passivement ; il veut s'investir dans un projet collectif porteur de sens. C'est pourquoi en 1933, à l'âge de vingt ans, il choisit de joindre les rangs du Parti communiste français tout en précisant qu'il est croyant.

Le Parti le mettra à l'épreuve et il sera accepté. Le cercle de ses connaissances s'élargit. En plus des relations qu'il a nouées au sein de groupes religieux et de celles qu'il établit en tant qu'universitaire enseignant en philosophie, il développe un nouveau réseau de relations dans le domaine de la politique - tout d'abord comme militant, puis comme membre du Comité central du Parti (1945), et éventuellement comme député (1945-1958) et sénateur (1959-1962).

Bien que sa carrière politique lui procure une reconnaissance publique, il acquiert également une notoriété considérable en tant qu'intellectuel. Brillant penseur, ses propos trouvent audience non seulement auprès de ceux et celles qui l'entourent dans la sphère politique, mais également auprès de certains intellectuels, membres du clergé, étudiants, lecteurs et personnes qui se questionnent sur le sens d'une époque ${ }^{149}$ Suivant ce qu'il appelle sa méthodologie de l'initiative historique, il recherche dans les contradictions spécifiques d'une société et d'une époque un projet politique capable de les surmonter. ${ }^{150}$ Pour lui, cette démarche ne saurait faire abstraction des valeurs absolues exposées dans le discours des grandes religions car elles régissent les codes de conduite sur le plan social. De plus, elles suscitent l'éveil de sentiments proprement humanistes, notamment celui de se rendre responsable des autres. Et s'il reconnaît une dimension transcendante au comportement de l'être humain, il convient éga-

147 Roger Garaudy, Mon tour du siècle en solitaire : Mémoires, Paris, Robert Laffont, 1989, p. 1318.

148 Cette quête de sens est présente dans ses deux livres de façon très explicite dès le début ; dans ses Mémoires, à partir de la p. 7, et dans son Testament philosophique, à partir de la p. 11.

149 Garaudy a écrit une quarantaine de livres qu'il a fait publier dans diverses maisons d'édition connues. Parmi les sujets qu'il a traités, on retrouve des questions liées à l'esthétique, la morale, la religion, l'histoire et les problèmes du marxisme, ainsi que deux séries d'essais ; l'une où il cherche à établir des ponts entre les civilisations, l'autre, où il envisage l'invention d'un avenir à visage humain.

150 Roger Garaudy, Mon tour du siècle en solitaire : Mémoires, Paris, Robert Laffont, 1989, p. 34. 
lement de sa capacité de surmonter les aliénations ainsi que les déterminismes. ${ }^{151}$ En termes simples, il reconnaît que l'humain participe aussi à la construction de sa vie.

Or, même si l'explication de cette idée est limitée dans son Testament et ses Mémoires, plusieurs indices nous laissent des pistes pour extrapoler. Ainsi, de ces deux ouvrages nous pouvons apprendre que son identité religieuse catholique s'est construite dans le sillage de l'influence de sa grand-mère maternelle, née à Alger et d'origine maure espagnole. ${ }^{152}$ On y apprend aussi que sa motivation à joindre les rangs de la communauté épistémique marxiste s'inscrit dans le contexte de la désintégration sociale due aux deux grandes guerres. Toutefois, si certains événements relatés nous renseignent sur ce qui revêt de l'importance à ses yeux, sa vision du monde et l'orientation de ses actions, il est plus difficile de saisir le cheminement qui l'a conduit à adhérer à l'islam en 1982.

\section{3-2 Étude de la conversion de Roger Garaudy}

$\underline{\text { Retour à la table des matières }}$

Pour comprendre le processus par lequel il en arrive à vouloir se réaliser autrement en s'intégrant à une communauté de foi autre, nous souhaitons explorer trois pistes. Dans un premier temps, nous allons exposer, à partir des œuvres à l'étude, ce que signifie, pour Roger Garaudy, la notion de transcendance. Plus précisément, comment cette idée phare qui guide sa vie a émergé au contact de personnes qui la partagent, mais aussi auprès de celles qui la questionnent et la mettent en doute. Nous voulons ainsi démontrer comment le discours des autres, même s'il va à l'encontre de nos opinions, contribue plus à consolider nos positions qu'à ébranler nos certitudes identitaires.

Ensuite, dans un deuxième temps, nous expliquerons comment l'attachement identitaire à deux communautés de pensée peut devenir conflictuel en fonction de

151 Roger Garaudy, Biographie du XXe siècle : Le testament philosophique de Roger Garaudy, Paris, Tougui, 1985, p. 131.

152 C'est son grand oncle Edouard, qui l'instruit de la généalogie familiale. De lui, il acquiert un intérêt envers ses origines «étrangères », notamment celle de sa grand-mère maternelle dénommée Maurin. Ainsi, dès son jeune âge Garaudy développe une attirance pour l'autre cet «étranger», qui est aussi Maure et musulman. Nous pensons que cette situation favorise l'ouverture à la différence ainsi que la possibilité de l'affirmation éventuelle de considérations identitaires latentes. Roger Garaudy, Mon tour du siècle en solitaire : Mémoires, Paris, Robert Laffont, 1989, p. 23. 
l'interprétation des changements du contexte social, et finir par susciter un dilemme moral.

Enfin, dans une dernière partie, nous éclaircirons comment de tels conflits interprétatifs sont résolus par la conversion identitaire, ce qui permet au converti d'accéder à un nouveau réseau de personnes qui reconnaissent son discours.

Par ces explications, nous espérons rendre plus tangibles les notions théoriques énoncées dans les chapitres précédents. Nous ne prétendons donc pas offrir une version objective des faits, mais plutôt faire comprendre les présupposés subjectifs à partir desquels l'acteur social construit son expérience de conversion selon sa compréhension du monde. Nous envisageons l'étude de cet objet de recherche en sachant que le processus d'écriture du Testament et des Mémoires de Garaudy est en luimême « biaisé ». Les faits ainsi que les explications que donne l'auteur dans ces deux œuvres ne constituent pas le reflet authentique de son expérience. Il s'agit plutôt d'une interprétation revue et corrigée maintes fois, de ce qu'il juge le plus pertinent pour expliquer l'ensemble de ses comportements. Entre la prise de conscience initiale des faits et leurs interprétations subséquentes, la pensée réfléchissant sur elle-même peut se réexaminer plusieurs fois. Bien que l'introspection procède au quotidien à la révision de l'expérience, la réflexivité permet de revoir avec plus de détachement et de façon plus globale les motifs de interprétation pendant une période de vie. Si la subjectivité d'une telle démarche démontre l'impossibilité d'atteindre la compréhension objective des faits, elle n'empêche pas, toutefois, de stimuler l'accès à la connaissance de l'objet étudié dans ce mémoire. Ainsi, nous allons rechercher, les prémisses philosophiques que Garaudy préconise et avec lesquelles il construit sa vision du monde, afin de saisir ce qui est significatif pour lui.

\section{3-2.1 Fondements de la vision du monde de Garaudy}

\section{$\underline{\text { Retour à la table des matières }}$}

Le nœud de la réflexion de Garaudy, porte sur un projet de vie significatif qu'il veut se donner avec les autres. L'orientation qu'il donne à sa vie peut se résumer à un axiome qui inclut deux termes : la transcendance, c'est-à-dire la dépendance de l'humain à l'égard de valeurs absolues, de Dieu ; et la communauté qu'il conçoit comme le devoir pour chaque individu de se rendre responsable des autres. Or, si cet objectif semble tout à fait compréhensible, il comporte son lot de complications tant du point de vue philosophique, que sur le plan religieux et idéologique. Et c'est ce dont nous entretient Garaudy dans la première moitié de son Testament philosophique. Ses critiques à l'égard de la pensée philosophique et religieuse occidentale, l'aident, nous semble-t-il, à mieux faire comprendre l'actualisation constante de la recherche d'une signification à sa vie en relation aux décisions passées. Cette synthèse qu'il écrit sur 
un ton informel, l'amène, à partir de la page 248, mais surtout aux pages $250-251$, à parler en utilisant le Je ; ce qui lui permet d'évoquer plus personnellement les grandes remises en question identitaires associées aux communautés d'appartenance et épistémiques qu'il a intégrées dans le cours de sa vie. Cette pensée réflexive mérite qu'on l'examine de plus près parce qu'elle permet aussi de mieux saisir la nature des anomalies qui ont conduit Roger Garaudy à être exclus du Parti communiste en 1970 et à se convertir à l'islam en 1982.

En premier lieu, nous allons donner une explication philosophique de la notion de transcendance qui semble correspondre à la pensée de Roger Garaudy, afin de saisir le conflit d'interprétation qui y est lié. La définition du concept de transcendance est complexe et le propos de Garaudy sur ce sujet semble, a priori, contradictoire. Selon sa vision du monde, la philosophie, plus qu'une manière de penser, constitue un mode de vie où la foi n'oppose pas transcendance à immanence - la foi commençant pour lui là où la raison se termine. Quelques prémisses philosophiques seront nécessaires pour comprendre ce que représente ce schème d'appréhension de la vie.

\section{3-2.1.1 À propos des concepts de transcendance et d'immanence ...}

\section{$\underline{\text { Retour à la table des matières }}$}

Tout d'abord, il nous semble que toutes les subtilités auxquelles Garaudy fait référence dans son exposé sur les préoccupations de la philosophie occidentale contemporaine tournent autour du questionnement sur la possibilité qu'a l'humain de s'extirper des conditions qui le définissent pour se construire. En d'autres termes, si dans le contexte de la modernité, les humains ne sont plus soumis à l'imposition d'un sens de la vie qui découle de la tradition, les philosophes du XXe siècle se demandent sur quelle base s'effectuent leurs choix et les décisions qui leur appartiennent. Car, si la possibilité d'exercer des choix existe, elle s'apparente, dans bien des cas, à une illusion parce qu'en fait les humains se sentent contraints de perpétuer ce dans quoi ils ont été engagés socialement et économiquement. ${ }^{153}$ De plus, même si l'humain, contrairement à l'animal peut «s'arracher à tous les codes rigides de l'instinct pour aller sans cesse vers plus de perfection culturelle et morale », cela ne veut pas dire qu'il s'est approprié un code de conduite qui lui permette de remplacer de façon immanente les valeurs religieuses. ${ }^{154}$

Plusieurs penseurs ont réfléchi sur ce problème et essayé d'y répondre en proposant leur vision du monde. Toutefois, comme nous dit Garaudy, chacune des explica-

\footnotetext{
153 Majid Tehranian, «La malédiction de la modernité: dialectique de la modernisation et de la com-munication », Revue Internationale de sciences sociales, Vol. XXXII, 1980, No.2, p. 268.

154 Luc Ferry, L'Homme-Dieu ou le Sens de la vie, Paris, Grasset, 1996, p. 14.
} 
tions philosophiques contemporaines sur ce sujet comporte des failles qui n'ont pas été complètement résolues jusqu'à présent. De même en est-il, sur le plan idéologique, des jugements de valeurs qui fondent les idéaux à partir desquels sont établies les conceptions fonctionnelles de la vie collective. ${ }^{155}$ Ainsi, sur le plan politique, le marxisme considéré par plusieurs de « religion de salut terrestre », n'a pu tracer la voie. Sa réalisation en tant qu'utopie d'un Au-Delà par rapport à la vie présente, n'en était pas moins transcendante. ${ }^{156}$

La pensée scientifique contemporaine n'apporte pas plus de réponse; elle se contente de décrire le monde tel qu'elle l'appréhende plutôt que de le concevoir dans l'idéal. Quant à l'éthique, qui prescrit de lutter contre l'égoïsme en exigeant de se soucier de l'autre et exhorte de préférer le bonheur du plus grand nombre à celui d'un seul, elle défend des valeurs qui transcendent la vie et ne semblent pas complètement imperméables à la pensée religieuse. ${ }^{157}$ Aussi, les valeurs fondamentales modernes n'ont rien d'original, sinon qu'elles sont désormais « pensées à partir de l'homme et non déduites d'une révélation qui le précède et l'englobe. » ${ }^{158}$ L'éthique ne découle plus de Dieu, ni des valeurs formelles, elle émane par-delà le bien et le mal dans l'ordre du sens.

Mais l'idée même d'un sens, renferme la présence d'un sujet. « Par exemple, un panneau indicateur a un sens, non parce qu'il indique une direction, mais parce qu'il a été intentionnellement créé par quelqu'un qui veut communiquer avec nous. » ${ }^{159}$ Le sens est donc lié à une volonté, à cette manifestation subjective, même si elle est inconsciente (comme dans le cas d'un lapsus). À la limite, on pourrait même se demander le sens d'un arbre, mais il nous semble qu'il serait difficile de déduire ce que son créateur cherche à nous dire. "En revanche, on peut se demander quel est le sens d'un mot, d'une remarque, d'une attitude, d'une expression, d'une œuvre d'art, ou de tout autre signe dont on suppose qu'il est l'expression d'une volonté, le signe d'une quelconque personnalité. » 160 Par contre le sens de la mort ainsi que de la souffrance reste insaisissable dans cette perspective, parce qu'il ne découle pas de l'expression d'une volonté connue. On ne peut donc qu'admettre l'impossibilité d'annuler toute dépendance à l'égard de l'extériorité. ${ }^{161}$ Cette constatation explique la position de plusieurs penseurs contemporains qui, tout en revendiquant l'autonomie, c'est-à-dire la capacité de penser par soi-même, n'exclut pas son contraire, l'hétéronomie ou la propension de celui qui pense par le regard de l'autre ; cela en autant que les données externes qui sont assimilées soient soumises à un examen critique et rationnel. L'idée de Dieu n'est désormais plus imposée par la tradition, mais surgit à travers le visage

\footnotetext{
155 Fernand Dumont, Les idéologies, Paris, PUF, 1974, p. 16.

156 Luc Ferry, L'Homme-Dieu ou le Sens de la vie, Paris, Grasset, 1996, pp. 21-24.

157 Ibid., p. 40-43.

158 Luc Ferry, L'Homme-Dieu ou le Sens de la vie, Paris, Grasset, 1996, p. 43.

159 Ibid., p. 34.

160 Ibid., p. 35.

161 Ibid., pp. 47-48.
} 
de l'autre qui nous révèle à nous-mêmes. ${ }^{162}$ La prise en compte de ces enjeux philosophiques nous semble guider le projet de vie conçue par Roger Garaudy.

\section{3-2.1.2 Construction de la problématique philosophique de Roger Garaudy}

$\underline{\text { Retour à la table des matières }}$

Ce questionnement explique, entre autres, la lutte qu'il dit avoir menée tout au long de sa vie pour faire reconnaître la dimension transcendante de l'homme. Pour lui, l'humain qui se suffit à lui-même, c'est-à-dire qui ne reconnaît pas sa dépendance à l'égard de valeurs absolues ainsi que sa responsabilité envers la communauté et ses membres, est voué aux pires affrontements. C'est ce qui motive initialement son adhésion au christianisme, puis au marxisme, et par la suite, à l'islam. À l'heure des bilans, il admet toutefois, que la pratique du pouvoir dans chacune de ces communautés crée une confusion entre les fins et les moyens, qui engendre l'abus. Cette prise de conscience ne l'empêche pas pour autant de croire à l'expression d'un dynamisme créateur, qui puisse susciter un projet de vie planétaire nous épargnant de notre propre destruction.

Nous avons tenu à souligner ces prémisses philosophiques parce qu'elles facilitent, selon nous, la compréhension du discours intérieur, au centre des préoccupations de Roger Garaudy. À tout le moins, elles nous permettent de saisir pourquoi il reconnaît les valeurs absolues défendues par la religion catholique, même s'il ne conçoit pas cette vision de la transcendance uniquement en termes d'extériorité. Sa foi n'oppose pas immanence à transcendance. Selon lui, les comportements humains ne sont pas uniquement déterminés par l'autorité divine ; l'humain peut également devenir ce qu'il entend être. Il s'explique ainsi :

1933, c'est l'année où Hitler accède au pouvoir. Le fascisme italien est à son apogée et Mussolini va bientôt envahir l'Éthiopie.

Jeté dans cet univers convulsif, et le cœur plein d'orages [...] je choisis de devenir chrétien.

[...] Chrétien, dans un monde de l'absurde, pour donner un sens à ma vie.

[...] Marxiste, dans un monde livré à la violence, pour donner une efficacité à mon action.

Pendant un tiers de siècle, j'ai tenté, au risque d'être écartelé, de tenir les deux bouts de la chaîne : le marxisme n'était pas pour moi une idéologie ou une vision du monde, mais une méthodologie de l'initiative historique, c'est-à-dire à la fois l'art et la science d'analyser les contradictions majeures d'une époque et d'une société, et, à partir de cette entreprise de conscience, découvrir le projet capable de les surmonter.

162 Ibid., p. 52. 
Entre la foi qui donnait un sens à la vie, et une méthode qui donnait une efficacité à l'action, je ne voyais pas d'antagonisme, mais au contraire, une complémentarité. ${ }^{163}$

Ce schème de pensée avec lequel Garaudy oriente son action s'est construit, comme nous l'avons déjà mentionné, dans la reconnaissance de discours auxquels il a été sensibilisé pendant son enfance et qui ont contribué au façonnement de son identité. Aussi, avons-nous fait le constat, précédemment, de ce qui, sur le plan familial, semblait constituer les prémisses d'un tel assemblage d'idées. C'est ainsi que nous avons remarqué que, dans le contexte d'une époque trouble, son identification chrétienne procède en réaction à celle de ses parents athées, mais en continuité avec les croyances de sa grand-mère. ${ }^{164}$ Nous avons aussi noté que, sa conception de la transcendance va être revisitée et aménagée en fonction des rapports coerséductifs qui vont l'unir à d'autres personnes dans le cours de sa vie. Ces rapports lui permettront d'ailleurs de reconnaître des considérations philosophiques et intellectuelles différentes.

Comme nous l'avons expliqué précédemment, les individus s'associent aux personnes qui semblent partager les mêmes représentations qu'eux. En procédant de la sorte, ils s'engagent dans des entreprises sociales où ils poursuivent des objectifs qui confortent, dans bien des cas, leur identité personnelle.

Les codes ainsi partagés sont significatifs pour eux-mêmes et pour les autres dans la mesure où ils sont reconnus mutuellement. Aussi, plus les membres d'un groupe se côtoient, plus ils partagent leurs idées et leurs sentiments, et plus il devient possible pour eux d'accéder aux représentations des autres, voire à partager des valeurs ainsi que des pratiques similaires. La prise en compte d'une idée, comme nôtre, témoigne de la reconnaissance du discours de l'autre et de la confiance qu'on lui témoigne. Évidemment cette appréciation de l'autre peut être raffinée par la réflexion personnelle ainsi que par l'apport de lectures et de rencontres subséquentes significatives.

Par exemple, de Garaudy on apprend qu'il en vient à concevoir une vision de la transcendance qui inclut l'immanence lors d'une conférence prononcée par Maurice Blondel. Cette vision est aussi alimentée par l'influence de Milaine, une amie qui deviendra son épouse. De la thèse de Blondel, condamnée par l'Église pour immanentisme, Garaudy mentionne que lui et Milaine passent les rares exemplaires de ce document en cachette. ${ }^{165}$

Garaudy parle aussi du département de philosophie de la Faculté à Aix comme d'une «véritable école (chapelle), de piété philosophique où «Milaine est l'expression la plus raffinée et la plus chaleureuse ». Le mémoire de cette dernière sur

163 Roger Garaudy, Biographie du XXe siècle : Le testament philosophique de Roger Garaudy, Paris, Tougui, 1985, p. 250.

164 Ibid., p. 250.

165 Roger Garaudy, Mon tour du siècle en solitaire : Mémoires, Paris, Robert Laffont, 1989, pp. 4142. 
«La Communion spirituelle » s'inscrit d'ailleurs dans la pensée de Blondel et de l'école d'Aix. Et c'est en continuité avec cette ligne de pensée, que Garaudy entend suivre le sillage mystique de ce dernier qui constitue, selon ses mots, un des «tisons » de sa vie. Bien que certaines idées développées par Blondel le séduisent au point qu'elles émaillent son discours, ${ }^{166}$ l'apport de la relation affective et intellectuelle avec Milaine, sa première épouse, n'est certainement pas négligeable dans la reconnaissance et l'appréciation du discours de Blondel. ${ }^{167}$

Une suite d'idées en entraînant une autre, c'est aussi en prenant compte du concept de finalité de Blondel que Garaudy entreprend, dans la même période de sa vie, la lecture des œuvres de Marx. De cet auteur, il découvre la science et la technique de l'efficacité pour accomplir, dans l'action, sa recherche personnelle de significations. Cette lecture semble l'inciter à joindre les rangs du Parti communiste français en 1933. Sa démarche dans cette organisation sera aussi appuyée pendant 37 ans par Maurice Thorez, secrétaire général du Parti communiste français. Garaudy dit d'ailleurs à son propos : "Jusqu'à sa mort, Maurice a gardé sa main sur moi, me faisant accéder jusqu'au sommet de la direction du Parti et me protégeant de tous les sectarismes. » ${ }^{168}$ Ici aussi, on ne peut nier le jeu d'influences entre ces deux personnes et les répercussions qui en ont découlé sur le plan pratique.

Enfin, c'est la pensée de Kierkegaard qui complète la trame de fond de la carte avec laquelle il appréhende et interprète son monde. De cet auteur, il retient «l'insuffisance de nos morales et de nos logiques » et, par conséquent, la nécessité de relativiser les assertions à propos de l'avoir, du savoir et du pouvoir. ${ }^{169}$ C'est cette prise en compte qui constitue, à notre avis, l'élément le plus déstabilisateur sur le plan identitaire. En effet, elle invite à se remettre en question, à se distancier de soi et à s'ouvrir à d'autres possibilités d'être. Kierkegaard propose de renoncer à nos petites raisons d'être, à nos volontés de croissance et de puissance, sources d'affrontement, et suggère plutôt de s'investir dans un dessein plus universel qui tienne compte des valeurs absolues. Si nous ne pouvons repérer le nom d'un proche de Garaudy associé à la pensée de cet auteur, nous estimons que le réseau de relations universitaires qu'il fréquente ainsi que les jeunes pasteurs et étudiants en théologie protestante du Cercle évangélique où il loge, contribuent à soutenir l'appréciation d'un tel auteur.

On remarque que Garaudy construit, par lui-même, un schème d'appréhension de la « réalité » qui découle principalement de la pensée de Blondel, Marx et Kierkegaard. Mais il semble aussi influencé par les gens de son entourage qui évoquent ces

166 Entre autres, l'expérience d'une foi dans l'action motivée par la raison et la nécessité de trouver le sens et la fin de toute action et de toute vie. Pour de plus amples explications voir, Ibid., pp. 138-142.

167 Bien qu'il la quittera pour la guerre sans vraiment revenir à elle par la suite, Milaine sera la première personne qu'il ira retrouver suite à son exclusion du Parti communiste français en 1970.

168 Roger Garaudy, Mon tour du siècle en solitaire : Mémoires, Paris, Robert Laffont, 1989, p. 38.

169 Ibid., p. 41. 
auteurs, discutent de leurs propos et cherchent peut-être à les intégrer à leur vie. De plus, ces échanges d'idées ne sont pas étrangers à ce qui se passe dans l'extériorité, mais se situe dans la mouvance intellectuelle d'une époque qui veut remédier aux souvenirs pénibles de la Première Guerre mondiale; dans un tel contexte, l'idéal du Parti communiste correspond aux attentes de plusieurs.

L'engouement pour cette organisation va en faire une véritable force politique en France. Ainsi, des personnes d'univers d'influences différenciées vont accepter, pour la cause, de s'unir. Ce regroupement ne constituera pas un mouvement monolithique, mais un lieu de rencontres où vont se négocier le sens des représentations. Bien que, de prime abord, la plupart des personnes pensent fixer par l'intermédiaire des mots et du langage le sens des significations, ${ }^{170}$ la possibilité d'un glissement entre les deux reste à tout moment possible ; la représentation ne constitue que l'assertion, à un moment donné, d'un point de vue sur une chose, en regard d'autres possibilités descriptives. Par exemple, les objectifs du Parti constituent une forme d'appropriation de la « réalité » difficilement négociables parce qu'ils sont significatifs, à un moment donné, pour plusieurs personnes. La considération d'autres alternatives risquent d'être évaluées non pertinentes, plutôt que de constituer une issue possible. Tant qu'une personne parvient à maintenir, à l'intérieur d'une communauté de pensée, l'impression de se référer à des représentations similaires à celles des autres, elle risque de maintenir un discours conforme à celui des autres.

C'est ce qui semble se passer chez Garaudy, du moins, pendant une bonne période de sa vie. Car, même s'il se retrouve constamment en situation de se faire influencer par les propos des membres des communautés épistémiques auxquelles il adhère, il n'en va pas de soi assurément. Il reste plutôt fidèle aux schèmes de représentations qui fondent sa vision du monde. L'expression la plus révélatrice de cette idée, se retrouve, selon nous, dans le débat d'idées opposant Garaudy à Sartre et qui va durer dix ans.

\section{3-2.1.3 Deux visions opposées du monde}

Retour à la table des matières

Si ces deux intellectuels vont partager, pendant quelque temps, une passion commune pour le communisme, les prémisses à partir desquelles ils établissent chacun leur vision du monde sont irréconciliables. Pourtant, on pourrait croire que toute personne soumise au discours d'un intellectuel aussi chevronné que Sartre finirait par succomber à son influence. Bien que, dans les deux œuvres à l'étude dans ce mémoire, on puisse relever que Garaudy au terme de leurs séries d'échanges se sent for-

170 François Laplantine, Je, nous et les autres : Être humain au-delà des appartenances, Paris, Le Pommier-Fayard, 1999, pp. 89-106. 
tement ébranlé par son opposant, ultimement, il va réitérer le discours qui évoque sa vision du monde tout en le nuançant davantage. Afin de démontrer notre propos, nous allons d'abord expliquer la nature du débat opposant les deux penseurs pour saisir, ensuite, comment s'exerce la joute persuasive entre l'un et l'autre et, ultimement, leur incompatibilité spirituelle.

Ainsi, en relation avec ce que nous avons déjà dit à propos du dilemme opposant les concepts de transcendance et d'immanence, Sartre va défendre, une position radicalement anti-déterministe. Selon lui, il faut renoncer à se concevoir dans l'ordre de l'univers comme des entités dont la place est déjà fixée par un Dieu qui détermine le sens de cette occurrence. Plutôt que de se restreindre à vivre une « réalité » conforme à nos attachements identitaires (religieux, ethniques, économiques, etc.), il propose de s'en distancier pour mieux explorer les potentialités de notre pouvoir télésitique. Sartre ne se dit pas contre Dieu; il refuse simplement les images créées à son sujet par les hommes.

Garaudy reconnait également cette possibilité chez l'humain de surmonter ses conditionnements pour constituer sa propre histoire. Toutefois, il ne peut s'astreindre à ne pas considérer les entraves qu'impose le respect de la liberté des autres, à l'expression d'un tel projet. S'il conçoit, comme Sartre, «que nous ne sommes pas devant une finalité toute faite en dehors de nous », il propose de prendre connaissance de nos déterminismes non pas comme des dogmes, mais simplement comme des postulats. ${ }^{171}$ Mais cette subtilité conceptuelle ne semble pas le satisfaire entièrement. Comment concevoir l'expression de la liberté humaine sans qu'elle soit limitée par des préconceptions, mais aussi sans qu'elle nuise à l'expression de la liberté des uns par rapport aux autres ? Garaudy qui saisit bien la difficulté conceptuelle attachée à cette question, pourrait en venir à choisir la position philosophique de Sartre ou encore se dire agnostique. Dans le doute, il demande plutôt conseil auprès du père Troisfontaines, un membre de son réseau de relations, lors d'une réunion de l'Association Teilhard de Chardin. Garaudy lui dit ne pas remettre en question sa foi en la transcendance, mais avoue vivre cette relation moins comme une présence et une promesse, qu'une absence et une exigence. ${ }^{172}$ Troisfontaines répond amicalement à Garaudy : «[...] et si vous vous interrogiez sur ce qui fonde cette exigence ? Cette conscience de l'absence n'a-t-elle pas besoin d'une présence pour être vécue comme un manque ? »173 Ce questionnement, chez Garaudy, semble traduire un certain désarroi moral. À la suite des discussions avec Sartre, son interprétation du « religieux » paraît lui peser. Néanmoins, c'est la réponse de Troisfontaines, même si elle semble ambiguë, qui vient le conforter.

Nous remarquons ainsi, que devant l'impossibilité d'en arriver à une conclusion sur une question philosophique et religieuse aussi complexe que celle soulevée lors

\footnotetext{
171 Roger Garaudy, Mon tour du siècle en solitaire : Mémoires, Paris, Robert Laffont, 1989, p. 191.

172 Ibid., p. 192.

173 Ibid., p. 192.
} 
des échanges avec Sartre, Garaudy préfère, dans le doute, consulter un membre de son réseau de relations dans lequel il a confiance pour l'éclairer sur le sujet, plutôt que de renoncer à son point de vue. Parce que si tel était le cas, cette conversion remettrait en question toutes les références identitaires à partir desquelles il a construit sa façon d'appréhender le monde. Il n'est pas prêt à vivre un tel revirement de situation. Le choix de la personne à qui il demande conseils est révélateur ; puisqu'il connaît les fondements de sa pensée, pourquoi se référer à lui plutôt qu'un autre ? En soi, la réaction de Garaudy confirme ce que plusieurs chercheurs en communication ont remarqué : l'humain se laisse difficilement entraîner dans l'inconnu en faisant abstraction de toutes les considérations matérielles et idéologiques qui l'ont conditionnées par le passé. Comment appréhender le monde sans faire référence aux points de repères qui ont depuis toujours balisés la carte radar des interprétations ? À tout le moins, il semble que l'incertitude à propos des fondements épistémiques qui oriente l'interprétation de la « réalité » ne constitue pas un critère suffisamment significatif pour susciter la considération d'autres influences. Si Garaudy perçoit mieux les enjeux relatifs aux concepts de transcendance et d'immanence, les convictions à la base de sa vision du monde ne semblent pas encore ébranlées ; l'affrontement avec Sartre, bien qu'il le questionne, ne le détourne pas de son projet humanitaire, pas plus que du sentiment de responsabilité envers les autres qui anime sa réalisation.

Les conditions pouvant inciter à l'acte de conversion ne semblent pas réunies. Du point de vue de la communication intrapersonnelle, l'acte réflexif, même s'il permet à la pensée de se distancier d'elle-même afin de s'auto-évaluer, n'offre pas, toutefois, la possibilité d'appréhender le monde par l'intermédiaire d'un autre schème d'interprétation. Car enfin, pour en arriver à apprécier et même intégrer une autre vision du monde, il faut relativiser ses attachements identitaires, c'est-à-dire renoncer en partie à l'interprétation de ses schèmes de référence pour parvenir à en apprécier une autre.

\section{3-2.2 Détachement identitaire et conflits interpersonnels}

\section{$\underline{\text { Retour à la table des matières }}$}

Afin de faciliter la compréhension de notre propos, nous allons recourir à un exemple : celui de la Malinche. Bien que nous ne sachions pas si ce personnage historique s'est converti religieusement en même temps que culturellement, nous estimons que, la description de la modification de ses rapports interpersonnels nous aident à mieux cerner le processus par lequel se produit le détachement identitaire rendant possible l'acte de conversion. Cet exemple, que nous utilisons afin d'en retirer par analogie des similarités avec le cas Garaudy, servira aussi à étayer notre propos, un peu plus loin, dans ce chapitre. Mais avant d'entrer dans le vif du sujet, il nous faut présenter le personnage. 


\section{3-2.2.1 La conversion de la Malinche}

\section{$\underline{\text { Retour à la table des matières }}$}

La Malinche est une interprète qui a facilité la conquête de l'empire aztèque par Cortés. ${ }^{174}$ Dans sa culture d'origine, elle apprend le nahuatl, la langue des aztèques. Ayant été vendue aux Mayas, elle en vient à parler leur langue ; il en sera de même, plus tard, avec les Espagnols. Comme elle connaît l'usage de plusieurs langues, on l'utilise comme interprète. Toutefois, ce qui est très révélateur, selon nous, c'est qu'elle ne se contente pas de traduire. À la demande des Espagnols, elle interprète non seulement les mots, mais également les comportements, les réactions et les stratégies de Moctezuma, le chef aztèque. Todorov suggère qu'elle effectue cette véritable conversion culturelle en réaction à son ancien statut d'esclave.

Bien sûr, cela n'est sûrement pas étranger à ses motivations d'accéder à de meilleures conditions de vie. Néanmoins, ce qui nous semble encore plus significatif se situe dans l'expérience communicationnelle qu'elle vit, en première instance, auprès des Mayas. En totale immersion dans une culture qui lui est étrangère, elle apprend à reconnaître le particulier de l'autre et l'accès à ce savoir méconnu lui permet, par ricochet, d'accroître sa connaissance de sa propre identité. Cette prise de conscience par la distanciation et la réflexivité bouleverse son intériorité. De par sa condition d'esclave, elle ne peut s'éviter l'effort d'apprendre à connaître l'autre si elle veut survivre. Comme elle ne peut plus appliquer au monde extérieur ses références habituelles pour décoder sa « réalité », elle en vient à considérer le point de vue de l'autre, à prendre conscience des spécificités culturelles des uns par rapport aux autres, mais aussi à apprécier les similitudes qui unissent tous les humains. Sa vision du monde est bouleversée par ces insertions dans des réseaux de cultures différentes.

Elle est toutefois contrainte de revivre cette expérience de l'altérité et du déconditionnement auprès des Espagnols. Toujours impuissante face à sa condition d'esclave, acculturée par son séjour chez les Mayas, la Malinche perd, au fil du temps et au contact des autres, son sentiment d'appartenance à la culture aztèque. Elle fait désormais partie de la grande famille de l'humanité. L'éloignement l'a rendue en partie étrangère à sa propre culture. Pendant son absence, elle n'a pas pu suivre les transformations de la culture aztèque. Aussi, a-t-elle pris ses distances face à la «mêmeté » qui se manifeste dans la stabilité du caractère et du comportement de gens de même culture, et qui facilite les interactions et la communication entre les individus. C'est plutôt «l'ipséité » ou l'Autre en elle, c'est-à-dire tout ce qui lui est

174 Tzvetan Todorov, La conquête de l'Amérique : La question de l'autre, Paris Seuil, 1982, pp. 130-132. 
inconnue d'elle-même, qui se révèle au contact de l'étranger. Cette découverte qui lui permet de se distancier de ses conditionnements identitaires aztèques, lui fait aussi entrevoir les conditionnements culturels autres. La Malinche exploite ainsi certaines croyances largement partagées dans sa culture initiale afin de les mettre au profit des objectifs de conquête des Espagnols. Et plus particulièrement de ceux de Cortés qui, en échange, lui redonne la reconnaissance au sein d'un nouveau réseau de relations.

On peut d'ores et déjà deviner la forte inclinaison de la Malinche pour le capitaine conquérant qui lui offre toute sa considération. Pas surprenant dans ces conditions qu'elle accepte, en échange, de lui faire part de son savoir. Son labeur consiste à exercer une pratique qu'elle connaît bien pour l'avoir expérimentée auprès des Mayas, et qui consiste en la réception active ; c'est-à-dire l'opération de décodage qui, par-delà des mots, rend compte de «l'ordre symbolique » d'une culture, en l'occurrence celle des Aztèques, et qui permet à Cortés de mieux anticiper la réaction de ces derniers. La Malinche réussit à confondre, par son trafic des signes, Moctezuma en lui faisant bien croire que la venue des Espagnols correspond à une prophétie ancienne à propos du retour du dieu Quetzalcoatl. ${ }^{175}$ En manipulant l'information de cette façon, elle transforme le mythe en réalité, suscitant ainsi la peur du chef Aztèque. Elle facilite ainsi continuellement l'action des Espagnols afin d'assurer leur conquête.

Cette situation la met en position de pouvoir. D'une part, l'impossibilité d'intégrer sa société d'origine en tant qu'esclave favorise son alliance avec les Espagnols qui n'hésitent pas à lui offrir un statut particulier en échange des informations précieuses qu'elle leur procure. D'autres part, ces derniers en manque de repères ont absolument besoin d'elle dans leurs relations avec les populations locales. Au plus fort de la conquête, Cortés entretiendra une relation particulière avec elle. Plus tard, elle se mariera à un officier espagnol. Son adhésion à une autre vision du monde sera complétée.

Ce renversement de situation survient grâce à un savoir né de la distanciation avec sa propre culture. Motivée par ses intérêts, elle est entrée en relation avec un environnement humain différent qui l'a incitée à modifier sa perception des choses et à adopter des comportements autres.

Nous notons la présence d'un phénomène similaire dans le parcours de vie de Roger Garaudy. Tout comme la Malinche, Garaudy, en tant qu'intellectuel, en vient à être reconnu comme leader d'opinion. Nous ne pouvons ignorer qu'il s'est investi dans l'écriture de plus de quarante livres. Si ce travail lui procure une certaine notoriété, il le fait également entrer en contact et échanger avec tout un réseau de relations qui adhèrent à ses propos, les questionnent ou encore les critiquent. En tant que professeur, conférencier et représentant du Parti communiste, entre autres, Garaudy est confronté à d'autres penseurs et leaders d'opinion qui ont des sujets de préoccupation similaires au sien (Sartre par exemple). Il fait grand cas des rencontres marquantes, en

175 Ibid., p. 151. 
personne ou par l'intermédiaire des livres, qui ont participé, positivement ou négativement, à la construction de sa vision du monde. ${ }^{176}$ Il signale aussi la contribution des personnes qui ont participé à mettre en perspective ses idées et l'ont amené à se questionner plus profondément. ${ }^{177}$ Mais, ce qu'il souligne avec le plus de conviction, et cela dès l'introduction de ces deux livres, ce sont les problèmes graves d'interprétation vécus à l'intérieur des différentes communautés épistémiques auxquelles il s'est joint dans le cours de sa vie. ${ }^{178}$ Non pas que ces difficultés l'aient conduit à changer radicalement sa façon de voir les choses. Elles l'ont plutôt amené à vouloir réaliser son projet humain selon d'autres modalités, d'autres moyens plus conformes à sa conscience éthique. C'est donc la prise en compte d'un sens lié à une pratique qui satisfait un besoin ou est affecté à un usage qui semble remis en question. 179

\section{3-2.2.2 Décodage et divergences d'interprétation}

\section{$\underline{\text { Retour à la table des matières }}$}

Comme nous l'avons laissé entendre précédemment, les personnes n'assignent un sens aux informations qu'elles appréhendent que dans la mesure où elles peuvent les interpréter selon leur schème de représentations. Si la réalité existe en soi, nous y référons tous par l'intermédiaire du langage auquel nous assignons des valeurs qui s'inscrivent à l'intérieur de visions du monde. Ce partage de réalités communes acquises par coerséduction au sein des communautés d'appartenance et épistémiques, distingue les membres d'une communauté de ceux d'une autre. Bien plus qu'une transcription du réel, les mots sont utilisés pour ériger des conventions entre membres de communautés. Ces ententes en viennent à passer quasi inaperçues tant elles vont de soi. Ces ensembles d'information ou de paradigmes qui régissent les comportements humains dans les différentes sphères d'activité constituent des cartes de réfé-

176 De nos lectures de ces livres nous avons noter les personnes suivantes : le philosophe Maurice Blondel, Marx, Kierkegaard, Rousseau, son mentor au Parti communiste, Maurice Thorez, sa première femme, Milaine, les poètes Aragon et Éluard, l'abbé Pierre, sa deuxième femme, Paulette, Monseigneur Fontenelle, premier chanoine de Saint-Pierre de Rome, Khrouchtchev, Nasser, Ber-nard et Loulou , prêtres ouvriers, Teilhard de Chardin, la danseuse, Martha Graham, Aurelio Peccei, fondateur du Club de Rome, Althusser, Béjart, l'impératrice Farah Dibah, son maître spirituel, le père Chenu, pour ne nommer que ceux là.

177 Nous retenons sur ce sujet : l'entretien avec le Cheik Ibrahim, sa relation avec Staline, le débat avec Sartre, les propos de dom Helder Camara, la confrontation avec Georges Marchais, membre du Parti communiste français.

178 Voir à cet effet, Roger Garaudy, Biographie du XXe siècle : Le testament philosophique de Roger Garaudy, Paris, Tougui, 1985, p. 12. et du même auteur Mon tour du siècle en solitaire : Mémoires, Paris, Robert Laffont, 1989, p. 9.

179 conclusion de ce paragraphe est inspirée des propos de Stuart Hall, « Codage/Décodage », Réseaux, No 68, CNET, 1994, p. 31. 
rence qui facilitent le décodage des informations et qui peuvent aussi stimuler des pratiques sociales spécifiques.

Le théoricien Stuart Hall qui a étudié les pratiques de décodage de téléspectateurs, a remarqué que cet exercice ne s'effectue pas en tout sens. Bien que Hall situe ces opérations de décodage dans des rapports de classe où les récepteurs, négocient ou non, les termes véhiculés par une élite en contrôle des médias de diffusion, nous pensons que son raisonnement peut aussi s'appliquer à la façon dont sont décodés les signes en fonction des adhésions aux communautés d'appartenance et de pensée.

Nous les présentons un peu différemment. Spécifions tout d'abord, que ces opérations s'accomplissent en tenant compte d'un ensemble de références qui crée des rapports de significations selon trois possibilités. Dans la première, le récepteur décode un énoncé suivant les mêmes termes de référence utilisés dans la diffusion par les membres de sa communauté d'interprétation. Dans la seconde, le récepteur bien qu'il comprenne les inflexions littérales du discours de l'autre, le décode en niant ses particularités de façon à réitérer sa vision des choses ; sans ouverture pour l'étrangeté, il reformule son message en fonction de ses présupposés. Enfin, selon la dernière possibilité de décodage, le récepteur négocie les termes de l'échange ; s'il s'adapte en partie aux références de l'autre, il s'oppose également à certains de ses présupposés.

En d'autres termes, l'appréhension des signes lorsqu'elle ne tient pas compte de la relativité identitaire, c'est-à-dire de la prise en compte du regard de l'autre, tend à confirmer des rapports aux choses qui réitère les conventions de sens acquises au sein des réseaux de relations. Cette façon d'assigner des significations peut également se pratiquer par la négative. La personne affirme alors sa vision du monde en rejetant un point de vue différent. Enfin, selon la dernière possibilité et prenant en considération l'expérience de la Malinche, la prise en compte d'un schème d'appréhension autre, par empathie ou au contact de l'autre, relativise l'interprétation; la perception des choses ne va plus de soi, mais devient négociable. Aussi, selon nous, la possibilité de relativiser l'interprétation contribue grandement à la construction du processus de conversion.

Lorsque nous essayons d'appliquer notre interprétation des opérations de décodage au cas Garaudy, nous remarquons évidemment qu'à l'intérieur des communautés épistémiques où il s'est investi, il s'est entouré tout au long de sa vie de gens qui partageaient des idéaux similaires. Par exemple, durant la Seconde Guerre mondiale il devient prisonnier politique, en Algérie, parmi un groupe de militants communistes qui, tout comme lui, se sont fait arrêter puis déporter pour refus d'endosser le Pacte de Munich et plus globalement la politique du Maréchal Pétain. Si cette expression de solidarité traduit en quelque sorte, un décodage similaire de la "réalité », elle constitue aussi une démonstration d'opposition par rapport à d'autres propositions idéologiques. 
En fait, elles représentent l'endos et l'envers d'une même médaille; l'appréciation ou la dénonciation idéologique porte sur la reconnaissance ou non du même idéal. L'idéal « [...] réside dans une conception fonctionnelle de la vie collective » tandis que « l'idéologie est perçue comme un surplus, un ajout par rapport à des mécanismes sociaux qui, au fond, sont censés se suffire à eux-mêmes. » ${ }^{180}$

Évidemment, ces croyances qui agissent aussi comme forces motrices au fonctionnement des organisations, représentent aussi des luttes au niveau des valeurs. Par mesure de simplification des procédures, lorsque l'idéologie repose sur un postulat nettement avoué, on se garde bien d'expliciter constamment le contenu de ces valeurs, et l'on se contente plutôt d'esquisser rapidement les aménagements par lesquels leur manifestation sera rendue possible. Or, sur ce point, Garaudy semble endosser une position plus relativiste. S'il perçoit l'idéologie comme une « réalité » qui influence les hommes et leurs institutions, une force historique qui permet d'anticiper l'avenir et soutenir la volonté de façon rationnelle pour répondre à certaines exigences fonctionnelles, il remarque et souligne, à maintes reprises, un écart grandissant entre la justification des actions et l'image de ses intentions. ${ }^{181}$

Ainsi, il en vient à critiquer abondamment le christianisme et le marxisme. Cette pratique va toutefois le placer en position de négociation constante avec les autres membres des communautés épistémiques auxquelles il adhère. Nous allons expliquer en quoi consiste ces critiques parce que tout en nous permettant de mieux apprécier les considérations éthiques qui ont un sens pour lui, elles font aussi saisir les références qui vont l'opposer éventuellement aux discours des membres des communautés de pensée auxquelles il adhère. Ces éléments nous semblent essentiels pour comprendre la conversion de Roger Garaudy à l'islam.

\section{3-2.2.3 Du catholicisme...}

$\underline{\text { Retour à la table des matières }}$

Ainsi, en relation aux deux communautés épistémiques auxquelles il donne son assentiment, nous allons exposer, en premier lieu, ce qu'il finit par trouver irritant à propos du catholicisme. Bien que Garaudy se montre critique à l'égard de l'interprétation dogmatique que défend l'Église en tant qu'institution, il ne remet pas en cause le christianisme. C'est ce qui ressort de son propos. Plus spécifiquement, il dit avoir lu avec désespoir les deux Encycliques Rerum Novarum et Quadragesimo anno qui définissent la doctrine sociale de l'Église depuis le premier concile du Vatican tenu en 1870. Selon lui, ces documents font état de la volonté de poursuivre la

180 Fernand Dumont, Les idéologies, Paris, PUF, 1974, p. 16.

181 Notre interprétation des propos de Garaudy est inspirée ici de Fernand Dumont. Ibid., p. 20. 
centralisation monarchique du catholicisme entamé depuis le $12 \mathrm{e}$ siècle. L'objectif des directives institutionnelles et idéologiques qui y sont mentionnées, vise à maintenir l'ordre social établi sur la base d'un système de classes hiérarchisées. L'Église octroie ainsi le pouvoir d'appliquer ses normes morales aux princes de droit divin parce qu'elle les considère comme la manifestation de la Divinité créatrice. ${ }^{182}$ Cette vision du monde condamne par conséquent le socialisme ainsi que le libéralisme qui font la promotion de l'État laïc, la liberté de presse et la liberté de conscience. En d'autres termes, les gens doivent accepter leur position dans la hiérarchie sociale, rester soumis aux autorités cléricales et monarchiques et faire preuve d'abnégation en acceptant leur souffrance.

Mais des changements importants dans le contexte social allaient ébranler cette conception du monde. Premièrement le développement du capitalisme va bouleverser la fixité des rapports sociaux. ${ }^{183}$ Ensuite, l'essor du mouvement ouvrier et la création de partis révolutionnaires d'orientation marxiste vont contribuer à la remise en question d'un système économique basé sur la propriété privée des moyens de production qui entretient les divisions hiérarchiques de classe. Cette vague de contestation qui s'attaque aussi aux fondements idéologiques de l'Église va créer une rupture sans précédent avec la conception romaine du plan divin. Pour la première fois dans l'histoire de l'humanité, les humains vont oser se rendre maîtres de la nature et du cours de leur vie. Évidemment ce phénomène aura d'autant plus d'ampleur dans la conscience des gens que l'impact social de l'industrialisation se fera sentir ; l'Europe et l'Amérique latine constitueront des régions plus particulièrement touchées par ce phénomène.

Bien sûr, ce questionnement idéologique qui affecte les comportements religieux ne sera pas interprété par les acteurs sociaux de la même manière qu'il s'agisse de patrons, d'ouvriers ou de membres de clergé. Si pour les uns, le sens des pratiques religieuses signifie de se mettre au service d'une «Majesté » ainsi conçue comme une entité objective, d'autres en viennent à les interpréter beaucoup plus comme une présence subjective du divin qui se manifeste dans l'action pratique et la mise au service des besoins humains. ${ }^{184}$ Ainsi la pratique religieuse se vit, non plus uniformément dans l'attente passive d'un monde meilleur, mais aussi, désormais, comme un investissement de soi à la poursuite d'un idéal. L'individu devient la mesure de toute chose et par conséquent, tout système économique qui compromet la dignité humaine en valorisant l'ambition personnelle aux dépens de l'exercice de la responsabilisation apparaît moralement discutable. ${ }^{185}$

En réaction à cette nouvelle conception de la pratique religieuse, l'Église va faire valoir la nécessité de récompenser la capacité d'initiative des dirigeants dans le pro- 
cessus de production ainsi que l'exigence de prendre en considération la complémentarité des relations humaines; les uns pourvoyant aux incapacités personnelles des autres dans l'atteinte d'objectifs sociaux enviables. Pour l'Église, la prise en compte de tels énoncés qui constituent aussi en soi un but à parfaire, ne peut être envisagée que dans l'aspiration à une totalité représentée par Dieu. L'institution religieuse proclame ainsi une vision du monde où, chaque personne, plutôt que de prioriser uniquement la défense de ses intérêts personnels, se met aussi au service d'autrui afin d'accomplir, par amour, cette réalisation. Ces explications permettent à l'Église de maintenir ses positions tout en se prononçant contre les mouvements révolutionnaires qui veulent abolir le système des classes hiérarchisées.

Toutefois, l'envers d'une telle affirmation communautaire réside dans l'impossibilité, chez l'humain, d'en arriver à accomplir sa vocation individuelle. La position des individus étant déterminée dans cette hiérarchie sociale, ils ne peuvent aspirer à s'accomplir autrement. Aussi, le discours opposé à celui de l'Église va-t-il souligner la nécessité d'altérer les processus sociaux par des luttes collectives afin de parer à l'injustice systématisée. ${ }^{186}$ La foi, dans cette optique, se vit davantage comme un acte de conscience. Mais si certains l'assimilent comme une espérance dans le Christ révolutionnaire, elle reste pour beaucoup d'autres, un moyen de préserver la paix sociale.

C'est à cette mutation inédite des croyances que l'Église est confrontée à partir des années 1960. Et c'est aussi en relation avec ce changement des mentalités que prend forme l'aggiornamento, c'est-à-dire l'adaptation de la tradition de l'Église à la « réalité » contemporaine ${ }^{187}$ lors du concile œcuménique Vatican II tenu entre 1962 et 1965. À la suite de ces assemblées extraordinaires, réunissant le Pape Jean XXIII, (puis Paul VI à partir de 1963), un grand nombre de pères conciliaires (plus de deux mille), ainsi que plusieurs experts, vont se profiler deux courants épiscopaux ; l'un progressiste et l'autre porté vers la tradition.

Les évêques les plus avant-gardistes proviennent principalement du tiers-monde. Ils semblent plus sensibles à l'inhumanité des conditions de vie de leur milieu. Ce qui, sans doute, les rend plus conciliants envers le socialisme et les questions touchant à la démocratisation de l'Église. Parmi eux se trouve dom Helder Camara, un ami fidèle de Garaudy pendant plus de vingt-cinq ans. C'est lui qui va initier Garaudy au courant de pensée de la théologie de la libération qui entend remédier à la souffrance humaine par l'action. ${ }^{188}$ Même si, chacun d'eux œuvre au sein de communautés d'interprétation qui se disent opposées, tous deux semblent endosser des présupposés éthiques similaires. Le pacte qu'ils vont conclure entre eux pour dénoncer les injustices attribuables au système capitaliste ainsi que les perversités du socialisme, en at-

\footnotetext{
186 Ibid., pp. 31-32.

187 Cette définition provient du Petit Robert 1, dictionnaire de la langue française.

188 Roger Garaudy, Mon tour du siècle en solitaire : Mémoires, Paris, Robert Laffont, 1989, pp. 225-226.
} 
teste. ${ }^{189}$ Cette rencontre rend compte également de la problématique majeure à laquelle est confrontée l'Église dans les années 1960. Elle doit se positionner, voire se renouveler par rapport à l'idéologie marxiste qui gagne du terrain.

Bien que l'examen de conscience auquel s'est livré l'Église a permis d'actualiser certaines de ses pratiques, 190 il reste que, sur le fond, la vision hiérarchique entretenue par l'Église n'a pas été remise en question. ${ }^{191}$ Cette constatation parmi d'autres amène Garaudy à qualifier Vatican II de « défaite de l'espérance ». ${ }^{192}$ Et il ajoute :

[...] «L'Église a commencé à s'occuper des faibles lorsqu'ils sont devenus une force». La «doctrine sociale » de Rerum Novarum, venait après la création, en Europe, des syndicats ouvriers, et de la Première internationale socialiste. Elle n'ouvrait pas une voie d'avenir à la classe ouvrière. Au contraire : à une classe ouvrière décidée à se libérer seule de ses misères, de ses humiliations, de ses dominations, si longtemps renforcées par les coalitions pseudo chrétiennes de la «Sainte Alliance» des princes et de leur clergé, et par les condamnations pontificales de tout mouvement de libération et de tout socialisme dans le «Syllabus », elle tentait, en dénonçant non le capitalisme, mais ses «abus », de tracer une voie médiane entre le conservatisme aveugle et une révolution autonome : la voie d'une collaboration avec l'église et l'État, pour tenter de canaliser, sous sa tutelle, un mouvement irrépressible. ${ }^{193}$

Garaudy développe donc une argumentation très critique à l'égard de l'Église catholique. Son rôle d'intellectuel en constante analyse du contexte social, ainsi que les critiques renouvelées des membres du Parti communiste français sur la religion, contribuent certainement à aiguiser son jugement. Selon lui, si l'institution religieuse ne reconnaît pas, entre autres choses, le caractère intrinsèquement pervers du capitalisme, c'est parce qu'elle se maintient financièrement dans ce système par ses investissements industriels et commerciaux. Non seulement ces considérations matérielles justifient les prises de position idéologiques, mais en plus cette vision du monde sert de rempart contre l'avancée du bolchevisme ; c'est sans doute ce qui explique la collaboration des épiscopats aux politiques des régimes anti-démocratiques d'Italie, d'Allemagne, de France et d'Espagne juste avant et durant la Seconde Guerre mondiale. ${ }^{194}$

Garaudy, en dénonçant par l'intermédiaire d'un tel discours la relation qu'entretient l'Église avec le capitalisme, témoigne de la possibilité chez l'humain de se distancier de ses représentations, d'une façon de voir et d'exprimer les choses. Son

189 Ibid, p. 225.

190 Par exemple : en démocratisant le discours religieux par l'utilisation d'un vocabulaire scripturaire plus accessible à tous, en accordant plus d'attention aux masses exploitées et en faisant l'éloge de la pratique sociale comme accomplissement du dessein de Dieu.

191 Antoine Casanova, Le concile, vingt ans après, Paris, Messidor, 1985, p. 71.

192 Roger Garaudy, Mon tour du siècle en solitaire : Mémoires, Paris, Robert Laffont, 1989, p. 219.

193 Ces propos exprimés par Roger Garaudy sont inspirés d'un bilan dressé par le Père Chenu dans son livre : La doctrine sociale de l'Église comme idéologie, Paris, Éd. du Cerf, 1979. Ces informations proviennent de Roger Garaudy, Biographie du XXe siècle : Le testament philosophique de Roger Garaudy, Paris, Tougui, 1985, pp. 214-215.

194 Ibid., pp. 215-216. 
interprétation des prémisses idéologiques qui justifient ses croyances religieuses, semble de moins en moins correspondre au discours officiel de l'Église, mais beaucoup plus à celui de la communauté épistémique marxiste. Cette dernière s'oppose à toutes formes d'aliénation, qu'elles soient économiques, politiques, religieuses ou autres.

Pourtant, ce qui est remis en cause ne touche pas tant la nature même des valeurs absolues, mais la façon de leur donner une signification au niveau des pratiques sociales. Pour Garaudy, cela est possible par la réalisation d'un projet de vie créatif incitant au dépassement de l'ordre présent et où chaque être humain se rend responsable des autres. Le sens moral de cette responsabilité constitue une sphère de réflexion qui oriente ses décisions. Cette représentation est significative pour lui. Bien qu'il partage des convictions avec d'autres dans l'espoir d'atteindre un idéal, il semble développer, dans son discours sur le catholicisme, une critique de plus en plus pointue des moyens utilisés par cette institution pour accomplir ses fins.

Aussi, lorsque nous analysons les rapports qu'il entretient avec la communauté catholique, nous constatons qu'il est attaché à la frange progressiste du clergé. Ces acteurs sociaux catholiques, sensibles aux misères du peuple, veulent ouvrir l'institution à l'éthique de la discussion, c'est-à-dire démocratiser l'Église et, par ce fait même, bouleverser la fixité des rapports sociaux qu'elle cautionne. Si Garaudy témoigne de son appui à cette cause, la portée de son influence à l'intérieur de cet institution est limitée ; il n'y est pas vraiment reconnu comme un leader d'opinion valable. ${ }^{195}$

\section{3-2.2.4 Du marxisme...}

Retour à la table des matières

En contrepartie, son ascension au sein du Parti communiste lui donne beaucoup plus d'espoir de réaliser son idéal. Néanmoins, même à l'intérieur de ce cadre de négociation des significations, il se retrouve confronté à des divergences d'interprétation importantes concernant la façon d'atteindre les objectifs fixés par les membres du groupe. Trois raisons principales motivent sa dissension : les révélations de Khrouchtchev divulguant l'ampleur de la répression en Union Soviétique sous Staline; l'impérialisme militaire soviétique (plus spécifiquement en Hongrie, en Tchécoslovaquie, et en Afghanistan), mais aussi politique et idéologique (en Chine et

195 Le 5 novembre 1981 (avant sa conversion), Roger Garaudy rencontre le Pape Jean-Paul II à qui il remet deux de ses livres : Karl Marx et L'Islam habite notre avenir. De la lettre de remerciements qu'il recevra par la suite, Garaudy dit à propos du Pape : «[...] manifestement, ni la compréhension de Marx, ni celle de l'Islam, ne sont ses problèmes.» Ibid., p. 233. 
en Yougoslavie) ; et l'intégration en Union Soviétique du modèle occidental de croissance. ${ }^{196}$

Ces critiques méritent d'être expliquées plus amplement car elles révèlent la nature ainsi que l'ampleur des dilemmes moraux qui vont assaillir Garaudy et qui vont le pousser à se retirer de la communauté épistémique communiste, avant de joindre la communauté musulmane.

Il faut souligner d'emblée que Garaudy, même s'il reconnaît par son alliance au Parti communiste français, avoir commis des erreurs, réitère à plusieurs reprises ne pas regretter son adhésion initiale au Parti. Un idéal motivait sa décision : en accord avec la pensée de Marx, libérer l'humain de toutes les formes d'aliénation économiques, sociales, politiques et religieuses. Pour lui, l'atteinte d'un tel objectif ne signifie pas la négation de la foi religieuse, car ce système de croyances peut aussi permettre de saisir les formes aliénées de la religion. Concrètement, cela signifie dans le contexte social de l'avant-guerre, militer contre l'avancée du fascisme hitlérien. C'est en relation à cette quête d'idéal, significative pour lui, que Garaudy s'engage auprès des Communistes.

Les représentations qui y sont associées ainsi que les actions pour y parvenir vont toutefois s'avérer fort différentes pour les dirigeants communistes soviétiques. Contrairement à la religion qui ne propose pas de changer l'ordre social ici-bas parce qu'elle conçoit la plénitude dans l'au-delà, les actions politiques menées par les dirigeants communistes soviétiques entendent remédier à cet état de choses. Leurs décisions s'inspirent de la théorie de l'utopisme qui consiste à «vouloir bâtir une société parfaite par les seuls efforts des hommes, sans aucune référence à Dieu [...]. » 197 Avec l'avènement des États totalitaires, l'utopisme va devenir une doctrine mieux connue sous le nom de scientisme. ${ }^{198}$ Ce schème d'interprétation ne constitue en rien une pratique scientifique. Au contraire, il limite la compréhension des objets de connaissance en ne tenant pas compte du décalage entre les principes et la pratique. Ainsi, selon ce paradigme, chaque objet de connaissance peut être connu par la raison humaine. Pour y parvenir, il suffit d'appliquer le savoir par l'intermédiaire d'une pratique scientifique désignée. La « science » en devenant réponse à tous les problèmes prend valeur de vérité. Il n’y a plus de tolérance pour les hypothèses réfutées.

Sur le plan politique, l'introduction du scientisme va se traduire par l'imposition d'une vision unique des pratiques sociales, ce qui aura pour conséquences de limiter la liberté et l'autonomie des personnes. En ne tenant plus compte de l'opinion des

\footnotetext{
196 Ibid., p. 190.

197 Nous croyons qu'il ne faut pas confondre l'utopisme avec le principe de laïcité. Ce dernier définit la séparation entre le pouvoir de l'État et celui de l'Église. La texte cité provient de Tzvetan Todorov, Mémoire du mal, tentation du bien : Enquête sur le siècle, Paris. Laffont, 2000, pp. 2930 .

198 Ibid., pp. 30-35.
} 
autres, le choix entre le bien et le mal s'atrophie, toute l'importance étant désormais accordée à la réalisation des fins peu importe l'impact des moyens utilisés pour y parvenir. L'idéal réside dans le bien-être commun, plus que dans la réalisation individuelle. Le nous du groupe est privilégié aux dépens du je. ${ }^{199}$ Conformément à cette vision du monde, la réalisation du bonheur de l'humanité tel que proclamé dans l'idéologie communiste doit être envisagée sans aucune tolérance pour ses détracteurs. L'idéologie devient ainsi un prétexte pour justifier le pouvoir de l'État ainsi que la conformité idéologique. Cette forme de totalitarisme, pour s'imposer à tous, en vient à justifier la pratique généralisée de la terreur ainsi que l'usage de la violence. Peu importe l'écart avec la pratique, le discours doit en arriver à ses fins, même si cela signifie une transformation du monde, coûteuse en vies humaines. Ainsi, si Lénine contribue à l'avènement de la république soviétique, Staline qui lui succède de 1924-1953 va, par sa volonté d'instaurer la collectivisation des terres ainsi que l'industrialisation du pays, engendrer des conditions de souffrance inhumaine. Ses interventions vont générer une famine généralisée au sein de la classe paysanne. De plus, un million de personnes vont être fusillées, sans parler des neuf millions de prétendus contestataires qui mourront en détention dans des camps de concentration ou en prison. Ces faits révélés par Khrouchtchev, en 1956, au XXe Congrès du Parti communiste de l'Union soviétique, vont ébranler Garaudy.

Il est profondément affecté par ces dénonciations. Avec le recul du temps, Garaudy, réfléchissant sur lui-même dans sa Biographie, relate les événements qui ont contribués à éveiller chez lui un changement de perception par rapport à l'idéologie communiste soviétique et qui vont le conduire à adopter une attitude beaucoup plus critique à l'égard des directives de l'organisation. Il écrit à ce sujet :

Lorsque je rencontrai personnellement Staline, en 1953, avec un comportement si différent de ce que la propagande ennemie lui attribuait, je me suis sentis conforté dans ma reconnaissance pour ce que nos peuples d'Europe lui devaient, face à Hitler, et dans mon admiration pour les réalisations intérieures, sans imaginer le prix que le peuple soviétique avait payé pour cela $[\ldots]$.

Un seul fait alors m'étonna : dans ses propos, seul le monde occidental semblait poser problème. Les peuples des autres continents apparaissent comme des appendices de la classe ouvrière des pays occidentaux. Même la Chine, pourtant représentée, à ce XIXe Congrès du Parti Communiste de l'Union Soviétique, par son Président Liou Chao Chi. Et lorsqu'Ho Chi Minh, avec lequel nous étions alors en guerre pour perpétuer le système colonialiste, me serra dans ses bras, le premier doute surgit de mes larmes, sur la justesse de la politique communiste que personnifiait Staline.

Il me fallut des années pour comprendre ce qu'était la racine des erreurs et des crimes de Staline. 200

199 Ibid., pp. 36-53.

200 Roger Garaudy, Biographie du XXe siècle : Le testament philosophique de Roger Garaudy, Paris, Tougui, 1985, p. 253 
Au contact des têtes dirigeantes du Parti, Garaudy prend conscience des divergences d'interprétation à propos du socialisme et des possibilités de dérapage quant aux moyens entrepris pour y parvenir. $\mathrm{Si}$, au départ, la révolution politique et économique constitue une remise en question du fondement des relations sociales établies sur la base de rapports de domination et d'aliénation, il va se rendre compte des perversions qu'engendre l'idéal communiste lorsqu'il est poursuivi sans considérations universalistes.

Todorov explique les conséquences de cette situation. ${ }^{201}$ L'interprétation du communisme qui se veut porte étendard de la lutte des classes, crée des divisions entre les humains sur la base de leur identité particulière, notamment celle les associant à leur classe sociale. La lutte à mener devient alors un prétexte pour justifier des représailles envers ceux et celles qui y contreviennent ; les autorités politiques font disparaître la monarchie, mais plus pernicieusement elles s'opposent farouchement à toute forme d'opposition idéologique perçue comme telle. Des dirigeants comme Lénine ou, pire encore, comme Staline ne cherchent pas à diriger dans le compromis, mais veulent imposer leur vérité à tous. Ainsi, si initialement, ils limitent leurs interventions à l'intérieur des frontières nationales, rapidement les intérêts de la Russie vont devenir indissociables de la révolution mondiale, d'où cette volonté soviétique d'expansion et d'hégémonie. L'internationalisme soviétique ne constituera donc pas un mouvement de solidarité envers le genre humain, mais une politique ayant pour objectif la défense de l'intérêt national d'un pays à l'étranger. ${ }^{202}$

Staline, qui aurait pu pallier cette disparité entre théorie et pratique, va plutôt chercher à la camoufler. En fait, l'idéologie qui se portait à la défense des intérêts collectifs cède le pas insidieusement aux ambitions personnelles, notamment au désir du pouvoir. En voulant offrir un mode de vie où les interactions sociales s'exécutent selon des considérations idéologiques plus humaines, le socialisme est devenu non pas une alternative au mode de vie capitaliste, mais une forme de totalitarisme mis au service de la défense des intérêts des dirigeants. ${ }^{203}$ La population muselée par un régime de terreur, l'idéologie collective va être perçue progressivement comme une vision du monde incohérente.

Envoyé à Moscou comme correspondant pour l'Humanité (l'organe du Parti communiste français) après la mort de Staline, Garaudy en atteste. Il note le contraste entre le discours et les faits, entre la diffusion généralisée de la propagande apologétique et la possibilité pour la nomenklatura, 204 d'accéder à certains privilèges matériels. De plus, pendant ce séjour d'un an, personne ne l'invite à part trois personnes

201 Tzvetan Todorov, Mémoire du mal, tentation du bien : Enquête sur le siècle, Paris. Laffont, 2000, pp. 17-55.

202 Tzvetan Todorov, Mémoire du mal, tentation du bien : Enquête sur le siècle, Paris. Laffont, 2000 , p. 47.

203 Ibid., p. 53.

204 En U.R.S.S. et dans les pays de l'Est, la nomenklatura, consiste en une classe de privilégiés qui bénéficie de traitements de faveur. 
qui, par leur statut particulier, se situent en marge du Parti et de la société soviétique. Il constate ainsi qu'un climat de suspicion généralisée contribue à entretenir l'expression du conformisme. Il saisit alors l'écart entre sa conception de la pensée de Marx et ce qui se passe en pratique.

À partir de 1962, il devient ainsi l'un des premiers intellectuels à s'investir dans la recherche des erreurs philosophiques de Staline et de leurs conséquences. ${ }^{205}$ Il publie alors, dans les Cahiers du communisme de juillet-août 1962, un rapport où il insiste sur l'importance de la subjectivité dans la connaissance et l'action ainsi que sur la nécessité pour l'idéologie marxiste de se distancier des formes d'interprétation qui la réduisent au positivisme. Sa critique ne consiste pas en une révision du marxisme, mais cherche plutôt à restituer la pensée de Marx. «Et d'en finir avec la pratique dogmatique, dans l'histoire, dans la science, dans la critique littéraire, l'argument d'autorité, la référence aux livres sacrés qui ferme la bouche et rend la discussion impossible. » 206

Et c'est dans cette optique qu'il s'attaque publiquement en 1964 dans les Informations catholiques internationales à la position d'Illytchev, l'un des dirigeants du Parti Communiste de l'Union Soviétique, qui affirme que l'instauration du marxisme ne peut se réaliser tant que la religion perdurera. À cela Garaudy va rétorquer que la foi ne consiste pas en une idéologie, mais en une manière d'être. Les actions des humains ne sont pas uniquement dictées par leurs déterminismes. Lorsqu'ils entreprennent une révolution, les humains témoignent aussi de leurs facultés télésitiques, ou selon l'expression de Garaudy de leur «transcendance ». Ce point de vue, va lui attirer des reproches tant de la part des membres de son Parti que de l'épiscopat français qui ne cautionne pas ses idées en matière de religion; si pour les uns la religion est source d'aliénation, pour les autres elle ne peut inspirer des fins révolutionnaires.

Ces oppositions n'empêchent pourtant pas Garaudy de poursuivre ses interventions destinées à clarifier la pensée de Marx. Il publie ainsi en 1966, Marxisme du XXe siècle, avec lequel il tente de rallier les militants de son Parti ainsi que les membres du Bureau politique auquel il appartient. S'il souhaite réaligner le discours du mouvement, le moment semble plus propice à la confrontation, qu'à l'union des esprits ; l'Union Soviétique ainsi que son Parti condamnent l'interprétation chinoise du marxisme. Pour Garaudy, la faiblesse de l'argumentation de ces organisations politiques est telle qu'elle suscite chez lui une désillusion profonde ; il sombre dans la dépression pendant plusieurs mois. Les différences d'interprétation à propos de la vision du monde marxiste suscitent d'énormes tensions, en lui.

Pour Garaudy, le marxisme ne consiste pas en une formule dogmatique qui peut être appliquée peu importe la situation historique et contextuelle. Si Marx a su entre-

205 Roger Garaudy, Mon tour du siècle en solitaire : Mémoires, Paris, Robert Laffont, 1989, p. 166.

206 Roger Garaudy, Biographie du XXe siècle : Le testament philosophique de Roger Garaudy, Paris, Tougui, 1985, p. 256. 
voir les conditions sociales qui allaient permettre l'essor de la bourgeoisie et le renversement par cette classe sociale de la monarchie pour accéder au pouvoir, ce processus ne peut que différer selon l'état de développement des sociétés. Ainsi, selon Garaudy, l'Union Soviétique ne peut emprunter un cheminement conforme aux prévisions de Marx puisqu'à la veille de la Révolution d'octobre il n'existe pas de classe ouvrière suffisamment organisée pour revendiquer le pouvoir à elle seule. Aussi, l'objectif, pour Lénine, va-t-il consister, au départ, à prendre le pouvoir pour ensuite réunir les conditions économiques qui vont favoriser le développement de la classe ouvrière. Mao, à son tour, effectue aussi une adaptation des théories de Marx selon les circonstances. Mais il semble que les dirigeants soviétiques, aux prises avec des ambitions hégémoniques, maintiennent leur dogmatisme ; tous les Partis, à l'extérieur de l'Union Soviétique, doivent suivre les directives du Parti «central ». Garaudy n'endosse pas cette position. Il est en faveur de la spécificité de l'expérience socialiste dans chaque pays, notamment en France surtout durant les événements de mai 1968.

Mais, la direction du Parti communiste français alignée sur les politiques de Moscou n'entend pas reconnaître cette distinction et, par conséquent, l'issue des révoltes qui paralysent la société française. Elle s'aligne plutôt sur les positions de Marchais, un membre influent du Bureau politique qui ne souhaite pas renouveler le discours du Parti en l'adaptant aux exigences du mouvement de contestation. L'organisation privilégie le retour à l'ordre afin de ne pas compromettre ses chances de remporter les élections qui s'annoncent sous peu. Mais après l'occupation de la Tchécoslovaquie par l'Union Soviétique en août 1968, la poursuite opiniâtre d'une telle orientation politique devient de plus en plus inappropriée, selon Garaudy. L'écart de perceptions quant à l'analyse de la situation devient une source de conflits irrémédiables entre lui et les autres membres du Parti. En 1970, il quittera cette organisation dont il aura été solidaire pendant 37 ans. Il écrit à propos de sa dernière intervention au XIXe congrès du Parti communiste français :

Mes derniers mots sont suivis d'un silence horrible. [...] J'ai l'impression de tomber dans un puits en allant me rasseoir au milieu de ces deux mille camarades, dont la plupart étaient hier encore mes amis.

Pas un, aujourd'hui, même parmi ceux qui partagent certaines de mes positions, n'a osé s'exprimer à mes côtés. La séance est levée, l'on s'écarte de moi comme devant un lépreux. ${ }^{207}$

Comment se fait-il que Garaudy en arrive à l'exclusion? La plupart des personnes auraient peut-être exprimé leurs doutes. Mais peu se seraient avancés jusqu'à compromettre leur carrière ainsi que le soutien des membres de leur réseau de relations après tant de temps et d'expériences vécus ensemble. Dans la lutte pour accomplir son idéal pourquoi continue-t-il, sans relâche, à susciter l'opposition au sein des

207 Roger Garaudy, Mon tour du siècle en solitaire : Mémoires, Paris, Robert Laffont, 1989, pp. 251-252. 
communautés catholiques et marxistes, puis éventuellement auprès de la communauté juive (surtout que cette démarche va lui coûter le droit de publication en France) ? 208

Un des facteurs qui peut sans doute expliquer un tel comportement touche à cette double appartenance identitaire que Garaudy a entretenu toute sa vie. Comme nous l'avons dit précédemment, dès son jeune âge, il est sensibilisé et interpellé par un discours familial qui lui présente deux visions de l'identité religieuse ; rejetant l'idée du néant, il décide, au fil de ses rencontres et de sa formation académique, de croire en Dieu mais aussi en l'humain. Si, au départ, il semble beaucoup plus proche d'un réseau de relations qui partagent des croyances chrétiennes, la guerre qui se profile l'incite à s'investir dans l'action auprès des communistes. Garaudy adhère ainsi à deux communautés de pensée distinctes, à l'intérieur desquelles l'interprétation de la « réalité » varie, mais où l'on retrouve aussi des discours qui, bien qu'ils s'expriment en des termes différents, font référence à des considérations éthiques similaires. C'est dans cet espace de propos communs que Garaudy construit son projet de vie. Si cette position lui donne l'avantage de comprendre les particularités des uns par rapport aux autres ainsi que leurs similarités, cette compréhension de l'autre en fait un traducteur non seulement de mots et d'idées, mais aussi de schèmes d'appréhension de la « réalité ».

Conséquemment, lorsque ces deux communautés de pensée s'affrontent dans l'arène publique, il ne décode pas ces échanges de la même façon qu'une personne qui ne conçoit qu'une de ses deux visions du monde. Le traducteur peut vouloir faciliter la communication entre parties opposées ou encore utiliser les informations relatives à un groupe aux dépens de l'autre. Dans le cas de Garaudy, cette situation est difficile à établir parce que ses préoccupations au sujet des communautés chrétiennes et marxistes semblent se modifier dans le temps. Par contre, ce qui semble beaucoup plus évident à la lecture de son Testament et de sa Biographie, ce sont les raisons qui motivent son adhésion à ces deux écoles de pensée, et surtout la nature de ses erreurs personnelles qu'il associe aux moyens utilisés par ces organisations pour atteindre leurs fins. À cet égard, nous remarquons dans son discours que c'est son adhésion au marxisme qui lui apparaît le plus lourd de conséquences.

208 En 1995, Garaudy publie Les mythes fondateurs de l'État d'Israël. Dans cet ouvrage, il remet en question des faits que certains qualifient d'indiscutables à propos de l'Holocauste juif : par exemple, l'existence de chambres à gaz dans les camps nazis. Aussi est-il accusé, conformément à la loi Gayssot (adoptée en 1990), qui sanctionne les propos révisionnistes cautionnant l'antijudaïsme. Garaudy est condamné a versé une amende considérable (entre 120000 et 160000 francs). Cela a aussi pour conséquence qu'il ne peut plus, désormais, publier en France. Pour sa défense, Garaudy dira qu'il ne nie pas les crimes Nazis, ni les persécutions que les Juifs ont dues subir durant la Seconde Guerre mondiale. Il dit vouloir dénoncer la propagande sioniste qui est utilisée comme base de revendications pour justifier la création et le maintien de l'État d'Israël. L'abbé Pierre le sou-tiendra durant cette tourmente. Pour notre part, nous commentons ces événements pour démontrer d'autres motifs d'adhésion, pour Roger Garaudy, à la communauté musulmane et non pour prendre position dans ce débat. Rappelons que cette affaire est postérieure à sa conversion religieuse (1982). http://www.republique-des-lettres.com, http://www.adl.org, http://www.codoh.com. 
Au départ, la réalisation de l'idéal collectif de cette communauté ne semble pas lui poser un problème éthique grave. Même lorsqu'il pourrait enregistrer des signes pouvant semer le doute dans son esprit, comme par le massacre de Katyn, il ne les considère pas vraiment. ${ }^{209}$ Mais, leur accumulation ainsi que la constatation que le discours officiel soviétique en soit venu à déclarer ouvertement son mépris religieux, ont dû contribuer à éveiller sa sensibilité critique de sorte que l'illusion de l'idéal s'est effondrée pour faire place à plus de discernement critique à l'égard du discours de son propre groupe. Et c'est ultimement l'ensemble de ces incongruités interprétatives qui vont soulever les conflits incessants entre lui et les autres parce qu'ils sont dépourvus de cette double appartenance et de cette possibilité de distanciation critique. Cette situation va contribuer à son isolement et son exclusion éventuelle de la communauté épistémique marxiste. $\mathrm{Si}$, au départ, on le considère, au sein des organisations qu'il fréquente, comme un émissaire négociant les discours des deux communautés de pensée auquel il adhère, à la fin, les communistes fidèles à la ligne de pensée soviétique en viennent à le percevoir comme un traître.

Ainsi remarquons-nous dans l'expérience de Garaudy, des similitudes avec l'histoire de la Malinche. Il doit constamment justifier son allégeance auprès des uns et des autres. De plus, dans chacune des organisations respectives auxquelles il adhère, il s'identifie aux clans dissidents. Comme il connaît bien les présupposés des uns et des autres, il réalise, comme La Malinche, des opérations de réception active. Il se distancie inexorablement du discours idéologique officiel défendu par les membres ainsi que la direction de ces organisations. Il réitère, inlassablement, sa vision du monde à l'intérieur de chacune de ces organisations de trois façons : en faisant valoir son discours personnel qu'il remet à jour régulièrement; en s'opposant à certains arguments qui ne valorisent pas les mêmes présupposés éthiques; en désarticulant les propos des uns, par rapport à ceux des autres, de façon à questionner les variations d'interprétation en fonction des changements contextuels.

Pour Garaudy, chacune de ses appartenances identitaires (catholique et marxiste) se complète et revêt une signification cohérente. Dans un monde idéal, il souhaiterait peut-être que l'Église devienne révolutionnaire et le Parti communiste plus respectueux des normes religieuses. Cette quête d'un espoir chez l'autre l'empêche de se replier sur lui-même, ou encore de se laisser totalement séduire par lui. L'ouverture d'esprit ainsi que la tolérance à l'égard de l'autre qu'il cultive lui permettent d'entretenir une relation dialectique avec le discours de communautés épistémiques en désaccord sur différentes interprétations d'idées. Ce processus, tout en facilitant la mise à jour de sa pensée, l'aide à construire son projet de vie selon ses croyances morales. Cette façon de communiquer est bien ancrée chez lui et fait partie du rôle qu'il

209 On découvrit en 1943 aux abords de la ville de Katyn en Russie des fosses communes dans lesquelles étaient enterrés plus de 4500 officiers polonais. Tant les Allemands que les Soviétiques nièrent leur responsabilité dans cette affaire. En 1953, une commission d'enquête américaine conclut que ce massacre avait été perpétré par la police soviétique. Petit Robert 2, Dictionnaire universel des noms propres. 
s'est donné en tant qu'intellectuel. Mais, à partir du moment où cette vision du monde s'effondre, on peut se demander ce qui le pousse à vouloir se joindre à une autre communauté d'interprétation.

\section{3-3 Résolution des conflits d'interprétation par la conversion}

$\underline{\text { Retour à la table des matières }}$

Comme nous l'avons expliqué précédemment, Garaudy croit en une forme d'immanence où l'humain ne se suffit pas à lui-même, mais respecte des normes morales religieuses sans s'aliéner. Pourquoi défend-t-il si ardemment cette position en apparence ambivalente? Sans doute, sa conviction que l'humain peut faire preuve de grandes vertus morales l'incite à s'opposer à tout système de pensée qui le réduit à des déterminismes de classe, de race, de religion, etc. Nous croyons que si cette croyance est évoquée avec autant de conviction, c'est qu'elle est associée, pour Garaudy, à un événement marquant de sa vie. Nous allons raconter cette expérience car elle trace aussi le cheminement de la construction de sa conversion à l'islam. ${ }^{210}$

Au début de la Deuxième Guerre mondiale, Garaudy est considéré par les autorités du régime de Vichy comme un Propagandiste Révolutionnaire. On l'affecte donc à la «Septième DINA » (Division d'Infanterie Nord-Africaine) en Algérie, aux confins du Sahara. Au côté des Arabes marocains, algériens et tunisiens, vont se trouver à combattre des résistants au fascisme que l'on a envoyés combattre aux points les plus meurtriers. Le 4 mars 1941, au moment où un convoi de volontaires étrangers vient se joindre à eux, Garaudy, ainsi que tous les réfractaires de son camp, contreviennent à l'ordre du commandant français de s'enfermer dans leurs marabouts. Ils entonnent Au-devant de la vie... Le commandant, incapable de censurer cette impulsion contestataire donne l'ordre à la garde de tirer. Garaudy, l'espace d'un instant, croit qu'il va mourir. L'attente se fond en silence. La notion de vivre l'instant présent prend alors une autre signification ; comment, remettre à plus tard, désormais, ce qui est possible d'être accompli maintenant. Il n'a que vingt-huit ans. Mais ils ne tireront pas. Ces hommes, des « ibadites », font partie d'une secte musulmane. Leurs croyances religieuses leur ont valu, il y a mille ans, d'être poursuivis jusqu'au Sahara. C'est en réponse à l'appel de Dieu qu'ils vivent, depuis, dans cet environnement hostile. Garaudy dit de ces derniers :

210 Il en parle dans son Testament, pages 277-280, et dans sa Biographie, pages 64-70 et 340. 
Ces inconditionnels de Dieu nous ont fait vivre : il est contraire à l'honneur de guerriers musulmans du Sud qu'un homme armé tire sur un homme désarmé. Ils avaient, avant nous, l'expérience de la transcendance vécue. ${ }^{211}$

On peut penser qu'à partir de cet événement, l'appréciation de l'autre n'est plus perçue de la même manière. Bien que l'Arabe ou le Français appartiennent à des cultures différenciées où l'activité créatrice et spirituelle humaine s'expriment selon des considérations différentes, on remarque que des valeurs immuables voire universelles transcendent aussi ces appartenances particulières. Pour Garaudy, « tout prend un sens à partir de cette fin »; ${ }^{212}$ la fin possible de son être, la fin de son attachement identitaire spécifique, et la réalisation d'un projet de vie universaliste en tant que fin. Les fins de l'être et de l'attachement identitaire sont saisies en termes de finitude, c'est-à-dire qu'elles laissent entrevoir la fin d'un terme. Alors que la fin du projet de vie universaliste devient une finalité, un but vers lequel tendre. Bien que ces prises de conscience distinctes semblent être intégrées par moments séparés dans le temps, elles sont complémentaires. Le détachement identitaire et la recherche d'un projet de vie universel donne un sens nouveau à sa vie. Mais à son tour, ce sens est revisité ; Garaudy reconnaît sa dette envers les Ibadites ce qui le prédispose à la conversion.

C'est ce que nous constatons à la suite de la lecture de son Testament et de sa Biographie. L'interprétation de l'expérience de sa mort avortée et le contexte où elle se situe vont, par la suite, affecter sa vision du monde. C'est ainsi qu'après sa libération en 1943, il en vient à entreprendre, comme assistant à la direction du Parti communiste en Algérie, une série de conférences par l'intermédiaire desquelles il souhaite établir des ponts entre la culture française et la culture arabo-islamique afin de mieux combattre le racisme nazi. Si cette idée l'amène à développer un discours qui met en valeur la contribution historique et culturelle de la civilisation arabo-islamique, elle l'incite aussi à critiquer sévèrement les colons français d'Algérie qui ont collaboré avec l'envahisseur allemand. Tout comme la Malinche qui rejette son statut d'esclave et la culture qui le lui a imposée, Garaudy, en refusant de cautionner la communauté française qui soutient le régime de Vichy, en vient à remettre en perspective son attachement à son identité culturelle nationale. Cette prise de conscience facilite la reconnaissance de l'universel en l'autre et du particularisme en soi.

En s'identifiant au mouvement de Résistance, les critères d'identification à la nation s'estompent pour faire place à une forme de solidarité qui dépasse les frontières. Tous ceux et celles qui s'investissent pour défendre le flambeau de la liberté s'unissent aux dépens de leurs allégeances particulières. Cette situation favorise la rencontre de tout un réseau de relations qui autrement ne se seraient peut-être pas parlées.

La rencontre de Garaudy avec le cheikh Ibrahimi se situe probablement dans un tel cadre. Le cheikh, symbole de la Résistance et représentant reconnu du soufisme,

211 Roger Garaudy, Mon tour du siècle en solitaire : Mémoires, Paris, Robert Laffont, 1989, p. 66.

212 Ibid., p. 65. 
lui fait découvrir une forme d'expression spirituelle qui ne valorise pas tant le détachement du monde matériel, que l'acte réflexif qui médite sur l'expression de la foi dans l'action. Cette prise de conscience des possibilités de construction de « réalité » à partir des représentations religieuses, devient une problématique importante pour Garaudy. Si, à partir de cette révélation, il découvre dans l'islam une variété de doctrines idéologiques pouvant intégrer des provenances politiques autant de gauche que de droite, la lutte contre le nazisme lui ouvre des portes d'accès à des réseaux de résistance à l'intérieur de l'univers musulman qui, dans d'autres circonstances, auraient peut-être été inaccessibles. ${ }^{213}$ Le contexte de la guerre, en stimulant des élans de solidarité au-delà des barrières identitaires favorise la rencontre de leaders d'opinion appartenant à des mondes différents. ${ }^{214}$ Pour Garaudy, le développement de ces relations en marge de son identité primaire va se constituer en un réseau de contacts secondaires qu'il va solliciter personnellement, après son exclusion du Parti communiste, afin d'atténuer sa crise interprétative. Ces contacts lui seront également utiles, plus tard, afin de contrevenir l'interdit de publication qui va restreindre sa liberté d'expression en France. La conversion religieuse de Garaudy, en 1982, lui offre la possibilité de publier à nouveau ses écrits, mais cette fois, à l'intérieur d'une nouvelle communauté épistémique. Garaudy dit d'ailleurs sur ce point «prendre conscience qu'une «conversion » n'est pas nécessairement un changement de la foi, mais de la culture dans laquelle elle s'exprime. » ${ }^{215}$ La divulgation de sa conversion va d'ailleurs se répandre rapidement dans les communautés musulmanes d'Occident, à la suite de quoi, de nombreuses invitations vont lui être faites de nouveaux réseaux de reconnaissance.

La conversion religieuse nécessite, toutefois, que l'apprentissage des conventions qui régissent les comportements sociaux de la nouvelle culture adoptée soit fait sensiblement de la même façon que lors de la socialisation primaire. Les personnes investies dans un processus de socialisation secondaire sont également affectées dans leur identité par la coloration affective des rapports humains établis auprès des nouveaux agents de socialisation. La conversion peut paraître comme un moyen d'autant plus attirant pour régler un problème d'interprétation auprès d'un nouveau groupe de relations, que dans certaines communautés religieuses, comme c'est le cas chez les musulmans, le rôle et le statut de l'intellectuel sont reconnus et appréciés socialement. D'ailleurs, dans un hadith du prophète Mahomet, c'est-à-dire l'une de ses paroles souvent récitées, il est dit : «l'encre des savants est pour moi plus précieuse du (sic) sang de martyrs. ${ }^{216}$ On peut donc penser que si la conscience d'anomalies constitue une

213 L'islam offre, non seulement l'accessibilité à des communautés d'interprétation variées, mais en plus, la possibilité de rencontrer et de vivre une relation privilégiée avec des maîtres, sans nécessairement se couper du reste du monde. Stefano Allievi, Les convertis à l'islam : Les nouveaux musulmans d'Europe, Paris, L'Harmattan, 1998, pp. 130-142.

214 Entre autres personnalités du monde arabo-islamique, Garaudy va rencontrer l'impératrice d'Iran, Farah Diba, ainsi que Nasser et Kadhafi.

215 Roger Garaudy, Mon tour du siècle en solitaire : Mémoires, Paris, Robert Laffont, 1989, p. 228.

216 Stefano Allievi, Les convertis à l'islam: Les nouveaux musulmans d'Europe, Paris, L'Harmattan, 1998, p. 126. 
condition préalable à la recherche d'une nouvelle vision du monde, la reconnaissance d'un statut social privilégié à l'intérieur d'une nouvelle communauté de pensée contribue ultimement à séduire les nouveaux fidèles.

De plus, l'adhésion à l'islam n'entraîne pas pour le Juif ou le Chrétien de devoir renoncer à ces croyances initiales ; ces deux religions du Livre sont reconnues dans le Coran. ${ }^{217}$ Le fait de ne pas avoir à abandonner le schème de ses représentations primaires semble conforter Garaudy dans sa démarche de conversion. De la doctrine de l'islam, il dit n'avoir su que très peu de chose mis à part les connaissances acquises par ses lectures ainsi que l'expérience de la transcendance vécue lors de la tentative de fusillade de Djelfa. ${ }^{218}$ Ce qui nous porte à croire que cette conversion, en tant que solution interprétative, s'inscrit dans la continuité d'une démarche rationnelle, voire intellectuelle de recherche de significations, mais comporte aussi un volet pratique associé à un réseau de relations. 219

$\mathrm{Si}$, ce réseau lors de son premier séjour en Algérie ne semble pas vraiment constitué, l'expression de la foi musulmane dans la pratique ne le laisse pas indifférent. L'événement de Djelfa a laissé un souvenir impérissable dans sa mémoire. On sent de la reconnaissance, dans son témoignage, sur cet événement. Cette situation contribue à nourrir une ouverture d'esprit par rapport à l'Islam ${ }^{220}$ qui rend accessible l'exploration des significations qui y sont associées.

Toutefois, tant que son interprétation des idéologies chrétiennes et marxistes ne posera pas de problèmes moraux sur le sens des actions à entreprendre, Garaudy continuera à se sentir solidaire des autres membres des groupes auxquels il appartient, et la considération de l'Islam comme alternative religieuse ne sera pas envisagée. Néanmoins, les conflits d'interprétation liés à l'éthique vont changer la dynamique relationnelle dans laquelle il évolue, au point où, il ne reconnaîtra plus l'adhésion des autres à ses valeurs. L'accord tacite qui les unissait sera rompu. Et c'est plutôt au sein de l'Islam, de son groupe de relations secondaires, que viendra la possibilité de réitérer à nouveau, et conformément à ses valeurs, sa vision du monde selon les modalités d'expression qui lui conviennent. Garaudy pourra en tant qu'intellectuel continuer de faire réfléchir et de faire connaître sa quête de significations.

Et, comme dans bien des cas de conversion, ce n'est bien souvent qu'après le rituel d'entrée dans la nouvelle organisation que la participation active à la nouvelle communauté de pensée deviendra incontournable. Pour bien des convertis, comme il semble également que ce soit le cas pour Garaudy, le besoin de se socialiser afin d'in-

\footnotetext{
217 Ibid., p. 44.

218 Roger Garaudy, Mon tour du siècle en solitaire : Mémoires, Paris, Robert Laffont, 1989, p. 340.

219 Ces propos nous sont inspirés par Stefano Allievi, Les convertis à l'islam, les nouveaux musulmans d'Europe, Paris, L'Harmattan, 1998, p. 94-95.

220 Le terme islam correspond à la religion prêché par Mahomet. L'Islam (avec I majuscule), représente l'ensemble des peuples qui professe cette religion. Mais, selon nous, l'Islam est aussi associée aux réseaux de relations que le converti intègre.
} 
térioriser sur le plan cognitif le nouveau paradigme se fait sentir après l'acte de conversion. Cet apprentissage qui peut comporter de multiples variations en apparence, est également très subtil. Il ne consiste pas simplement dans l'acquisition d'un nouveau savoir religieux. Il réside plutôt dans la faculté télésitique d'interpréter ses valeurs selon un nouveau schème d'interprétation et, peut-être même, d'acquérir de nouvelles représentations afin que soient résolus les problèmes antérieurs associés à certaines significations. Ensuite, la difficulté consiste à transposer cet ensemble de connaissances nouvellement acquises dans un mode de comportement acceptable, répondant aux attentes et aux normes du groupe dans lequel le converti cherche à se fondre. En fin de compte, le besoin initial d'être cohérent avec ses croyances spirituelles, peut conduire à vouloir collaborer, avec d'autres, à l'établissement d'un sens collectif. 


\section{Conclusion}

Retour à la table des matières

Dans l'introduction de ce mémoire, nous disions vouloir expliquer le phénomène de conversion religieuse, non pas comme une expérience que les personnes subissent au contact du discours de personnalités charismatiques, mais plutôt démontrer la construction d'un tel processus selon leur capacité d'y donner une signification cohérente. Afin de parvenir à notre but, nous avons fait valoir, à maintes reprises, que les êtres humains, contrairement aux animaux, peuvent s'émanciper de leur conditionnement socialisé pour interpréter en fonction de nouvelles considérations identitaires leur « réalité ». Cette faculté, que Lee Thayer évoque par le terme «télésitisme», permet aussi à l'humain de décoder le monde en prenant en compte d'autres points de vue.

Toutefois, dans l'habitude, l'humain a tendance à se complaire dans la reproduction de l'identique et à vouloir confirmer son schème d'appréhension de la vie en faisant valoir la justesse de son interprétation auprès des autres. Pour y parvenir, il manifeste aussi ses désaccords par rapport à la position des autres. Mais il peut également faire preuve d'empathie. En d'autres termes, il lui est possible d'arriver à prendre conscience, au contact des autres, de leur conception du monde. Ainsi apparaît la relativité des Weltanschauugen, c'est-à-dire la variabilité des façons d'appréhender culturellement «les réalités ». C'est sur ce principe que se fonde la pratique de la réception active. Ce type de décodage n'est pas exceptionnel. Tous s'y emploient. Mais il est possible de s'y investir dans des registres d'intensité variés. Certaines personnes, à la rencontre de l'autre en eux et à l'extérieur d'elles, arrivent à se distancier d'elles-mêmes au point d'entrevoir la relativité de leur schème d'appréhension. 
La pratique de la réception active fait appel à l'ensemble des facultés cognitives ; que l'on parle de mémoire, d'imagination, de perception, d'intuition, ou de jugement, toutes peuvent contribuer à raviver les expériences déterminantes de notre vie, selon les situations, et stimuler la pratique réflexive. La réflexivité peut permettre de les réactualiser dans de nouveaux registres contextuels ou de les adapter aux variations du temps dans l'espace.

Ces possibilités diverses d'affirmation de soi ouvrent la voie de l'être multiple. La tradition ne pouvant plus toujours conduire la réflexion face à l'ensemble des questions soulevées dans la modernité, l'humain se voit contraint d'assumer sa liberté en tentant d'y répondre. Cette situation l'amène à s'investir, selon son statut social et ses intérêts, dans des rôles différenciés en tant que parent, professionnel, membre d'une association, qu'il doit concilier afin de donner une signification à son existence conformément à sa vision du monde. Or, si les êtres humains, tendent à maintenir le discours relatif à leurs identifications identitaires, ils peuvent être ébranlés par de nouvelles interprétations du monde, ou encore par l'impossibilité d'arriver à rendre compte selon des présupposés connus de la nouveauté de changements contextuels. Cette prise en compte, lorsqu'elle a lieu, peut provoquer des situations de conflits au sein des groupes d'interprétation, à propos des valeurs fondamentales qui orientent la perception et l'énonciation du discours. Ils risquent alors de ne plus s'identifier aux membres des communautés avec lesquels ils étaient solidaires jusqu'alors.

Si l'être humain issu de la modernité, peut exercer la pratique réflexive avec plus de latitude, il peut aussi renouveler le sens de ses relations avec les autres lorsqu'il ne s'identifie plus à l'interprétation collective. Il peut ainsi décider de se joindre à un nouveau groupe de personnes qui partage une réalité communicationnelle plus conforme à son interprétation des changements.

L'ensemble de ce processus ne survient donc pas sans l'implication active du converti à vouloir remédier à ce qui l'oppose aux autres. Le converti ne nous apparaît donc pas comme un être impuissant, soumis à la volonté d'un manipulateur, mais plutôt comme un acteur social qui, en possession d'une carte radar personnalisée, circule dans des univers de significations variées qu'il décode activement selon sa reconnaissance des présupposés et valeurs morales qui orientent son appréhension de la vie. On comprendra que l'on ne parle pas ici de contacts superficiels, mais bien de relations suivies et approfondies qui se développent avec le consentement des parties en présence.

Et, tout comme lors de la socialisation primaire à l'intérieur des groupes d'appartenance, la prise en compte d'une nouvelle matrice de références pour interpréter le monde se constituera par les pressions coerséductives répétitives qu'exerceront les membres de la nouvelle communauté. Ceci, afin de rendre confor- 
mes l'interprétation et les comportements des nouveaux adhérents. La conformité des significations facilite les échanges et les interactions collectives. Les réalités communicationnelles partagées créent l'illusion de l'appréciation d'une vérité à propos de la « réalité ». C'est sans doute ce qui explique pourquoi les personnes qui se désolidarisent d'un groupe, apparaissent aux yeux de celles qui continuent à revendiquer la même identité comme des traîtres, parce qu'elles ne cautionnent plus les mêmes valeurs de vérité. Inversement, on pourrait aussi les considérer comme des traducteurs d'univers communicationnels distincts.

L'expérience de la conversion religieuse procède donc de cette possibilité de s'immiscer dans des univers communicationnels étrangers pour s'approprier de nouvelles significations. C'est ce dont nous avons essayé de rendre compte dans notre analyse. Pour y parvenir, nous avons pris en considération comment le converti en arrive à rendre son changement d'adhésion significatif, pour lui, et en relation aux autres. Comme nous l'avons expliqué précédemment, les diverses approches qui cherchent à décortiquer le phénomène plutôt que d'essayer de reconstituer sa construction, nous apparaissent insatisfaisantes. C'est ce à quoi nous avons tenté de remédier par ce travail.

Afin de réaliser notre objectif, nous avons exposé dans notre problématique, la façon dont l'acteur social s'interprète en relation avec les autres et les produits de l'activité humaine, tout en considérant la façon dont il assimile les changements contextuels. Nous avons également expliqué comment l'appréhension du monde est tributaire des attachements identitaires des personnes, c'est-à-dire du schème d'appréciation qu'elles ont développé initialement lors de leur socialisation primaire et par lequel elles confèrent au monde la signification qu'il a pour elles. Évidemment ce processus de subjectivation de la « réalité », acquis dans la joie, la peine et l'effort auprès des autorités compétentes, suppose que les individus vont vouloir se sécuriser en préservant leur prise en compte du monde. L'expérience de la conversion nous démontre, toutefois, qu'il est possible pour l'humain d'user de sa liberté afin de rompre, en partie, ses attaches identitaires et les représentations qui y sont associées. On comprendra que renoncer complètement à un mode d'assertion sur le monde semble impossible, ou plutôt proche de la folie, puisque cela supposerait la déconstruction de toutes les représentations qui donne l'illusion d'une emprise sur la «réalité ». L'humain ne peut faire totalement abstraction de la plaque photographique initiale qui a imprimé en lui certains conditionnements. Si cela n'est pas irrévocable en soi, cette conception du monde module le balayage radar lors de l'acte perceptif ainsi que lors de l'interprétation.

Toutefois, en gagnant de l'autonomie l'acteur social pourra effectuer des choix et se démarquer des conventions érigées par les membres des communautés d'appartenance qui ont participé à sa socialisation. Mais, pour arriver à prendre en compte le discours d'une communauté épistémique perçue comme marginale par les membres de la communauté d'appartenance, l'acteur social a besoin de réfléchir sur 
son expérience de vie, afin de concevoir les raisons de son désistement ainsi que son attirance envers les membres d'un nouveau groupe.

Les recherches de « l'École de Columbia » ont démontré que l'adhésion à un discours «étranger» est fonction de la qualité de son contenu, des valeurs que défend son porteur ainsi que le réseau, restreint et élargi, de personnes qui semblent associées à ce leader d'opinion. Considérant toutes ces données, nous nous demandions, principalement en début de travail, comment s'effectuait cette transition identitaire, d'un groupe à un autre, sur le plan religieux. De même nous nous questionnions, mais de façon beaucoup plus secondaire, sur la façon dont un leader d'opinion en vient à effectuer une telle transition. Plus précisément, comment, à la suite d'un conflit d'interprétation, il en vient à délaisser les communautés épistémiques auxquelles il adhère pour éventuellement partager avec un autre groupe une vision nouvelle du monde, et quels sont les avantages d'une telle pratique.

Pour répondre à nos interrogations, nous avons, dans un premier temps, essayé de saisir le processus par lequel l'humain attribue des significations à son existence en tenant compte de ses déterminismes et sa capacité d'agir librement. Nous nous sommes alors demandée comment il élabore la construction de sa « réalité » et comment il peut en arriver à se concevoir autrement par sa communication intrapersonnelle, ses échanges avec les autres et son interprétation du contexte. Dans un deuxième temps, nous avons voulu vérifier nos propos en nous concentrant sur l'analyse d'une seule étude de cas, afin de discerner, avec minutie, dans l'ensemble du parcours de vie d'une personne, en l'occurrence Roger Garaudy, la construction d'une expérience de conversion religieuse.

En procédant de la sorte, nous voulions, faire comprendre les limites de l'approche scientifique ainsi que celles de toutes les approches unidimensionnelles qui restreignent la compréhension du phénomène de conversion à un moment plutôt que de le considérer comme un processus de longue haleine associé à la construction du sens par les personnes. Nous tenions aussi à démontrer qu'il s'agit d'une expérience où les convertis n'acceptent pas, d'emblée, les discours porteurs « d'espoir religieux » auxquels ils sont exposés, mais s'activent plutôt à sélectionner les stimuli, à les choisir, et même à innover dans leur interprétation. Enfin, nous souhaitions aussi mettre en évidence l'importance de la communication intrapersonnelle et interpersonnelle dans le processus.

Afin de parvenir à rencontrer ces objectifs, nous avons adopté une démarche méthodologique qui puisse rendre compte du processus par lequel sont assimilés les objets de conscience et qui prend en considération les notions de signes et d'interprétant. Nous nous sommes donc investie, au début de notre recherche, à clarifier ces notions, afin de les repérer, par la suite, dans l'ensemble de la documentation recueillie et ayant pour objet la conversion spirituelle ou religieuse. Ensuite, nous avons lu les deux œuvres de Garaudy, soit son Testament philosophique et sa Biographie, afin de découvrir les éléments les plus démonstratifs des notions théoriques déjà étudiées. 
Notre travail consiste donc en une recherche qualitative où nous explorons le bienfondé de nos hypothèses par une analyse subjective de vérification de contenu.

Nous n'avons pas attaché d'importance à la présence de thèmes spécifiques dans le contenu, ni à la répétition de certains termes. Nous cherchions à comprendre tous les sous-entendus qui motivaient le discours de Garaudy, tout ce qu'il disait et que nous ne pouvions pas vraiment saisir à prime abord, comme sa conception philosophique de la transcendance, le contexte social dans lequel il a évolué, les mouvances idéologiques associées au remaniement des discours de l'Église catholique et du mouvement marxiste, ainsi que les aspects de l'Islam qui l'ont séduit au point de vouloir y adhérer. L'essentiel, ne se situait pas dans les données comme telles, mais plutôt dans le contenu latent, c'est-à-dire ce qui apparaît significatif pour Garaudy. Tant que nous n'avons pas eu une idée claire de la pensée de Garaudy, nous avons lu et essayer de comprendre. Nous estimons que notre travail présente des critères d'exhaustivité, de représentativité et de pertinence, dont font mention plusieurs chercheurs et avec lesquels nous pouvons aussi justifier la validité de nos propos et de nos découvertes. ${ }^{221}$

$\mathrm{Si}$, pour certains, notre démarche peut sembler aléatoire, elle nous a permis de réaliser, après un long cheminement de recherches, les constats suivants :

1) l'action de se convertir signifie de lâcher prise d'une partie des critères d'identification qui nous définissent ;

2) que les prises de décision sont conscientes, mais mues à la fois par la conscience et les autres instances psychiques ;

3) que la réflexivité dans la communication intrapersonnelle favorise la distanciation de l'esprit par rapport à ses prises de décision conscientes et inconscientes ;

4) que nos facultés télésitiques peuvent nous propulser, au-delà de nos déterminismes, dans des univers de communication que nous construisons avec notre participation volontaire ;

5) que l'ouverture à d'autres façons d'appréhender le monde correspond aussi à une habitude, initialement apprise par coerséduction, au sein des groupes d'appartenance ;

6) que, par nos échanges interpersonnels, nous nous associons à ceux qui nous ressemblent, c'est-à-dire aux personnes qui partagent un discours similaire afin de réaliser des objectifs communs ;

7) que ce discours partagé au sein des organisations, varie selon l'évolution du contexte social dans le temps et peut en venir à dissimuler des clauses inadmissibles, voire contraires, au contrat initial servant à unir les différentes parties ;

8) que la prise en compte de ces anomalies peut susciter des divergences d'opinion graves, voire des conflits éthiques pouvant générer l'exclusion du membre qui refuse de tels modifications à l'entente collective initiale ;

221 Robert Mayer et Francine Ouellet, Méthodologies de recherche pour les intervenants sociaux, Boucherville, Gaëtan Morin, 1991, p. 483-484. 
9) que la motivation à se convertir et adopter un nouveau schème d'appréhension provient également de la prise en compte de la relativité des schèmes d'appréhension. En d'autres termes, la conversion peut contribuer à reléguer au second plan l'attachement identitaire qui instaure les différences entre humains et qui permet de justifier l'imposition des significations des uns par rapport aux autres, pour favoriser la reconnaissance que tous participent, selon leur vision du monde, à décoder ce qu'ils perçoivent ;

10) que se convertir impose aussi d'endosser les termes d'un nouveau contrat en relation avec un nouveau groupe de personnes afin de réaliser, à l'intérieur d'un univers communicationnel nouveau, des projets gratifiants conformément à ses croyances.

De plus, considérant les conclusions des chercheurs de «l'École de Columbia », nous constatons :

1) que Garaudy, même s’il entreprend un débat intellectuel avec Sartre sur plus de dix ans, n'adopte pas, en fin de compte, son point de vue. Dans le doute, il accepte les conseils d'un leader d'opinion à l'intérieur de sa propre communauté de croyances ;

2) que la persuasion argumentative, lorsqu'elle touche aux croyances religieuses, ne vient pas à bout des visions du monde incommensurables ;

3) qu'il n'est pas possible de convertir l'autre si des problèmes de traduction subsistent à propos du sens particulier des termes de chaque discours, notamment la notion de transcendance ;

4) que l'ouverture à une nouvelle définition de la transcendance risque de correspondre à la perception des représentations acquises initialement auprès des personnes ressources du milieu familial qui inspirent le plus confiance sur un sujet donné (l'attrait identitaire exercé par la grand-mère de Garaudy, à la fois catholique et dévote et d'origine maure) ;

5) que les personnes qui circulent dans des communautés d'interprétation différentes, et qui ont donc l'habitude de la réception active, risquent plus facilement de percevoir les contradictions relatives aux discours officiels des organisations ;

6) que la conversion, si elle sert à résoudre des conflits liés à la perception de telles anomalies, est également motivée par la prise en compte de bénéfices notables associés à l'amélioration du statut social ou encore à la possibilité de recouvrer, relativement à cet état, des avantages perdus (en particulier, la reconnaissance).

Évidemment, nous estimons que, pour véritablement apprécier ces conclusions, il faudrait offrir d'autres analyses exhaustives du processus de conversion religieuse à partir de récits autobiographiques rendant compte des changements de la vision du monde du converti. En procédant de la sorte, il serait alors peut-être possible de percevoir, dans chacun des cas, quelles interactions semblent contribuer à la construction de l'expérience de conversion et ainsi établir la récurrence de certaines variables aux dépens d'autres facteurs. Aussi, pour nous, l'étude de la conversion religieuse ne présente pas, pour le moment, un profil défini de données à vérifier pour améliorer la 
compréhension du phénomène. Si, à notre avis, la recherche sur cet objet d'études semble plus prometteuse lorsque l'analyse du phénomène est effectuée sur une longue période de temps de la vie du converti, nous réalisons aussi les complications que ceci comporte sur le plan méthodologique.

Ainsi, il est impossible de prédire qui, éventuellement, se convertira afin de l'observer et le questionner avant, pendant et après cette expérience. De plus, les entrevues orales effectuées à la suite du rituel, ne semblent rendre compte de l'expérience que très partiellement, parce que l'interprétation du converti reste alors très souvent collée à son vécu immédiat. ${ }^{222}$ L'acte de relater, par écrit, l'expérience de conversion dans le contexte d'une vie, semble plus propice à créer un espace de distanciation avec soi, qui permet de rendre compte de l'élaboration du sens de cette expérience pour le converti. Aussi, cela ne nous laisse-t-il comme alternative que de recourir aux documents biographiques qui relatent ce type d'expérience, mais qui sont aussi offerts, malheureusement, dans une disponibilité restreinte. Sans parler du fait que nous ne pouvons que travailler sur les informations qui nous sont données dans ces manuscrits ; à moins de compléter l'analyse par les commentaires des convertis s'ils sont toujours vivants et acceptent de répondre à une telle proposition. Il serait peut-être possible de remédier en partie à ces contraintes en analysant des conversions autres que religieuses comme des changements d'allégeance politique ou de paradigmes scientifiques. Bien des éventualités restent à être explorées afin de rendre compte avec plus de subtilité de la complexité de la construction du phénomène de conversion du point de vue communicationnel.

222 À moins que le converti tienne un journal de bord très exhaustif. 


\section{Bibliographie}

Retour à la table des matières

Allievi, Stefano, 1998. Les convertis à l'islam : Les nouveaux musulmans d'Europe. Paris : L'Harmattan.

Assouline, Pierre. 1992. Les nouveaux convertis : Enquête sur des chrétiens, des juifs et des musulmans pas comme les autres. Coll. « Folio/Actuel ». Paris : Gallimard, p. 201-292.

Attalah, Paul. 1989. « Introduction générale ». In Théories de la communication, histoire, contexte, pouvoir. Sillery (Qué.) : Télé-université, Presses de l'université du Québec, p.1-38.

Augé, Marc. 1994. Le sens des autres : Actualité de l'anthropologie. Paris : Fayard, p. 9-87.

Bastide, Georges. 1966. La conversion spirituelle, deuxième édition. Paris : PUF.

Bayart, Jean-François. 1996. «La culture un mot à jeter?». In L'illusion identitaire. Paris : Fayard, p. 69-125.

Berger, Peter et Thomas Luckmann. 1996. La construction sociale de la réalité, deuxième édition. Paris : Meridiens Klincksieck.

Billette, André. 1975. Récits et réalités d'une conversion. Montréal: Presses de l'Université de Montréal. 
Bougnoux, Daniel. 2001. Introduction aux sciences de la communication, nouvelle édition. Paris : La découverte, p. 18-82.

Bourdieu, Pierre et Loïc J. D. Wacquant. 1992. «Habitus, Illusio et rationalité ». In Réponses : Pour une anthropologie réflexive. Paris : Seuil, p. 91-114.

Bourdieu, Pierre. 1994. Raisons pratiques : Sur la théorie de l'action. Paris : Seuil, p. 39-57 et 101-217.

Carey, James W. 1988. « Reconceiving « Mass » and « Media » ». In Communication as Culture : Essays on Media and Society. New York, London (Great Britain) : Routledge, p. 69-88.

Carey, James W. 1997. «“A Republic, If You Can Keep It”: Liberty and Public Life in the Age of Glasnost ». In James Carey: A Critical Reader. Eve Stryker Munson and Catherine A. Warren, editors. Minneapolis: University of Minnesota Press, p. 207-227.

Casanova, Antoine. 1985. Le concile vingt ans après. Paris : Messidor, p. 15-111.

Dumont, Fernand. 1974. Les idéologies. Paris : PUF, p. 5-40.

Durrand, J.-P. et F.-X. Merrien. 1991. Sortie de siècle : La France en mutation. Coll. « Essentiel ». Paris : Vigot, p. 96-260.

Enriquez, Eugène. 1997. "Les enjeux éthiques des organisations ». In Les jeux du pouvoir et du désir dans l'entreprise. Paris : Desclée de Brouwer, p. 115137.

Ferry, Luc. L’Homme-Dieu ou le Sens de la vie. 1996. Paris, Grasset.

Giannini, Humberto. 1996. «Accueillir l'étrangeté ». In La tolérance : Pour un humanisme hérétique, dir. publ. Claude Sahel. Paris, Autrement, p. 16-30.

Giddens, Anthony. 1994. Les conséquences de la modernité. Paris : L’Harmattan, p. 11-60.

Grand'Maison, Jacques. 1999. Quand le jugement fout le camp : Essai sur la déculturation. Montréal : Fides.

Guillebaud, Jean-Claude. 1999. La refondation du monde. Paris : Seuil, p. 9-156 et p. 279-320.

Huntington, Samuel P. 2000. Le choc des civilisations. Paris : Odile Jacob, p. 81-172. 
James, William. 1999. «La conversion ». In L'expérience religieuse : Essai de psychologie descriptive. Paris : Bibliothèque de l'Homme, p. 223-292.

Katz Elihu. 1973. «Les deux étages de la communication (The two-step flow of communication). In Sociologie de l'information, dir. publ. F. Balle et J. G. Padioleau. Paris : Larousse, p. 285-303.

Kepel, Gilles. 1994. À l'ouest d'Allah. Paris : Seuil, p. 11-100 et p. 223-358.

Kuhn, Thomas S. 1983. La structure des révolutions scientifiques. Paris : Flammarion.

Laing, R. D. «The Spiral of Reciprocal Perspectives » In Interpersonal Perception : A Theory and a Method of Research, New York : Harper, p. 30-45.

Laplantine, François. 1996. La description ethnographique. Paris : Nathan, p. 18.

Laplantine, François. 1999. Je, nous et les autres : Être humain au-delà des appartenances. Paris : Le Pommier.

Linton, Ralph. 1977. Les fondements culturels de la personnalité. Paris : Dunod.

Maalouf, Amin. 1998. Les Identités meurtrières. Paris: Grasset.

Morin, Edgar. Pour sortir du vingtième siècle. Paris : Fernand Nathan, 1981, p. 7-38.

Morin, Edgar. 1982. Science avec conscience. Paris : Fayard, p. 7-25 et p. 295-313.

Moscovici, Serge. 1981. «L'origine des attachements affectifs dans la société » et «Éros et Mimésis ». In L'âge des foules, nouv. éd. ent. ref. Bruxelles : Complexe, p. 335-353 et p. 354-369.

Moscovici, Serge. 1988. La machine à faire des Dieux : Sociologie et psychologie. Paris : Fayard.

Noël, Guy.1997. Le développement international et la gestion de projet. Sainte-Foy (Qué.) : Presses de l'Université du Québec.

Peach, Wesley. 2001. Itinéraires de conversion. Montréal : Fides.

Rambo, Lewis R. 1993. Understanding Religious Conversion. New Haven and London: Yale University Press. 
Ravault, René-Jean. 1993. «La communication dans le monde : un rêve américain irréalisable même aux États-Unis ». In T. 1 du Dictionnaire critique de la communication, dir. publ. Lucien Sfez. Paris : PUF, p. 72-86.

Ravault, René-Jean. 1996. «Développement durable, communication et réception active ». In Communication et développement international, dir. publ. Thérèse Paquet-Sévigny. Sainte-Foy (Qué.) : Presses de l'Université du Québec, p. 59-79.

Revel, Jean-François et Matthieu Ricard. 1999. Le moine et le philosophe, édition revue et corrigée. Paris : Nil.

Rocher, Lisbeth et Fatima Cherqaoui. 1986. D'une foi l'autre : Les conversions à l'islam en Occident. Paris : Seuil.

Rosen, Jay. 1997. «Introduction / «We'll Have That Conversation » : Journalism and Democracy in the Thought of James W. Carey ». In James Carey : A Critical Reader. Eve Stryker Munson and Catherine A. Warren, editors. Minneapolis : University of Minnesota Press, p. 191-206.

Sfez, Lucien. 1984. «La théorie politique du surcode » et «Une décision libératrice ». In La décision. Paris : PUF, p.110-124 et 125-127.

Stoetzel, Jean. 1963. «La socialisation de l'individu» et «La perception». In La psychologie sociale. Paris : Flammarion, p. 58-84 et p. 94-110.

Strauss, Anselm L. 1992. Comp. Isabelle Baszanger. La trame de la négociation: Sociologie qualitative et interactionnisme. Paris : L'Harmattan, p. 7-21.

Thayer, Lee. 1987. On Communication : Essays in Understanding. Norwood (New Jersey) : Ablex, p. vii-144.

Thayer, Lee. 1997. «Culture and Communicability ». In Pieces : Toward a Revisioning of Communication/Life. Greenwich (Connecticut) et London (England) : Ablex, p. 4-17

Todorov, Tzvetan. 1982. La conquête de l'Amérique : La question de l'autre. Paris : Seuil.

Todorov, Tzvetan. 1991. Les morales de l'histoire, Paris, Hachette, p.51-98.

Todorov, Tzvetan. 2000. Mémoire du mal, tentation du bien : Enquête sur le siècle. Paris : Robert Laffont. 
Touraine, Alain. 1984. «La vie sociale a-t-elle un centre?». In Le retour de l'acteur : Essai de sociologie. Paris : Fayard, p.91-104.

W. Riley, John et Matilda W. Riley. 1973. «La communicationde masse et le système social ». In Sociologie de l'information, dir. publ. F. Balle et J. G. Padioleau. Paris : Larousse, p. 69-96.

Zeldin, Theodore. 1994. «How humans become hospitable to each other ». In An Intimate History of Humanity. New York : Harper Collins, p. 426-464.

\section{Euvres de Roger Garaudy}

Garaudy Roger. 1985. Biographie du XXe siècle : Le testament philosophique de Roger Garaudy. Paris : Tougui.

_- $\quad$ 1989. Mon tour du siècle en solitaire : Mémoires. Paris : Robert Laffont.

\section{Actes de colloque}

Mac Cana, Proinsias (éd.), Michel Meslin (éd.), Collège des Irlandais (Paris), Royal Irish Academy. 1986. Rencontres de religions: Actes du colloque du Collège des Irlandais / sous les auspices de l'Académie royale irlandaise (juin 1981). Paris : Les belles lettres.

\section{Articles de revues et de périodiques}

Allevi, Stefano. «Pour une sociologie des conversions : lorsque des Européens deviennent musulmans ». Social Compass, 46(3), 1999, p. 283-300.

Billette, André. «Se raconter une histoire.. : Pour une analyse révisée de la conversion ». Social Compass, XXIII, 1976/1, p. 47-56.

Daynes, Sarah. "Processus de conversion et modesd'identification à l'islam : l'exemple de la France et des États-Unis ». Social Compass, 46(3), 1999, p. 313-323. 
García-Arenal, Mercedes. «Les conversions d'Européens à l'islam dans l'histoire : esquisse générale ». Social Compass, 46(3), 1999, p. 273-281.

Hall, Stuart. « Codage/Décodage ». Réseaux, N68, CNET, 1994, p. 29-39.

Köse, Ali. « The Journey from the Secular to the Sacred : Experiences of Native British Converts to Islam ». Social Compass, 46(3), 1999, p. 301-312.

Leland, John. « Searching for a Holy Spirit ». Newsweek, May 8, 2000, p. 61-63.

Lofland, John et Norman Skonovd. «Conversion Motifs ». Journal for the Scientific Study of Religion, 20(4), 1981, p. 373-385.

Luckmann, Thomas. «The Religious Situation in Europe: the Background to Contemporary Conversions ». Social Compass, 46(3), 1999, p. 251-258.

Sultán, Madeleine. «Choosing Islam : a Study of Swedish Converts ». Social Compass, 46(3), 1999, p. 325-335.

Taylor Brian. «Conversion and Cognition : An Area for Empirical Study in the Microsociology of Religious Knowledge ». Social Compass, XXIII, 1976/1, p. $5-22$.

Tehranian, Majid. «La malédiction de la modernité : dialectique de la modernisation et de la communication ». Revue internationale de sciences sociales, Vol. XXXII, 1980, $\mathrm{N}^{\circ} 2$, p.263-280.

Wohlrab-Sahr, Monika. «Conversion to Islam : Between Syncretism and Symbolic Battle ». Social Compass, 46(3), 1999, p. 351-362.

\section{Précis de rédaction}

Beaud, Michel et Daniel Latouche. 1988. L'art de la thèse : Comment préparer et rédiger une thèse, un mémoire ou tout autre travail universitaire. Montréal : Boréal.

Bouthat, Chantal. 1993. Guide de présentation des mémoires et thèses. Montréal : Université du Québec à Montréal.

Mayer, Robert et Francine Ouellet. 1991. «L'analyse de contenu ». In Méthodologies de recherche pour les intervenants sociaux. Boucherville : Gaëtan Morin, p. 474-499. 


\section{Dictionnaires}

Robert, Paul. 1990. Le petit Robert 1 : Dictionnaire de la langue française, nouvelle édition revue et corrigée. Rédaction dirigée par A.Rey et J. Rey-Debove. Paris : Le Robert.

Robert, Paul (dir. de publ.). Le Petit Robert 2 : Dictionnaire universel des noms propres alphabétique et analogique, nouvelle édition revue et corrigée. Rédaction dirigée par Alain Rey. Paris : Le Robert.

\section{Adresses de sites Internet}

http://www.republique-des-lettres.com

http://www.adl.org

http://www.codoh.com.

Fin du texte 